\title{
Synthesis of Polysubstituted Pyrroles via Silver-Catalyzed Oxidative Radical Addition of Cyclopropanols to Imines
}

Yulu Zhou, ${ }^{\dagger}$ Mingchang Wu, ${ }^{\dagger}$ Yi Liu, Cungui Cheng, and Gangguo Zhu ${ }^{*}$ Key Laboratory of the Ministry of Education for Advanced Catalysis Materials, Department of Chemistry, Zhejiang Normal University, 688 Yingbin Road, Jinhua 321004, P. R. China E-mail: gangguo@zjnu.cn

Table of Contents

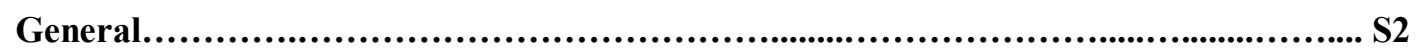

General Procedure for Experiments and Analytical data.....................................S2-S14

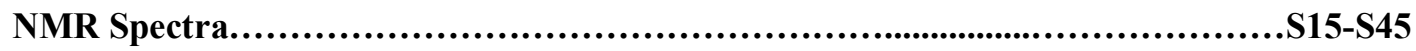


General. Melting points reported here were measured by a melting point instrument and were uncorrected. ${ }^{1} \mathrm{H},{ }^{13} \mathrm{C}$, and ${ }^{19} \mathrm{~F}$ NMR spectra were measured on a 600 or $400 \mathrm{MHz}$ NMR spectrometers with $\mathrm{CDCl}_{3}$ as the solvent with tetramethylsilane (TMS) as the internal standard. The following abbreviations were used to explain the multiplicities: $\mathrm{s}=$ singlet, $\mathrm{d}=$ doublet, $\mathrm{t}=$ triplet, $\mathrm{q}=$ quartet, $\mathrm{m}=$ multiplet. High-resolution mass spectra (HRMS) analyses were run with a TOF MS instrument using an ESI source. The products were purified by column chromatography using silica gel (300-400 mesh) with a mixture of petroleum ether and EtOAc as the eluent. Imines $\mathbf{1}^{1}$ and cyclopropanols $\mathbf{2}^{2}$ are prepared according to previously reported literature.

\section{General Procedure for the Silver-Catalyzed Oxidative Radical Addition of Cyclopropanols to Imines}

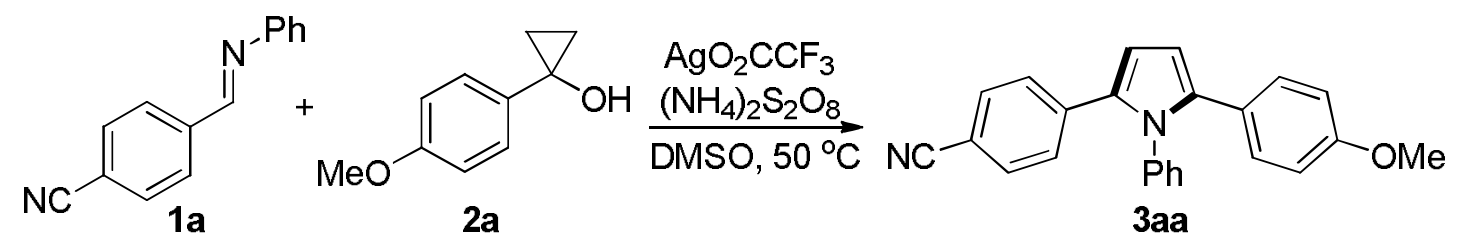

To a solution of 1a $(51.5 \mathrm{mg}, 0.25 \mathrm{mmol})$ and $2 \mathrm{a}(82.0 \mathrm{mg}, 0.5 \mathrm{mmol})$ in $6 \mathrm{~mL}$ of dry DMSO was added $\mathrm{AgO}_{2} \mathrm{CCF}_{3}(5.5 \mathrm{mg}, 0.025 \mathrm{mmol})$ and $\left(\mathrm{NH}_{4}\right)_{2} \mathrm{~S}_{2} \mathrm{O}_{8}(171.0 \mathrm{mg}, 0.75 \mathrm{mmol})$ under a nitrogen atmosphere. After stirring at $50{ }^{\circ} \mathrm{C}$ for 10 hours, the reaction mixture was quenched by water, extracted by EtOAc, dried by anhydrous $\mathrm{Na}_{2} \mathrm{SO}_{4}$, and concentrated. Column chromatography on silica gel (petroleum ethers/EtOAc $=50: 1)$ gave $71 \mathrm{mg}(81 \%$ yield $)$ of 3aa as a yellow solid; $\mathrm{mp}$ 207-209 ${ }^{\circ} \mathrm{C} ;{ }^{1} \mathrm{H}$ NMR $\left(600 \mathrm{MHz}, \mathrm{CDCl}_{3}\right) \delta 7.41(\mathrm{~d}, J=8.3 \mathrm{~Hz}, 2 \mathrm{H}), 7.33-7.26(\mathrm{~m}, 3 \mathrm{H}), 7.10(\mathrm{~d}, J$ $=8.0 \mathrm{~Hz}, 2 \mathrm{H}), 7.03(\mathrm{~d}, J=7.5 \mathrm{~Hz}, 2 \mathrm{H}), 6.97(\mathrm{~d}, J=7.8 \mathrm{~Hz}, 2 \mathrm{H}), 6.73(\mathrm{~d}, J=8.5 \mathrm{~Hz}, 2 \mathrm{H}), 6.59$ (d, $J=3.1 \mathrm{~Hz}, 1 \mathrm{H}), 6.43(\mathrm{~d}, J=3.4 \mathrm{~Hz}, 1 \mathrm{H}), 3.75(\mathrm{~s}, 3 \mathrm{H}) ;{ }^{13} \mathrm{C} \mathrm{NMR}\left(151 \mathrm{MHz}, \mathrm{CDCl}_{3}\right) \delta 158.5$, 138.5, 137.7, 137.6, 133.0, 131.7, 130.1, 129.1, 128.7, 128.1, 127.7, 125.1, 119.1, 113.4, 111.8, 109.9, 108.8, 55.1; HRMS (ESI) $m / z$ : $[\mathrm{M}+\mathrm{Na}]^{+}$Calcd for $\mathrm{C}_{24} \mathrm{H}_{18} \mathrm{~N}_{2} \mathrm{ONa}$ 373.1311; Found 373.1310.

\footnotetext{
${ }^{1}$ Leitch, J.; Rogova, T.; Duarte, F.; Dixion, D. Angew. Chem. Int. Ed. 2020, 59, 4121.

${ }^{2}$ Zhan, J.; Wu, M.; Wei, D.; Wei, B.; Han, B. ACS Catal. 2019, 9, 4179.
} 


\section{Scale-Up Synthesis of 3aa.}

To a solution of 1a (206 mg, $1.0 \mathrm{mmol})$ and $\mathbf{2 a}(328 \mathrm{mg}, 2.0 \mathrm{mmol})$ in $20 \mathrm{~mL}$ of dry DMSO was added $\mathrm{AgO}_{2} \mathrm{CCF}_{3}(22 \mathrm{mg}, 0.1 \mathrm{mmol})$ and $\left(\mathrm{NH}_{4}\right)_{2} \mathrm{~S}_{2} \mathrm{O}_{8}(684 \mathrm{mg}, 3.0 \mathrm{mmol})$ under a nitrogen atmosphere. After stirring at $50{ }^{\circ} \mathrm{C}$ for 15 hours, the reaction mixture was quenched by water, extracted by EtOAc, dried by anhydrous $\mathrm{Na}_{2} \mathrm{SO}_{4}$, and concentrated. Column chromatography on silica gel (petroleum ethers/EtOAc $=50: 1)$ gave $266 \mathrm{mg}(76 \%$ yield) of 3aa as a yellow solid.<smiles>COc1ccc(-c2ccc(-c3ccc(C#N)cc3)n2-c2ccc(C)cc2)cc1</smiles>

Compound 3ba: $76 \mathrm{mg}, 84 \%$ yield, white solid; $\mathrm{mp} 210-212{ }^{\circ} \mathrm{C}$; Flash column chromatography conditions: petroleum ethers/EtOAc $=50: 1 ;{ }^{1} \mathrm{H}$ NMR $\left(600 \mathrm{MHz}, \mathrm{CDCl}_{3}\right) \delta 7.42(\mathrm{~d}, J=8.5 \mathrm{~Hz}$, 2H), $7.11(\mathrm{~d}, J=8.5 \mathrm{~Hz}, 2 \mathrm{H}), 7.08(\mathrm{~d}, J=8.1 \mathrm{~Hz}, 2 \mathrm{H}), 6.98(\mathrm{~d}, J=8.8 \mathrm{~Hz}, 2 \mathrm{H}), 6.91(\mathrm{~d}, J=8.2$ Hz, 2H), 6.73 (d, $J=8.8 \mathrm{~Hz}, 2 \mathrm{H}), 6.58(\mathrm{~d}, J=3.7 \mathrm{~Hz}, 1 \mathrm{H}), 6.41$ (d, $J=3.7 \mathrm{~Hz}, 1 \mathrm{H}), 3.76(\mathrm{~s}, 3 \mathrm{H})$, 2.36 (s, 3H); ${ }^{13} \mathrm{C}$ NMR $\left(151 \mathrm{MHz}, \mathrm{CDCl}_{3}\right) \delta 158.4,137.7,137.7,137.7,135.9,133.0,131.7,130.1$, 129.7, 128.4, 128.1, 125.3, 119.2, 113.4, 111.7, 109.7, 108.7, 55.1, 21.2; HRMS (ESI) $m / z:[\mathrm{M}+$ $\mathrm{Na}]^{+}$Calcd for $\mathrm{C}_{25} \mathrm{H}_{20} \mathrm{~N}_{2} \mathrm{ONa}$ 387.1468; Found 387.1469.<smiles>COc1ccc2c(c1)c1ccccc1n1c(-c3ccc(C#N)cc3)ccc21</smiles>

$3 \mathrm{ca}$

Compound 3ca: $76 \mathrm{mg}, 83 \%$ yield, white solid; $\mathrm{mp} 215-217{ }^{\circ} \mathrm{C}$; Flash column chromatography conditions: petroleum ethers/EtOAc $=50: 1 ;{ }^{1} \mathrm{H}$ NMR $\left(600 \mathrm{MHz}, \mathrm{CDCl}_{3}\right) \delta 7.39(\mathrm{~d}, J=8.5 \mathrm{~Hz}$, 2H), 7.28-7.26 (m, 1H), 7.20-7.14 (m, 3H), 7.09 (d, $J=8.5 \mathrm{~Hz}, 2 \mathrm{H}), 7.00-6.96$ (m, 2H), 6.70 (d, $J=8.8 \mathrm{~Hz}, 2 \mathrm{H}), 6.64(\mathrm{~d}, J=3.8 \mathrm{~Hz}, 1 \mathrm{H}), 6.47(\mathrm{~d}, J=3.8 \mathrm{~Hz}, 1 \mathrm{H}), 3.74(\mathrm{~s}, 3 \mathrm{H}), 1.78(\mathrm{~s}, 3 \mathrm{H}) ;{ }^{13} \mathrm{C}$ NMR (151 MHz, $\left.\mathrm{CDCl}_{3}\right) \delta 158.5,137.9,137.7,137.6,136.5,133.0,131.8,131.1,129.7,129.4$, 
$128.7,127.2,126.7,125.2,119.1,113.5,111.5,109.5,108.7,55.1,17.5$; HRMS (ESI) $m / z:[\mathrm{M}+$ $\mathrm{Na}]^{+}$Calcd for $\mathrm{C}_{25} \mathrm{H}_{20} \mathrm{~N}_{2} \mathrm{ONa}$ 387.1468; Found 387.1460.<smiles>COc1ccc(-c2ccc(-c3ccc(C#N)cc3)n2-c2ccc(F)cc2)cc1</smiles>

3da

Compound 3da: $73 \mathrm{mg}, 80 \%$ yield, white solid; mp $167-169{ }^{\circ} \mathrm{C}$; Flash column chromatography conditions: petroleum ethers/EtOAc $=50: 1 ;{ }^{1} \mathrm{H}$ NMR $\left(400 \mathrm{MHz}, \mathrm{CDCl}_{3}\right) \delta 7.46(\mathrm{~d}, J=8.4 \mathrm{~Hz}$, 2H), $7.41(\mathrm{~d}, J=8.6 \mathrm{~Hz}, 2 \mathrm{H}), 7.10(\mathrm{~d}, J=8.4 \mathrm{~Hz}, 2 \mathrm{H}), 6.96(\mathrm{~d}, J=8.7 \mathrm{~Hz}, 2 \mathrm{H}), 6.89(\mathrm{~d}, J=8.6$ $\mathrm{Hz}, 2 \mathrm{H}), 6.76(\mathrm{~d}, J=8.7 \mathrm{~Hz}, 2 \mathrm{H}), 6.58(\mathrm{~d}, J=3.7 \mathrm{~Hz}, 1 \mathrm{H}), 6.41(\mathrm{~d}, J=3.7 \mathrm{~Hz}, 1 \mathrm{H}), 3.78(\mathrm{~s}, 3 \mathrm{H})$; ${ }^{13} \mathrm{C} \mathrm{NMR}\left(151 \mathrm{MHz}, \mathrm{CDCl}_{3}\right) \delta 161.7(\mathrm{~d}, J=248.9 \mathrm{~Hz}), 158.6,137.8,137.4,134.5(\mathrm{~d}, J=3.1 \mathrm{~Hz})$, 133.0, 131.8, 130.3 (d, $J=8.4 \mathrm{~Hz}), 130.2,128.2,124.9,119.0,116.2(\mathrm{~d}, J=22.6 \mathrm{~Hz}), 113.5$, 111.9, 109.9, 109.1, 55.2; ${ }^{19} \mathrm{~F}$ NMR (565 MHz, $\left.\mathrm{CDCl}_{3}\right) \delta-113.0$; HRMS (ESI) $m / z:[\mathrm{M}+\mathrm{Na}]^{+}$ Calcd for $\mathrm{C}_{24} \mathrm{H}_{17} \mathrm{FN}_{2} \mathrm{ONa}$ 391.1217; Found 391.1219.<smiles>COc1ccc(-c2ccc(-c3ccc(C#N)cc3)n2-c2ccc(Cl)cc2)cc1</smiles>

Compound 3ea: $73 \mathrm{mg}, 77 \%$ yield, white solid; mp $187-190{ }^{\circ} \mathrm{C}$; Flash column chromatography conditions: petroleum ethers/EtOAc $=50: 1 ;{ }^{1} \mathrm{H}$ NMR $\left(600 \mathrm{MHz}, \mathrm{CDCl}_{3}\right) \delta 7.46(\mathrm{~d}, J=8.3 \mathrm{~Hz}$, 2H), 7.28-7.24 (m, 2H), $7.10(\mathrm{~d}, J=8.3 \mathrm{~Hz}, 2 \mathrm{H}), 6.96(\mathrm{t}, J=8.6 \mathrm{~Hz}, 4 \mathrm{H}), 6.76(\mathrm{~d}, J=8.7 \mathrm{~Hz}, 2 \mathrm{H})$, $6.58(\mathrm{~d}, J=3.7 \mathrm{~Hz}, 1 \mathrm{H}), 6.42(\mathrm{~d}, J=3.7 \mathrm{~Hz}, 1 \mathrm{H}), 3.78(\mathrm{~s}, 3 \mathrm{H}) ;{ }^{13} \mathrm{C} \mathrm{NMR}\left(151 \mathrm{MHz}, \mathrm{CDCl}_{3}\right) \delta$ 158.7, 137.6, 137.3, 137.0, 133.5, 132.9, 131.9, 130.2, 129.9, 129.3, 128.2, 124.8, 119.0, 113.6, 112.1, 110.2, 109.2, 55.2; HRMS (ESI) $m / z$ : $[\mathrm{M}+\mathrm{Na}]^{+}$Calcd for $\mathrm{C}_{24} \mathrm{H}_{17} \mathrm{ClN}_{2} \mathrm{ONa}$ 407.0922; Found 407.0928. 
<smiles>COc1ccc(-c2ccc(-c3ccc(C#N)cc3)n2-c2ccc(Br)cc2)cc1</smiles>

Compound $3 \mathrm{fa}$ : $83 \mathrm{mg}$, 78\% yield, white solid; $\mathrm{mp} 185-187{ }^{\circ} \mathrm{C}$; Flash column chromatography conditions: petroleum ethers/EtOAc $=50: 1 ;{ }^{1} \mathrm{H}$ NMR $\left(600 \mathrm{MHz}, \mathrm{CDCl}_{3}\right) \delta 7.49(\mathrm{~d}, J=8.4 \mathrm{~Hz}$, 2H), 7.44 (s, 2H), 7.13 (d, $J=8.4 \mathrm{~Hz}, 2 \mathrm{H}), 6.99$ (d, $J=8.7 \mathrm{~Hz}, 2 \mathrm{H}), 6.91$ (d, $J=8.6 \mathrm{~Hz}, 2 \mathrm{H}), 6.79$ $(\mathrm{d}, J=8.8 \mathrm{~Hz}, 2 \mathrm{H}), 6.60(\mathrm{~d}, J=3.7 \mathrm{~Hz}, 1 \mathrm{H}), 6.44(\mathrm{~d}, J=3.7 \mathrm{~Hz}, 1 \mathrm{H}), 3.81(\mathrm{~s}, 3 \mathrm{H}),{ }^{13} \mathrm{C}$ NMR $(151$ $\left.\mathrm{MHz}, \mathrm{CDCl}_{3}\right) \delta 158.7,137.6,137.5,137.3,132.9,132.3,131.9,130.2,130.2,128.3,124.8,121.5$, 119.0, 113.6, 112.2, 110.2, 109.2, 55.2; HRMS (ESI) $m / z$ : $[\mathrm{M}+\mathrm{Na}]^{+}$Calcd for $\mathrm{C}_{24} \mathrm{H}_{17} \mathrm{BrN}_{2} \mathrm{ONa}$ 451.0416; Found 451.0406.<smiles>COc1ccc(-c2ccc(-c3ccc(C#N)cc3)n2-c2ccc(I)cc2)cc1</smiles>

3ga

Compound 3ga: $81 \mathrm{mg}$, $68 \%$ yield, white solid; mp $157-160{ }^{\circ} \mathrm{C}$; Flash column chromatography conditions: petroleum ethers/EtOAc $=50: 1 ;{ }^{1} \mathrm{H}$ NMR $\left(600 \mathrm{MHz}, \mathrm{CDCl}_{3}\right) \delta 7.60(\mathrm{~d}, J=8.5 \mathrm{~Hz}$, 2H), $7.46(\mathrm{~d}, J=8.4 \mathrm{~Hz}, 2 \mathrm{H}), 7.10(\mathrm{~d}, J=8.4 \mathrm{~Hz}, 2 \mathrm{H}), 6.98-6.94(\mathrm{~m}, 2 \mathrm{H}), 6.81-6.72(\mathrm{~m}, 4 \mathrm{H})$, $6.57(\mathrm{~d}, J=3.7 \mathrm{~Hz}, 1 \mathrm{H}), 6.41(\mathrm{~d}, J=3.7 \mathrm{~Hz}, 1 \mathrm{H}), 3.78(\mathrm{~s}, 3 \mathrm{H}) ;{ }^{13} \mathrm{C}$ NMR $\left(151 \mathrm{MHz}, \mathrm{CDCl}_{3}\right) \delta$ 158.7, 138.3, 138.2, 137.5, 137.3, 132.8, 131.9, 130.4 130.2, 128.3, 124.8, 113.6, 112.2, 110.3, 109.2, 99.9, 92.9, 55.2; HRMS (ESI) $m / z$ : $[\mathrm{M}+\mathrm{Na}]^{+}$Calcd for $\mathrm{C}_{24} \mathrm{H}_{17} \mathrm{IN}_{2} \mathrm{ONa}$ 499.0278; Found 499.0262 . 


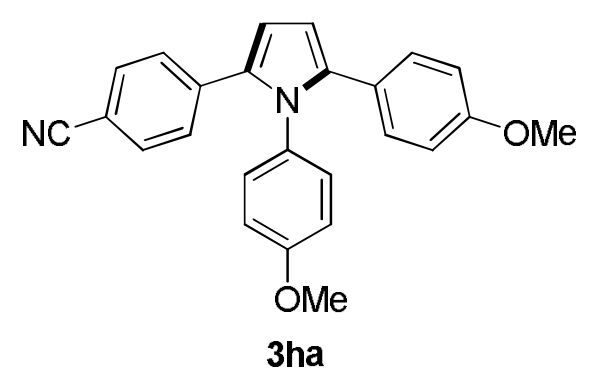

Compound 3ha: $79 \mathrm{mg}$, 83\% yield, white solid; mp 223-235 ${ }^{\circ} \mathrm{C}$; Flash column chromatography conditions: petroleum ethers/EtOAc $=30: 1 ;{ }^{1} \mathrm{H} \mathrm{NMR}\left(600 \mathrm{MHz}, \mathrm{CDCl}_{3}\right) \delta 7.46(\mathrm{~d}, J=8.4 \mathrm{~Hz}, 2 \mathrm{H})$, $7.15(\mathrm{~d}, J=8.4 \mathrm{~Hz}, 2 \mathrm{H}), 7.02(\mathrm{~d}, J=8.7 \mathrm{~Hz}, 2 \mathrm{H}), 6.98$ (d, $J=8.8 \mathrm{~Hz}, 2 \mathrm{H}), 6.83$ (d, $J=8.8 \mathrm{~Hz}$, 2H), $6.77(\mathrm{~d}, J=8.7 \mathrm{~Hz}, 2 \mathrm{H}), 6.60(\mathrm{~d}, J=3.7 \mathrm{~Hz}, 1 \mathrm{H}), 6.44(\mathrm{~d}, J=3.7 \mathrm{~Hz}, 1 \mathrm{H}), 3.84(\mathrm{~s}, 3 \mathrm{H}), 3.79$ (s, 3H); ${ }^{13} \mathrm{C}$ NMR $\left(151 \mathrm{MHz}, \mathrm{CDCl}_{3}\right) \delta 158.8,158.5,139.4,137.7,133.1,131.7,131.3,130.1$, $129.7,128.1,125.3,119.2,114.3,113.5,111.5,109.6,108.8,55.4,55.2$; HRMS (ESI) $m / z:[\mathrm{M}+$ $\mathrm{H}]^{+}$Calcd for $\mathrm{C}_{25} \mathrm{H}_{21} \mathrm{~N}_{2} \mathrm{O}_{2} 381.1598$; Found 381.1596.<smiles>COc1ccc(-c2ccc(-c3ccc(C#N)cc3)n2-c2ccc(C(F)(F)F)cc2)cc1</smiles>

3ia

Compound 3ia: $38 \mathrm{mg}$, 37\% yield, yellow solid; mp $227-230{ }^{\circ} \mathrm{C}$; Flash column chromatography conditions: petroleum ethers/EtOAc $=50: 1 ;{ }^{1} \mathrm{H}$ NMR $\left(600 \mathrm{MHz}, \mathrm{CDCl}_{3}\right) \delta 7.54(\mathrm{~d}, J=8.3 \mathrm{~Hz}, 2 \mathrm{H})$, 7.49-7.44 (m, 2H), 7.12 (d, J=8.2 Hz, 2H), 7.10-7.06 (m, 2H), 6.96-6.92 (m, 2H), 6.78-6.74 (m, 2H), $6.59(\mathrm{~d}, J=3.7 \mathrm{~Hz}, 1 \mathrm{H}), 6.44(\mathrm{~d}, J=3.7 \mathrm{~Hz}, 1 \mathrm{H}), 3.78(\mathrm{~s}, 3 \mathrm{H}) ;{ }^{13} \mathrm{C} \mathrm{NMR}\left(151 \mathrm{MHz}, \mathrm{CDCl}_{3}\right)$ $\delta 158.8,141.5,137.6,137.2,132.9,131.9,130.2,129.6(\mathrm{q}, J=32.8 \mathrm{~Hz}), 128.9,128.3,126.2(\mathrm{q}, J$ $=3.7 \mathrm{~Hz}), 124.6,123.6(\mathrm{q}, J=272.5 \mathrm{~Hz}), 118.8,113.6,112.5,110.6,109.4,55.2 ;{ }^{19} \mathrm{~F}$ NMR $(565$ $\mathrm{MHz}, \mathrm{CDCl}_{3}$ ) $\delta$-62.4; HRMS (ESI) $m / z$ : $[\mathrm{M}+\mathrm{H}]^{+}$Calcd for $\mathrm{C}_{25} \mathrm{H}_{18} \mathrm{~F}_{3} \mathrm{~N}_{2} \mathrm{O}$ 419.1366; Found 419.1363.<smiles>CCCCCCCn1c(-c2ccc(C#N)cc2)ccc1-c1ccc(OC)cc1</smiles> 
Compound 3ja: $19 \mathrm{mg}, 22 \%$ yield, white solid; mp 185-189 ${ }^{\circ} \mathrm{C}$; Flash column chromatography conditions: petroleum ethers/EtOAc $=50: 1 ;{ }^{1} \mathrm{H} \mathrm{NMR}\left(600 \mathrm{MHz}, \mathrm{CDCl}_{3}\right) \delta 7.69(\mathrm{~d}, J=8.3 \mathrm{~Hz}, 2 \mathrm{H})$, $7.55(\mathrm{~d}, J=8.3 \mathrm{~Hz}, 2 \mathrm{H}), 7.36(\mathrm{~d}, J=8.7 \mathrm{~Hz}, 2 \mathrm{H}), 6.98(\mathrm{~d}, J=8.7 \mathrm{~Hz}, 2 \mathrm{H}), 6.35$ (d, $J=3.6 \mathrm{~Hz}$, $1 \mathrm{H}), 6.22(\mathrm{~d}, J=3.6 \mathrm{~Hz}, 1 \mathrm{H}), 4.05(\mathrm{t}, J=7.3 \mathrm{~Hz}, 2 \mathrm{H}), 3.86(\mathrm{~s}, 3 \mathrm{H}), 1.18-1.13(\mathrm{~m}, 2 \mathrm{H}), 0.85-0.81$ (m, 2H), $0.56(\mathrm{t}, J=7.4 \mathrm{~Hz}, 3 \mathrm{H}) ;{ }^{13} \mathrm{C} \mathrm{NMR}\left(151 \mathrm{MHz}, \mathrm{CDCl}_{3}\right) \delta 159.0,138.7,138.6,133.9,132.3$, 130.3, 128.4, 126.0, 113.9, 111.4, 109.6, 109.6, 55.3, 45.2, 32.7, 19.3, 13.4; HRMS (ESI) m/z: [M $+\mathrm{Na}]^{+}$Calcd for $\mathrm{C}_{22} \mathrm{H}_{22} \mathrm{~N}_{2} \mathrm{ONa}$ 353.1624; Found 353.1625.

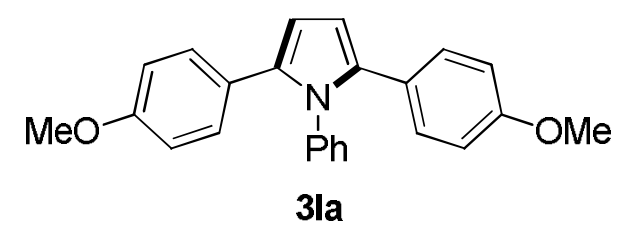

Compound 3 la $^{3}: 46 \mathrm{mg}, 52 \%$ yield, white solid; mp 203-205 ${ }^{\circ} \mathrm{C}$; Flash column chromatography conditions: petroleum ethers/EtOAc $=30: 1 ;{ }^{1} \mathrm{H}$ NMR $\left(600 \mathrm{MHz}, \mathrm{CDCl}_{3}\right) \delta 7.23-7.22(\mathrm{~m}, 3 \mathrm{H})$, 7.03-7.00 (m, 2H), 6.98-6.97 (m, 4H), 6.71 (d, $J=8.4 \mathrm{~Hz}, 4 \mathrm{H}), 6.39$ (d, $J=0.9 \mathrm{~Hz}, 2 \mathrm{H}), 3.74$ (s, $6 \mathrm{H}) ;{ }^{13} \mathrm{C}$ NMR $\left(151 \mathrm{MHz}, \mathrm{CDCl}_{3}\right) \delta 158.0,139.0,135.1,129.9,128.9,128.6,127.0,126.0,113.3$, $108.9,55.1$.

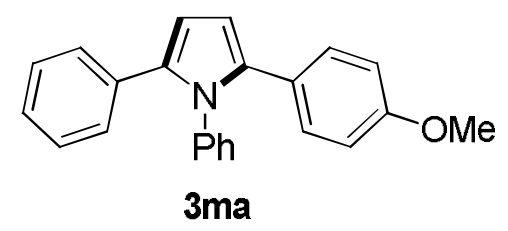

Compound 3ma: $53 \mathrm{mg}$, 65\% yield, white solid; mp 166-167 ${ }^{\circ} \mathrm{C}$; Flash column chromatography conditions: petroleum ethers/EtOAc $=50: 1 ;{ }^{1} \mathrm{H}$ NMR $\left(600 \mathrm{MHz}, \mathrm{CDCl}_{3}\right) \delta 7.23-7.21(\mathrm{~m}, 3 \mathrm{H})$, 7.15-7.14 (m, 2H), 7.13-7.10 (m, 1H), 7.07-7.04 (m, 2H), 7.02-6.95 (m, 4H), 6.75-6.70 (m, 2H), 6.48-6.45 (m, 1H), 6.43-6.39 (m, 1H), $3.74(\mathrm{~d}, J=0.8 \mathrm{~Hz}, 3 \mathrm{H}) ;{ }^{13} \mathrm{C} \mathrm{NMR}\left(151 \mathrm{MHz}, \mathrm{CDCl}_{3}\right) \delta$ 158.1, 139.0, 135.7, 135.2, 133.3, 130.0, 128.9, 128.7, 128.6, 127.8, 127.1, 126.1, 125.9, 113.3, 109.7, 109.2, 55.1; HRMS (ESI) $m / z$ : $[\mathrm{M}+\mathrm{Na}]^{+}$Calcd for $\mathrm{C}_{23} \mathrm{H}_{19} \mathrm{NONa}$ 348.1359; Found 348.1353.

\footnotetext{
${ }^{3}$ Kramer, S.; Madsen, J.; Rottlander, M.; Skrydstrup, T. Org. Lett. 2010, 12, 2758.
} 


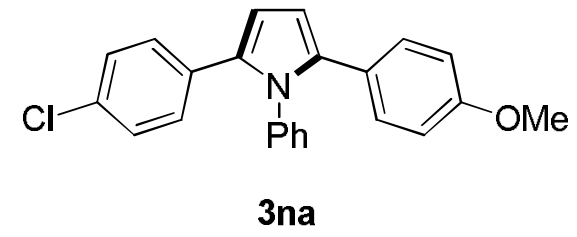

Compound 3na: $61 \mathrm{mg}, 68 \%$ yield, white solid; mp $157-159{ }^{\circ} \mathrm{C}$; Flash column chromatography conditions: petroleum ethers $/ \mathrm{EtOAc}=50: 1 ;{ }^{1} \mathrm{H}$ NMR $\left(600 \mathrm{MHz}, \mathrm{CDCl}_{3}\right) \delta 7.24(\mathrm{~d}, J=6.3 \mathrm{~Hz}, 3 \mathrm{H})$, $7.11(\mathrm{~d}, J=8.4 \mathrm{~Hz}, 2 \mathrm{H}), 7.02-6.99(\mathrm{~m}, 2 \mathrm{H}), 6.96(\mathrm{t}, J=7.9 \mathrm{~Hz}, 4 \mathrm{H}), 6.71(\mathrm{~d}, J=8.6 \mathrm{~Hz}, 2 \mathrm{H})$, $6.45(\mathrm{~d}, J=3.5 \mathrm{~Hz}, 1 \mathrm{H}), 6.39(\mathrm{~d}, J=3.5 \mathrm{~Hz}, 1 \mathrm{H}), 3.73(\mathrm{~s}, 3 \mathrm{H}) ;{ }^{13} \mathrm{C} \mathrm{NMR}\left(151 \mathrm{MHz}, \mathrm{CDCl}_{3}\right) \delta$ 158.2, 138.7, 136.1, 133.9, 131.9, 131.8, 130.0, 129.6, 128.8, 128.0, 127.3, 125.6, 113.4, 110.0, 109.3, 55.1; HRMS (ESI) $m / z$ : $[\mathrm{M}+\mathrm{Na}]^{+}$Calcd for $\mathrm{C}_{23} \mathrm{H}_{18} \mathrm{CINONa} 382.0969$; Found 382.0967.

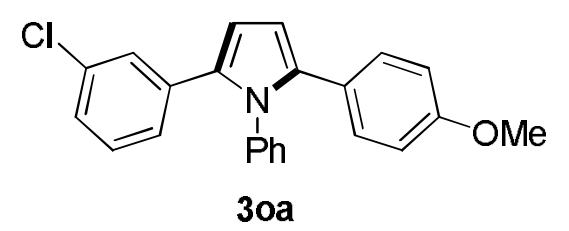

Compound 3oa: $62 \mathrm{mg}, 69 \%$ yield, white solid; mp 165-167 ${ }^{\circ} \mathrm{C}$; Flash column chromatography conditions: petroleum ethers/EtOAc $=50: 1 ;{ }^{1} \mathrm{H}$ NMR $\left(600 \mathrm{MHz}, \mathrm{CDCl}_{3}\right) \delta 7.27-7.25(\mathrm{~m}, 3 \mathrm{H})$, 7.11-7.07 (m, 2H), 7.06-7.01 (m, 3H), 6.98-6.97 (m, 2H), 6.84-6.82 (m, 1H), 6.74-6.69 (m, 2H), 6.49-6.48 (m, 1H), 6.40-6.39 (m, 1H), $3.74(\mathrm{~s}, 3 \mathrm{H}) ;{ }^{13} \mathrm{C}$ NMR $\left(151 \mathrm{MHz}, \mathrm{CDCl}_{3}\right) \delta 159.2,139.6$, 137.3, 136.0, 134.7, 134.6, 131.0, 130.0, 129.9, 129.8, 129.3, 128.4, 127.5, 127.0, 126.5, 114.7, 111.4, 110.3, 100.9, 56.1; HRMS (ESI) $\mathrm{m} / z$ : $[\mathrm{M}+\mathrm{Na}]^{+}$Calcd for $\mathrm{C}_{23} \mathrm{H}_{18} \mathrm{CINONa} 382.0969$; Found 382.0964 .

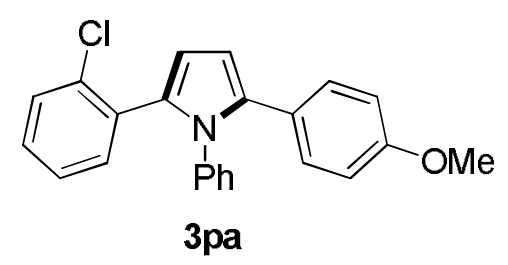

Compound 3pa: $59 \mathrm{mg}, 66 \%$ yield, white solid; mp 160-163 ${ }^{\circ} \mathrm{C}$; Flash column chromatography conditions: petroleum ethers/EtOAc $=50: 1 ;{ }^{1} \mathrm{H}$ NMR $\left(600 \mathrm{MHz}, \mathrm{CDCl}_{3}\right) \delta 7.29(\mathrm{~d}, J=7.9 \mathrm{~Hz}, 1 \mathrm{H})$, 7.15-7.10 (m, 5H), $7.06(\mathrm{t}, J=7.5 \mathrm{~Hz}, 1 \mathrm{H}), 7.04-7.00(\mathrm{~m}, 2 \mathrm{H}), 6.99-6.93(\mathrm{~m}, 2 \mathrm{H}), 6.75-6.68(\mathrm{~m}$, 2H), 6.44-6.43 (m, 2H), $3.74(\mathrm{~s}, 3 \mathrm{H}) ;{ }^{13} \mathrm{C}$ NMR (151 MHz, $\left.\mathrm{CDCl}_{3}\right) \delta 158.0,138.7,134.8,134.7$, 
$133.2,132.7,131.8,129.7,129.3,128.6,128.4,128.3,126.8,125.9,125.8,113.4,110.9,108.7$, 55.1; HRMS (ESI) $m / z$ : [M + H] $]^{+}$Calcd for $\mathrm{C}_{23} \mathrm{H}_{19} \mathrm{ClNO} 360.1150$; Found 360.1159 .

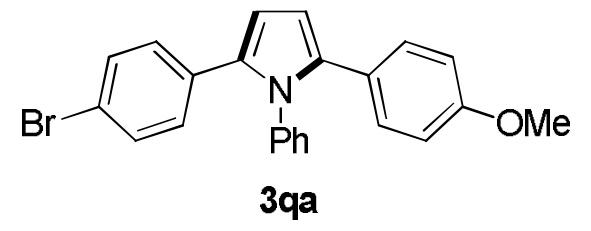

Compound 3qa: $75 \mathrm{mg}$, $75 \%$ yield, white solid; mp $170-173{ }^{\circ} \mathrm{C}$; Flash column chromatography conditions: petroleum ethers/EtOAc $=50: 1 ;{ }^{1} \mathrm{H} \mathrm{NMR}\left(600 \mathrm{MHz}, \mathrm{CDCl}_{3}\right) \delta 7.26(\mathrm{t}, J=9.0 \mathrm{~Hz}, 5 \mathrm{H})$, $7.01(\mathrm{~d}, J=6.0 \mathrm{~Hz}, 2 \mathrm{H}), 6.97(\mathrm{~d}, J=8.4 \mathrm{~Hz}, 2 \mathrm{H}), 6.90(\mathrm{~d}, J=8.2 \mathrm{~Hz}, 2 \mathrm{H}), 6.72(\mathrm{~d}, J=8.4 \mathrm{~Hz}$, 2H), $6.46(\mathrm{~d}, J=3.3 \mathrm{~Hz}, 1 \mathrm{H}), 6.40(\mathrm{~d}, J=3.3 \mathrm{~Hz}, 1 \mathrm{H}), 3.75(\mathrm{~s}, 3 \mathrm{H}) ;{ }^{13} \mathrm{C} \mathrm{NMR}\left(151 \mathrm{MHz}, \mathrm{CDCl}_{3}\right)$ $\delta 158.2,138.7,136.2,133.9,132.2,131.0,129.9,130.0,128.9,128.8,127.4,125.6,120.1,113.4$, 110.0, 109.3, 55.1; HRMS (ESI) $m / z:[\mathrm{M}+\mathrm{Na}]^{+}$Calcd for $\mathrm{C}_{23} \mathrm{H}_{18} \mathrm{BrNONa}$ 426.0464; Found 426.0457.

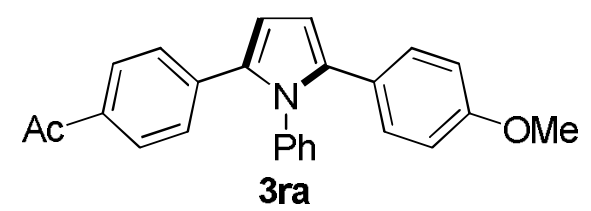

Compound 3ra: $64 \mathrm{mg}, 70 \%$ yield, white solid; mp 213-216 ${ }^{\circ} \mathrm{C}$; Flash column chromatography conditions: petroleum ethers/EtOAc $=20: 1 ;{ }^{1} \mathrm{H}$ NMR $\left(600 \mathrm{MHz}, \mathrm{CDCl}_{3}\right) \delta 7.77(\mathrm{~d}, J=8.3 \mathrm{~Hz}$, 2H), 7.32-7.30 (m, 3H), 7.14 (d, $J=8.3 \mathrm{~Hz}, 2 \mathrm{H}), 7.08$ (d, $J=6.9 \mathrm{~Hz}, 2 \mathrm{H}), 7.01$ (d, $J=8.6 \mathrm{~Hz}$, 2H), $6.76(\mathrm{~d}, J=8.6 \mathrm{~Hz}, 2 \mathrm{H}), 6.63(\mathrm{~d}, J=3.5 \mathrm{~Hz}, 1 \mathrm{H}), 6.46$ (d, $J=3.5 \mathrm{~Hz}, 1 \mathrm{H}), 3.79$ (s, 3H), 2.56 (s, 3H); ${ }^{13} \mathrm{C}$ NMR $\left(151 \mathrm{MHz}, \mathrm{CDCl}_{3}\right) \delta 197.6,158.4,138.8,137.9,137.2,134.2,133.9,130.1$, 121.0, 128.8, 128.1, 127.9, 127.6, 125.4, 113.4, 111.4, 109.7, 55.4, 26.5; HRMS (ESI) $m / z:[\mathrm{M}+$ $\mathrm{Na}]^{+}$Calcd for $\mathrm{C}_{25} \mathrm{H}_{21} \mathrm{NO}_{2} \mathrm{Na}$ 390.1465; Found 390.1461.

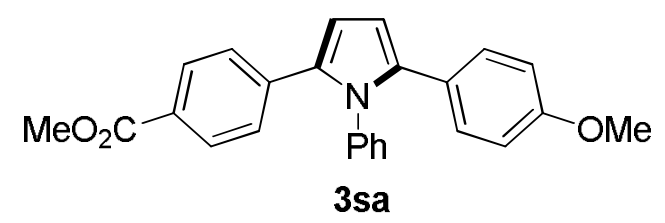

Compound 3sa: $76 \mathrm{mg}, 80 \%$ yield, white solid; mp $233-236{ }^{\circ} \mathrm{C}$; Flash column chromatography conditions: petroleum ethers/EtOAc $=10: 1 ;{ }^{1} \mathrm{H}$ NMR $\left(600 \mathrm{MHz}, \mathrm{CDCl}_{3}\right) \delta 7.81(\mathrm{~d}, J=8.3 \mathrm{~Hz}, 2 \mathrm{H})$, 
7.28-7.22 (m, 3H), 7.09 (d, $J=8.3 \mathrm{~Hz}, 2 \mathrm{H}), 7.03(\mathrm{~d}, J=7.9 \mathrm{~Hz}, 2 \mathrm{H}), 6.98(\mathrm{~d}, J=8.6 \mathrm{~Hz}, 2 \mathrm{H})$, $6.72(\mathrm{~d}, J=8.6 \mathrm{~Hz}, 2 \mathrm{H}), 6.58(\mathrm{~d}, J=3.6 \mathrm{~Hz}, 1 \mathrm{H}), 6.42(\mathrm{~d}, J=3.6 \mathrm{~Hz}, 1 \mathrm{H}), 3.86(\mathrm{~s}, 3 \mathrm{H}), 3.75(\mathrm{~s}$, $3 \mathrm{H}) ;{ }^{13} \mathrm{C} \mathrm{NMR}\left(151 \mathrm{MHz}, \mathrm{CDCl}_{3}\right) \delta 167.0,158.4,138.7,137.7,137.0,134.0,130.1,129.2,128.9$, $128.8,127.8,127.5,127.2,125.4,113.4,111.1,109.6,55.1,51.9$; HRMS (ESI) $m / z:[\mathrm{M}+\mathrm{Na}]^{+}$ Calcd for $\mathrm{C}_{25} \mathrm{H}_{21} \mathrm{NO}_{3} \mathrm{Na}$ 406.1414; Found 406.1418.

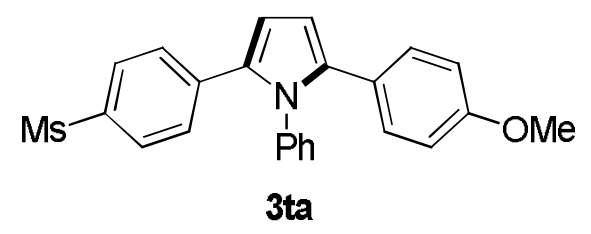

Compound 3ta: $81 \mathrm{mg}, 81 \%$ yield, white solid; $\mathrm{mp} 246-249{ }^{\circ} \mathrm{C}$; Flash column chromatography conditions: petroleum ethers/EtOAc $=5: 1 ;{ }^{1} \mathrm{H}$ NMR $\left(600 \mathrm{MHz}, \mathrm{CDCl}_{3}\right) \delta 7.72-7.68(\mathrm{~m}, 2 \mathrm{H})$, 7.34-7.27 (m, 3H), 7.21-7.17 (m, 2H), 7.06-7.02 (m, 2H), 7.00-6.96 (m, 2H), 6.75-6.72 (m, 2H), $6.61(\mathrm{~d}, J=3.7 \mathrm{~Hz}, 1 \mathrm{H}), 6.44(\mathrm{~d}, J=3.7 \mathrm{~Hz}, 1 \mathrm{H}), 3.76(\mathrm{~s}, 3 \mathrm{H}), 3.02(\mathrm{~s}, 3 \mathrm{H}) ;{ }^{13} \mathrm{C} \mathrm{NMR}(151 \mathrm{MHz}$, $\left.\mathrm{CDCl}_{3}\right) \delta 158.5,138.6,138.5,137.7,137.1,132.9,130.1,129.2,128.8,128.3,127.8,127.0,125.2$, 113.5, 111.9, 109.9, 55.2, 44.3; HRMS (ESI) $m / z$ : $[\mathrm{M}+\mathrm{Na}]^{+}$Calcd for $\mathrm{C}_{24} \mathrm{H}_{21} \mathrm{NSO}_{3} \mathrm{Na}$ 426.1134; Found 426.1134.

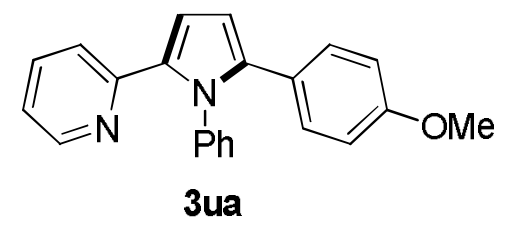

Compound 3ua: $68 \mathrm{mg}, 84 \%$ yield, white solid; mp 203-205 ${ }^{\circ} \mathrm{C}$; Flash column chromatography conditions: petroleum ethers/EtOAc $=10: 1 ;{ }^{1} \mathrm{H}$ NMR $\left(600 \mathrm{MHz}, \mathrm{CDCl}_{3}\right) \delta 8.45(\mathrm{~d}, J=4.3 \mathrm{~Hz}$, 1H), 7.39-7.36 (m, 1H), 7.28-7.25 (m, 3H), 7.20-7.05 (m, 2H), 7.01-6.95 (m, 3H), $6.84(\mathrm{~d}, J=$ $3.7 \mathrm{~Hz}, 1 \mathrm{H}), 6.80(\mathrm{~d}, J=8.0 \mathrm{~Hz}, 1 \mathrm{H}), 6.72(\mathrm{~d}, J=8.8 \mathrm{~Hz}, 2 \mathrm{H}), 6.42(\mathrm{~d}, J=3.7 \mathrm{~Hz}, 1 \mathrm{H}), 3.75(\mathrm{~s}$, $3 \mathrm{H}),{ }^{13} \mathrm{C}$ NMR $\left(151 \mathrm{MHz}, \mathrm{CDCl}_{3}\right) \delta 158.3,151.7,149.1,139.4,137.3,135.4,134.5,130.1,128.8$, 128.7, 127.3, 125.7, 122.2, 120.4, 113.3, 112.1, 109.7, 55.1; HRMS (ESI) $m / z:[\mathrm{M}+\mathrm{Na}]^{+} \mathrm{Calcd}$ for $\mathrm{C}_{22} \mathrm{H}_{18} \mathrm{~N}_{2} \mathrm{ONa}$ 349.1311; Found 349.1318. 


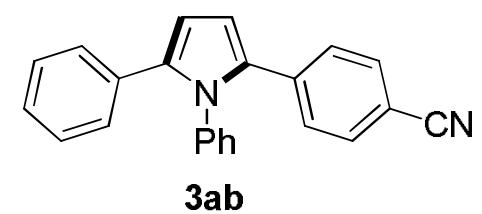

Compound 3ab: $56 \mathrm{mg}, 70 \%$ yield, white solid; mp $141-145{ }^{\circ} \mathrm{C}$; Flash column chromatography conditions: petroleum ethers/EtOAc $=50: 1 ;{ }^{1} \mathrm{H}$ NMR $\left(600 \mathrm{MHz}, \mathrm{CDCl}_{3}\right) \delta 7.44-7.41(\mathrm{~m}, 2 \mathrm{H})$, 7.33-7.27 (m, 3H), 7.20-7.16 (m, 3H), 7.12-7.09 (m, 2H), 7.07-7.02 (m, 4H), 6.61 (d, J=3.8 Hz, 1H), $6.50(\mathrm{~d}, J=3.7 \mathrm{~Hz}, 1 \mathrm{H}) ;{ }^{13} \mathrm{C} \mathrm{NMR}\left(151 \mathrm{MHz}, \mathrm{CDCl}_{3}\right) \delta 138.4,137.7,137.6,133.5,132.6$, $131.8,129.2,128.9,128.8,128.3,128.0,127.9,126.8,119.2,112.0,110.6,109.1$; HRMS (ESI) $m / z:[\mathrm{M}+\mathrm{H}]^{+}$Calcd for $\mathrm{C}_{23} \mathrm{H}_{17} \mathrm{~N}_{2} 321.1386$; Found 321.1380 .

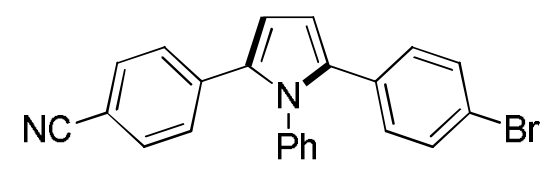

3ac

Compound 3ac: $50 \mathrm{mg}$, 51\% yield, white solid; mp 168-170 ${ }^{\circ} \mathrm{C}$; Flash column chromatography conditions: petroleum ethers/EtOAc $=50: 1 ;{ }^{1} \mathrm{H}$ NMR $\left(600 \mathrm{MHz}, \mathrm{CDCl}_{3}\right) \delta 7.43(\mathrm{~d}, J=8.5 \mathrm{~Hz}, 2 \mathrm{H})$, 7.37-7.28 (m, 5H), 7.10 (d, $J=8.5 \mathrm{~Hz}, 2 \mathrm{H}), 7.05-6.99(\mathrm{~m}, 2 \mathrm{H}), 6.90$ (d, $J=8.5 \mathrm{~Hz}, 2 \mathrm{H}), 6.59$ (d, $J=3.8 \mathrm{~Hz}, 1 \mathrm{H}), 6.50(\mathrm{~d}, J=3.8 \mathrm{~Hz}, 1 \mathrm{H}) ;{ }^{13} \mathrm{C} \mathrm{NMR}\left(151 \mathrm{MHz}, \mathrm{CDCl}_{3}\right) \delta 138.1,137.4,136.4$, $136.3,133.9,131.8,131.5,131.2,130.2,129.3,128.6,128.3,128.1,120.9,112.0,110.8,109.3$; HRMS (ESI) $m / z$ : $[\mathrm{M}+\mathrm{Na}]^{+}$Calcd for $\mathrm{C}_{23} \mathrm{H}_{15} \mathrm{BrN}_{2} \mathrm{Na}$ 421.0311; Found 421.0315.

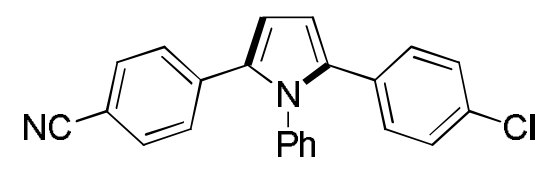

3ad

Compound 3ad: $47 \mathrm{mg}$, 54\% yield, yellow solid; mp 153-155 ${ }^{\circ} \mathrm{C}$; Flash column chromatography conditions: petroleum ethers/EtOAc $=50: 1 ;{ }^{1} \mathrm{H}$ NMR $\left(600 \mathrm{MHz}, \mathrm{CDCl}_{3}\right) \delta 7.47-7.44(\mathrm{~m}, 2 \mathrm{H})$, 7.39-7.31 (m, 3H), 7.20-7.16 (m, 2H), 7.15-7.11 (m, 2H), 7.07-7.03 (m, 2H), 7.01-6.97 (m, 2H), $6.62(\mathrm{~d}, J=3.8 \mathrm{~Hz}, 1 \mathrm{H}), 6.52(\mathrm{~d}, J=3.8 \mathrm{~Hz}, 1 \mathrm{H}) ;{ }^{13} \mathrm{C} \mathrm{NMR}\left(151 \mathrm{MHz}, \mathrm{CDCl}_{3}\right) \delta 138.1,137.3$, $136.3,133.8,132.7,131.7,131.0,129.8,129.3,128.6,128.3,128.2,128.0,119.0,111.9,110.7$, 109.3; HRMS (ESI) $m / z$ : $[\mathrm{M}+\mathrm{Na}]^{+}$Calcd for $\mathrm{C}_{23} \mathrm{H}_{15} \mathrm{ClN}_{2} \mathrm{Na}$ 377.0816; Found 377.0812 . 


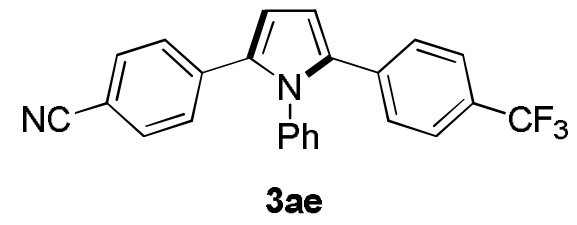

Compound 3ae: $63 \mathrm{mg}$, 65\% yield, yellow solid; mp 223-225 ${ }^{\circ} \mathrm{C}$; Flash column chromatography conditions: petroleum ethers/EtOAc $=50: 1 ;{ }^{1} \mathrm{H}$ NMR $\left(600 \mathrm{MHz}, \mathrm{CDCl}_{3}\right) \delta 7.48-7.41(\mathrm{~m}, 4 \mathrm{H})$, $7.36(\mathrm{~d}, J=7.2 \mathrm{~Hz}, 1 \mathrm{H}), 7.33$ (t, $J=7.4 \mathrm{~Hz}, 2 \mathrm{H}), 7.17-7.10(\mathrm{~m}, 4 \mathrm{H}), 7.05(\mathrm{~d}, J=7.5 \mathrm{~Hz}, 2 \mathrm{H})$, $6.61(\mathrm{~d}, J=3.8 \mathrm{~Hz}, 1 \mathrm{H}), 6.58(\mathrm{~d}, J=3.8 \mathrm{~Hz}, 1 \mathrm{H}) ;{ }^{13} \mathrm{C} \mathrm{NMR}\left(151 \mathrm{MHz}, \mathrm{CDCl}_{3}\right) \delta 138.0,137.2$, $136.0,135.8,134.5,131.8,129.4,128.7,128.6,128.5(\mathrm{q}, J=32.2 \mathrm{~Hz}), 128.4,128.2,125.0(\mathrm{q}, J=$ $3.7 \mathrm{~Hz}), 124.1(\mathrm{q}, J=272.0 \mathrm{~Hz}), 118.9,112.0,111.6,109.5 ;{ }^{19} \mathrm{~F}$ NMR $\left(565 \mathrm{MHz}, \mathrm{CDCl}_{3}\right) \delta-62.5$; HRMS (ESI) $m / z$ : $[\mathrm{M}+\mathrm{Na}]^{+}$Calcd for $\mathrm{C}_{24} \mathrm{H}_{15} \mathrm{~N}_{2} \mathrm{~F}_{3} \mathrm{Na}$ 411.1080; Found 411.1069.

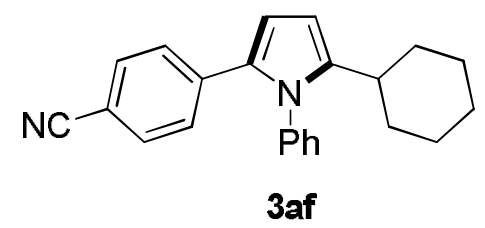

Compound 3af: $51 \mathrm{mg}, 63 \%$ yield, white solid; $\mathrm{mp} 141-143{ }^{\circ} \mathrm{C}$; Flash column chromatography conditions: petroleum ethers/EtOAc $=50: 1 ;{ }^{1} \mathrm{H}$ NMR $\left(600 \mathrm{MHz}, \mathrm{CDCl}_{3}\right) \delta 7.44-7.39(\mathrm{~m}, 3 \mathrm{H})$, $7.35(\mathrm{~d}, J=8.4 \mathrm{~Hz}, 2 \mathrm{H}), 7.19-7.15(\mathrm{~m}, 2 \mathrm{H}), 7.05(\mathrm{~d}, J=8.4 \mathrm{~Hz}, 2 \mathrm{H}), 6.52(\mathrm{~d}, J=3.7 \mathrm{~Hz}, 1 \mathrm{H})$, $6.15(\mathrm{~d}, J=3.7 \mathrm{~Hz}, 1 \mathrm{H}), 2.40-2.35(\mathrm{~m}, 1 \mathrm{H}), 1.84-1.76(\mathrm{~m}, 2 \mathrm{H}), 1.74-1.68(\mathrm{~m}, 2 \mathrm{H}), 1.68-1.63(\mathrm{~m}$, 1H), 1.38-1.31 (m, 2H), 1.21-1.09 (m, 3H) ${ }^{13} \mathrm{C}$ NMR (151 MHz, $\left.\mathrm{CDCl}_{3}\right) \delta$ 144.7, 138.7, 137.7, 131.7, 131.6, 129.3, 128.6, 128.1, 127.3, 119.2, 111.2, 108.2, 105.3, 35.7, 33.9, 26.4, 25.9; HRMS (ESI) $m / z:[\mathrm{M}+\mathrm{Na}]^{+}$Calcd for $\mathrm{C}_{23} \mathrm{H}_{22} \mathrm{~N}_{2} \mathrm{Na}$ 349.1675; Found 349.1681.

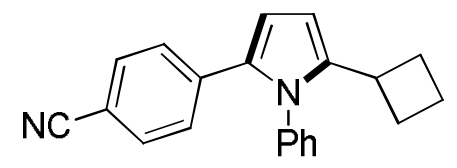

3ag

Compound 3ag: $30 \mathrm{mg}, 40 \%$ yield, white solid; $\mathrm{mp} 130-132{ }^{\circ} \mathrm{C}$; Flash column chromatography conditions: petroleum ethers/EtOAc $=50: 1 ;{ }^{1} \mathrm{H}$ NMR $\left(600 \mathrm{MHz}, \mathrm{CDCl}_{3}\right) \delta 7.44-7.35(\mathrm{~m}, 5 \mathrm{H})$, 7.14-7.12 (m, 2H), $7.07(\mathrm{~d}, J=8.4 \mathrm{~Hz}, 2 \mathrm{H}), 6.54(\mathrm{~d}, J=3.6 \mathrm{~Hz}, 1 \mathrm{H}), 6.24(\mathrm{~d}, J=3.6 \mathrm{~Hz}, 1 \mathrm{H})$, 3.31-3.21 (m, 1H), 2.14-2.01 (m, 4H), 1.85-1.77 (m, 2H) ${ }^{13} \mathrm{C}^{\mathrm{NMR}}\left(151 \mathrm{MHz}, \mathrm{CDCl}_{3}\right) \delta 143.3$, 
138.9, 137.6, 132.1, 131.8, 129.2, 128.3, 128.0, 127.2, 119.2, 111.0, 108.3, 105.9, 32.8, 29.5, 18.3; HRMS (ESI) $m / z$ : [M + H] Calcd for $\mathrm{C}_{21} \mathrm{H}_{19} \mathrm{~N}_{2}$ 299.1543; Found 299.1534.

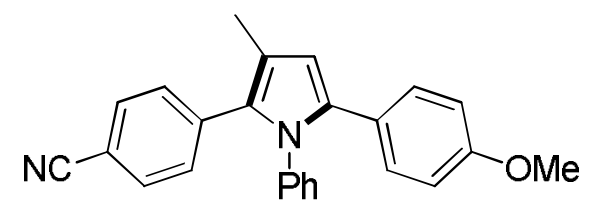

\section{3ah}

Compound 3ah: $61 \mathrm{mg}, 67 \%$ yield, white solid; mp $213-215{ }^{\circ} \mathrm{C}$; Flash column chromatography conditions: petroleum ethers/EtOAc $=50: 1 ;{ }^{1} \mathrm{H}$ NMR $\left(600 \mathrm{MHz}, \mathrm{CDCl}_{3}\right) \delta 7.45(\mathrm{~d}, J=8.4 \mathrm{~Hz}, 2 \mathrm{H})$, $7.23-7.19(\mathrm{~m}, 3 \mathrm{H}), 7.09(\mathrm{~d}, J=8.4 \mathrm{~Hz}, 2 \mathrm{H}), 6.96(\mathrm{~d}, J=8.8 \mathrm{~Hz}, 2 \mathrm{H}), 6.93-6.91(\mathrm{~m}, 2 \mathrm{H})$, 6.73-6.71 (m, 2H), $6.32(\mathrm{~s}, 1 \mathrm{H}), 3.75(\mathrm{~s}, 3 \mathrm{H}), 2.22(\mathrm{~s}, 3 \mathrm{H}){ }^{13} \mathrm{C} \mathrm{NMR}\left(151 \mathrm{MHz}, \mathrm{CDCl}_{3}\right) \delta 158.4$, 138.7, 137.5, 135.5, 131.4, 130.4, 129.9, 128.8, 128.7, 127.2, 125.2, 119.8, 119.1,113.4,111.7, 109.1, 99.9, 55.1, 12.2; HRMS (ESI) $m / z$ : $[\mathrm{M}+\mathrm{Na}]^{+}$Calcd for $\mathrm{C}_{25} \mathrm{H}_{20} \mathrm{~N}_{2} \mathrm{ONa}$ 387.1468; Found 387.1460.

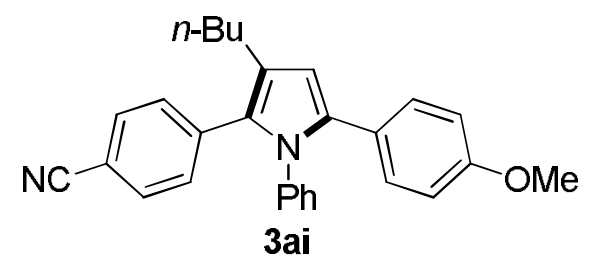

Compound 3ai: $63 \mathrm{mg}, 62 \%$ yield, white solid; mp $223-225{ }^{\circ} \mathrm{C}$; Flash column chromatography conditions: petroleum ethers/EtOAc $=50: 1 ;{ }^{1} \mathrm{H}$ NMR $\left(600 \mathrm{MHz}, \mathrm{CDCl}_{3}\right) \delta 7.49(\mathrm{~d}, J=8.1 \mathrm{~Hz}$, 2H), 7.25-7.20 (m, 3H), 7.16-7.12 (m, 2H), 7.04-7.00 (m, 2H), 6.98-6.94 (m, 2H), $6.76(\mathrm{~d}, J=$ $8.5 \mathrm{~Hz}, 2 \mathrm{H}), 6.40(\mathrm{~d}, J=1.4 \mathrm{~Hz}, 1 \mathrm{H}), 3.78(\mathrm{~s}, 3 \mathrm{H}), 2.57$ (t, $J=7.3 \mathrm{~Hz}, 2 \mathrm{H}), 1.67$ (t, $J=6.9 \mathrm{~Hz}$, 2H), 1.46-1.40 (m, 2H), 0.97-0.93 (m, 3H); ${ }^{13} \mathrm{C} \mathrm{NMR}\left(151 \mathrm{MHz}, \mathrm{CDCl}_{3}\right) \delta 158.2,138.6,137.7$, 135.4, 131.3, 130.7, 129.8, 129.5, 128.7, 128.7, 127.0, 125.3, 125.2, 119.0, 113.4, 110.1, 109.2, 55.1, 33.4, 26.1, 22.7, 13.9; HRMS (ESI) $m / z$ : $[\mathrm{M}+\mathrm{Na}]^{+}$Calcd for $\mathrm{C}_{28} \mathrm{H}_{26} \mathrm{~N}_{2} \mathrm{ONa}$ 429.1937; Found 429.1931. 
The Three-Component Reaction

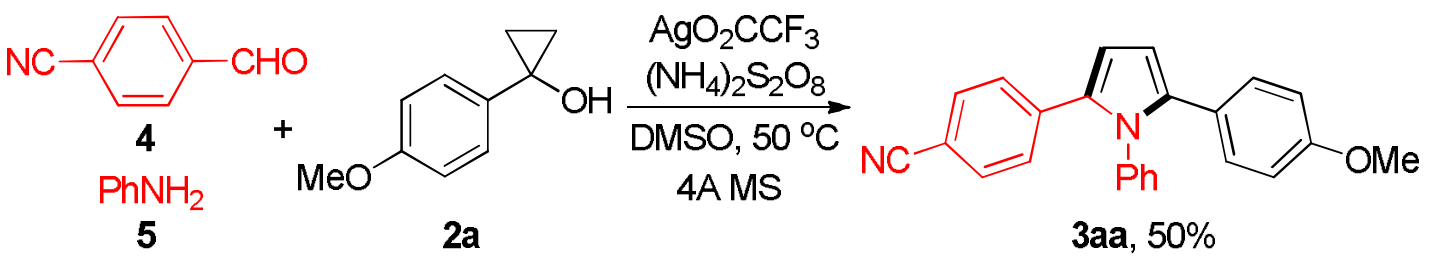

To a solution of 4 (32.8 $\mathrm{mg}, 0.25 \mathrm{mmol})$ and $\mathrm{PhNH}_{2}(23.3 \mathrm{mg}, 0.25 \mathrm{mmol})$ in $2 \mathrm{~mL}$ of dry DMSO was added $100 \mathrm{mg}$ of $4 \mathrm{~A}$ MS. After stirring at $50{ }^{\circ} \mathrm{C}$ for $0.5 \mathrm{~h}$, a solution of $2 \mathrm{a}(82.0 \mathrm{mg}, 0.5$ mmol) in $4 \mathrm{~mL}$ of dry DMSO was added, followed by the addition of $\mathrm{AgO}_{2} \mathrm{CCF}_{3}(5.5 \mathrm{mg}, 0.025$ mmol) and $\left(\mathrm{NH}_{4}\right)_{2} \mathrm{~S}_{2} \mathrm{O}_{8}(171.0 \mathrm{mg}, 0.75 \mathrm{mmol})$ under a nitrogen atmosphere. After stirring at $50{ }^{\circ} \mathrm{C}$ for 10 hours, the reaction mixture was quenched by water, extracted by EtOAc, dried by anhydrous $\mathrm{Na}_{2} \mathrm{SO}_{4}$, and concentrated. Column chromatography on silica gel (petroleum ethers/EtOAc $=50: 1)$ gave $44 \mathrm{mg}(50 \%$ yield $)$ of $\mathbf{3 a a}$ as a yellow solid.

\section{Effects of 1,1-Diphenylethylene on the Ag-Catalyzed ORA to Imines}

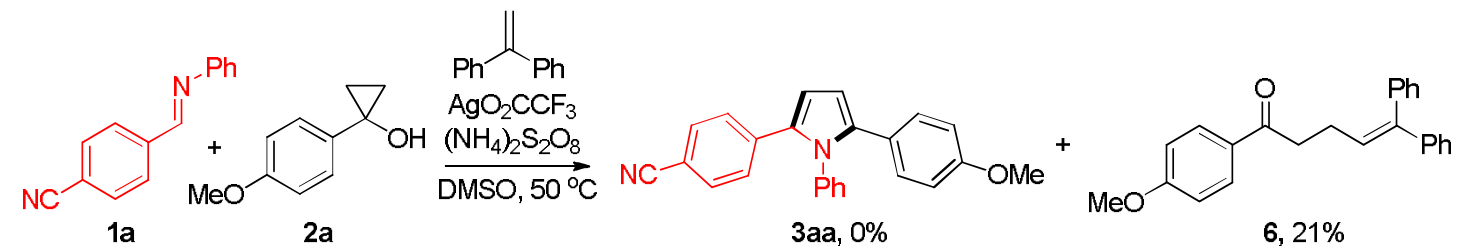

To a solution of 1a $(51.5 \mathrm{mg}, 0.25 \mathrm{mmol}), \mathbf{2 a}(82.0 \mathrm{mg}, 0.5 \mathrm{mmol})$, and 1,1-diphenylethylene (90.0 mg, $0.5 \mathrm{mmol})$ in $6 \mathrm{~mL}$ of dry DMSO was added $\mathrm{AgO}_{2} \mathrm{CCF}_{3}(5.5 \mathrm{mg}, 0.025 \mathrm{mmol})$ and $\left(\mathrm{NH}_{4}\right)_{2} \mathrm{~S}_{2} \mathrm{O}_{8}(171.0 \mathrm{mg}, 0.75 \mathrm{mmol})$ under a nitrogen atmosphere. After stirring at $50{ }^{\circ} \mathrm{C}$ for 10 hours, the reaction mixture was quenched by water, extracted by EtOAc, dried by anhydrous $\mathrm{Na}_{2} \mathrm{SO}_{4}$, and concentrated. Column chromatography on silica gel (petroleum ethers/EtOAc $=50: 1$ ) gave $18 \mathrm{mg}$ (21\% yield) of 6 as a yellow solid; mp $174-176{ }^{\circ} \mathrm{C} ;{ }^{1} \mathrm{H}$ NMR $\left(400 \mathrm{MHz}, \mathrm{CDCl}_{3}\right) \delta$ $7.92(\mathrm{~d}, J=8.8 \mathrm{~Hz}, 2 \mathrm{H}), 7.43-7.31(\mathrm{~m}, 4 \mathrm{H}), 7.27-7.20(\mathrm{~m}, 6 \mathrm{H}), 6.93(\mathrm{~d}, J=8.9 \mathrm{~Hz}, 2 \mathrm{H}), 6.18$ (t, $J$ $=7.5 \mathrm{~Hz}, 1 \mathrm{H}), 3.89(\mathrm{~s}, 3 \mathrm{H}), 3.05(\mathrm{t}, J=7.4 \mathrm{~Hz}, 2 \mathrm{H}), 2.59-2.54(\mathrm{~m}, 2 \mathrm{H}) ;{ }^{13} \mathrm{C}$ NMR $(151 \mathrm{MHz}$, $\left.\mathrm{CDCl}_{3}\right) \delta 198.0,163.4,142.6,142.4,139.9,130.3,129.8,128.3,128.1,127.2,113.7,55.4,38.4$, 24.9; HRMS (ESI) $m / z$ : $[\mathrm{M}+\mathrm{Na}]^{+}$Calcd for $\mathrm{C}_{24} \mathrm{H}_{22} \mathrm{NaO}_{2}$ 365.1512; Found 365.1507. 


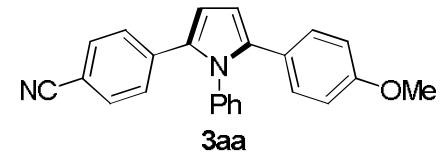

${ }^{1} \mathrm{H}$ NMR (600 MHz, $\mathrm{CDCl}_{3}$ )

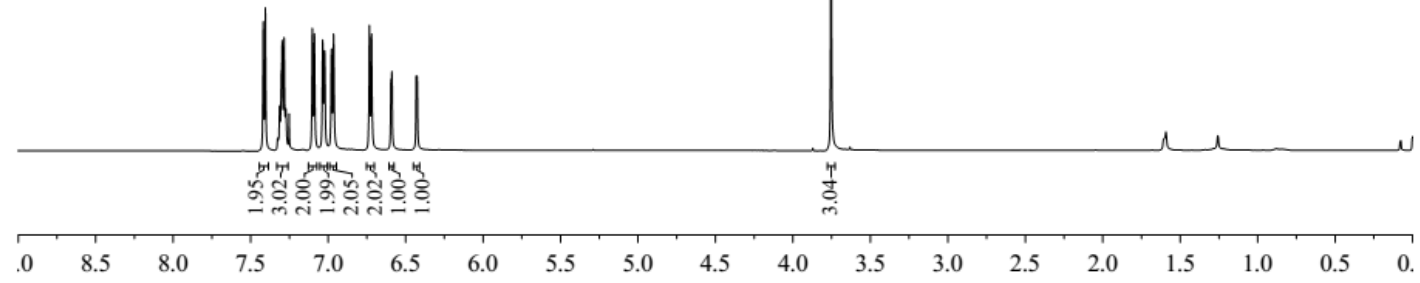

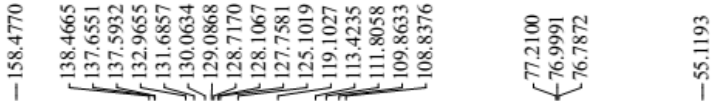<smiles>COc1ccc(-c2ccc(-c3ccc(C#N)cc3)n2-c2ccccc2)cc1</smiles>

$\left.{ }^{13} \mathrm{C} \mathrm{NMR} \mathrm{(151} \mathrm{MHz,} \mathrm{CDCl}_{3}\right)$

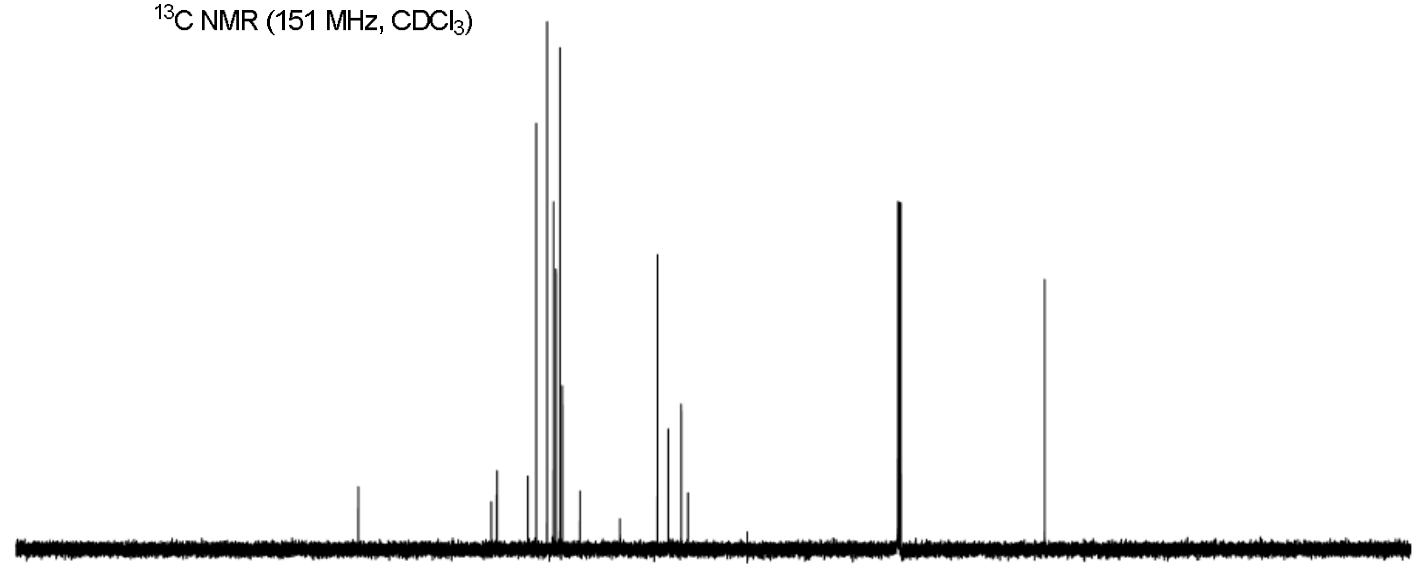

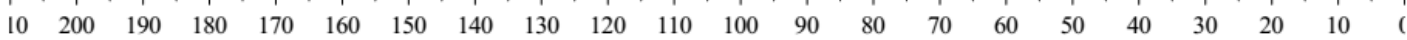




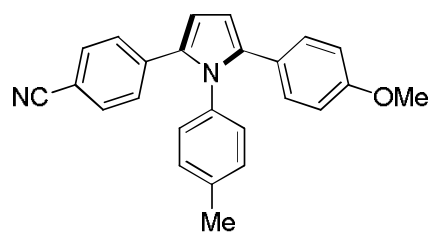

3 ba

${ }^{1} \mathrm{H}$ NMR $\left(600 \mathrm{MHz}, \mathrm{CDCl}_{3}\right)$

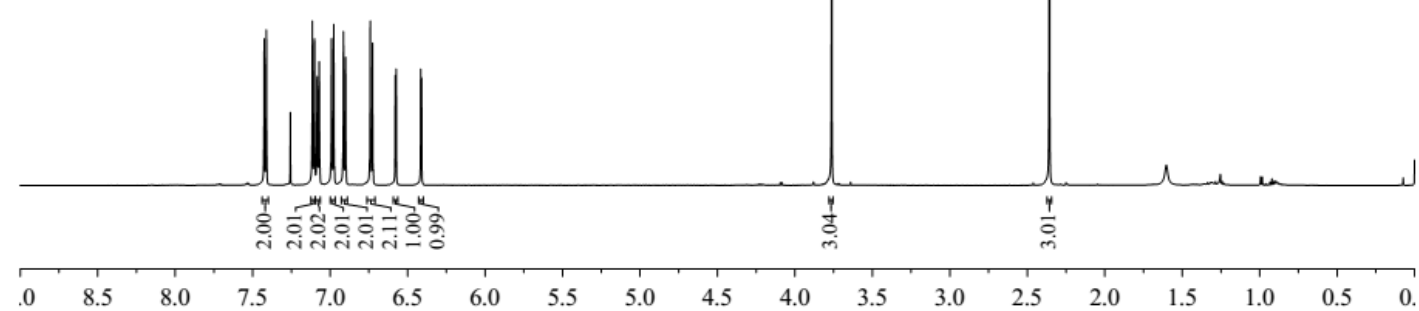

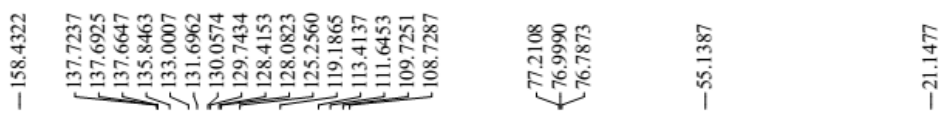<smiles>COc1ccc(-c2ccc(-c3ccc(C)cc3)n2-c2ccc(C)cc2)cc1</smiles>

${ }^{13} \mathrm{C}$ NMR (151 MHz, $\mathrm{CDCl}_{3}$ )

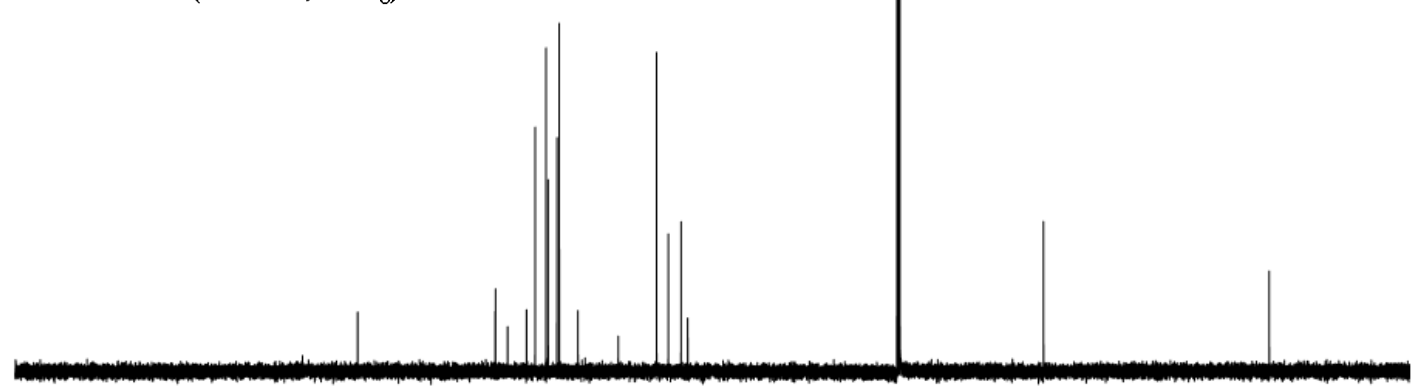

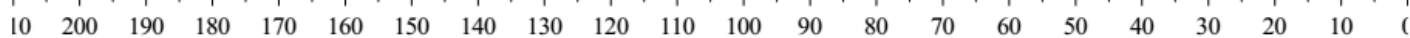



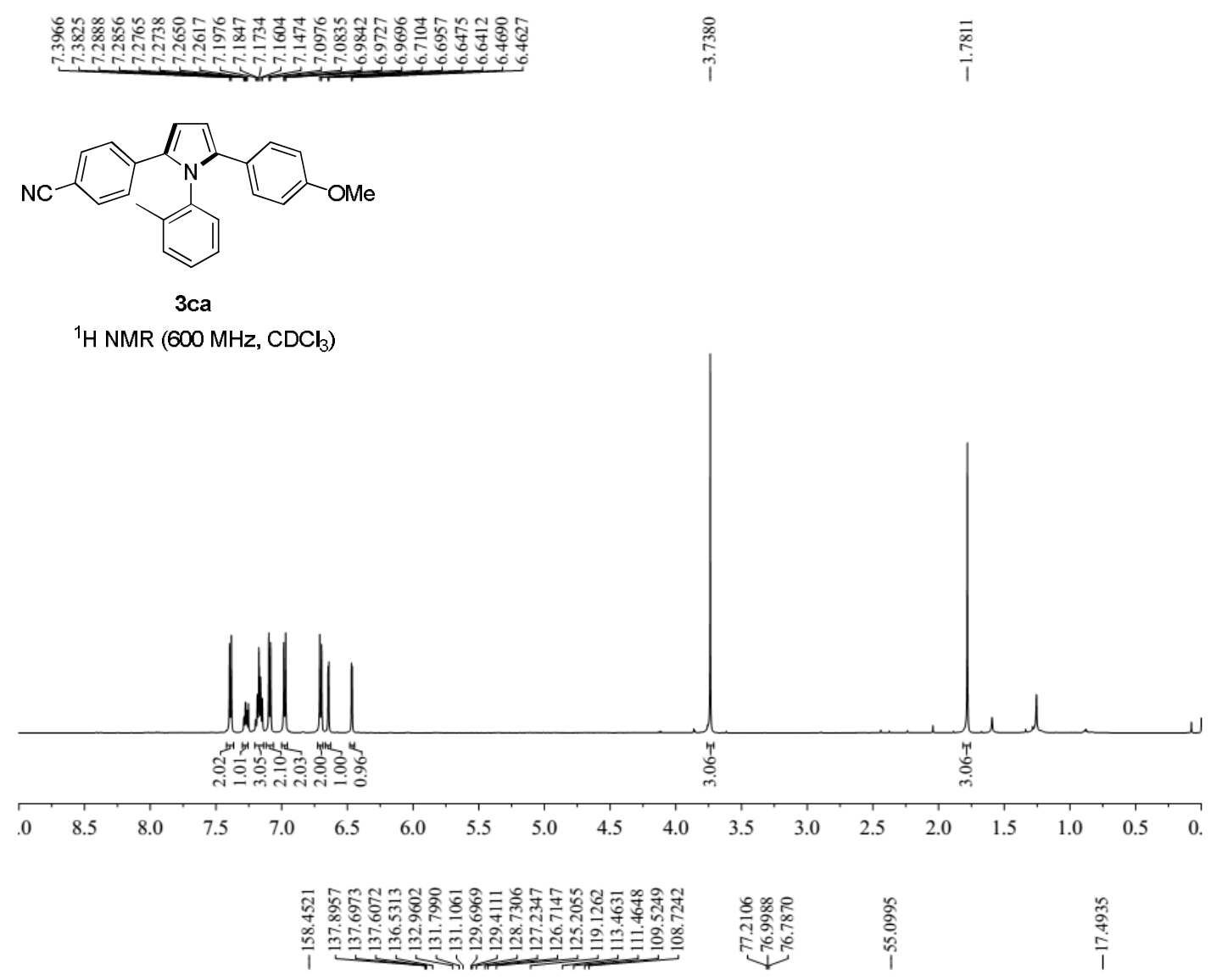<smiles>COc1ccc(-c2ccc3c4ccc(C#N)cc4c4ccccc4n23)cc1</smiles>

3ca

${ }^{13} \mathrm{C}$ NMR $\left(151 \mathrm{MHz}, \mathrm{CDCl}_{3}\right)$

$\begin{array}{lllllllllllllllllllll}10 & 200 & 190 & 180 & 170 & 160 & 150 & 140 & 130 & 120 & 110 & 100 & 90 & 80 & 70 & 60 & 50 & 40 & 30 & 20 & 10\end{array}$ 


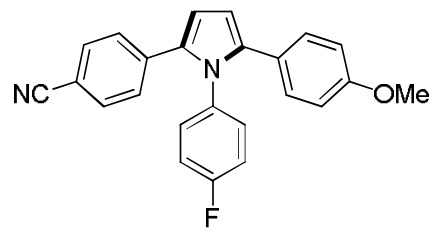

3da

${ }^{1} \mathrm{H} \mathrm{NMR}\left(400 \mathrm{MHz}, \mathrm{CDCl}_{3}\right)$

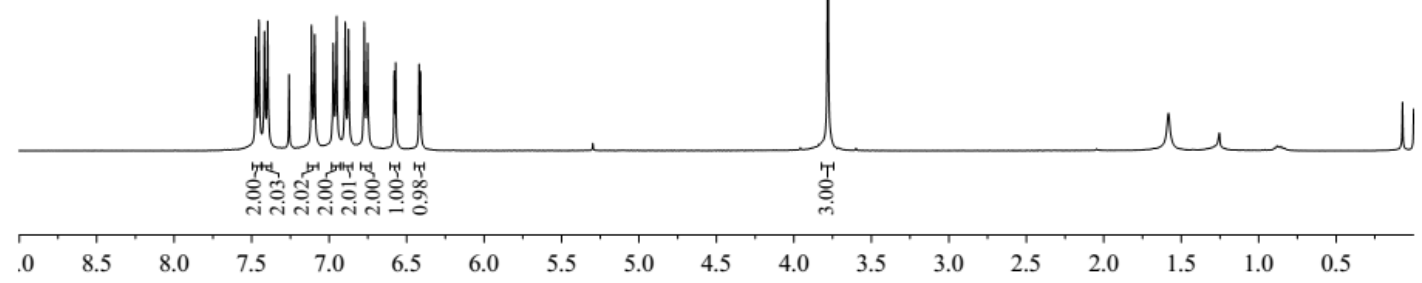

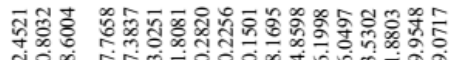

สํํ의

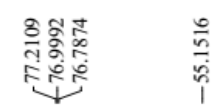

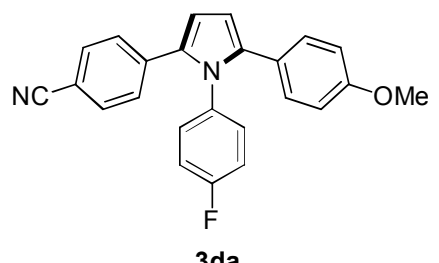

${ }^{13} \mathrm{C}$ NMR (151 MHz, $\left.\mathrm{CDCl}_{3}\right)$

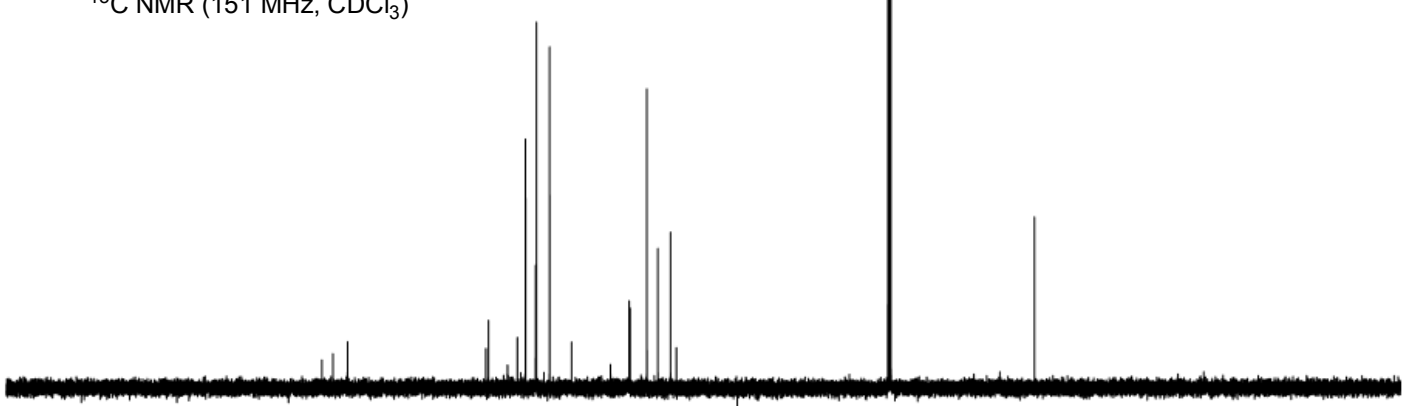

$\begin{array}{lllllllllllllllllllll}10 & 200 & 190 & 180 & 170 & 160 & 150 & 140 & 130 & 120 & 110 & 100 & 90 & 80 & 70 & 60 & 50 & 40 & 30 & 20 & 10\end{array}$ 

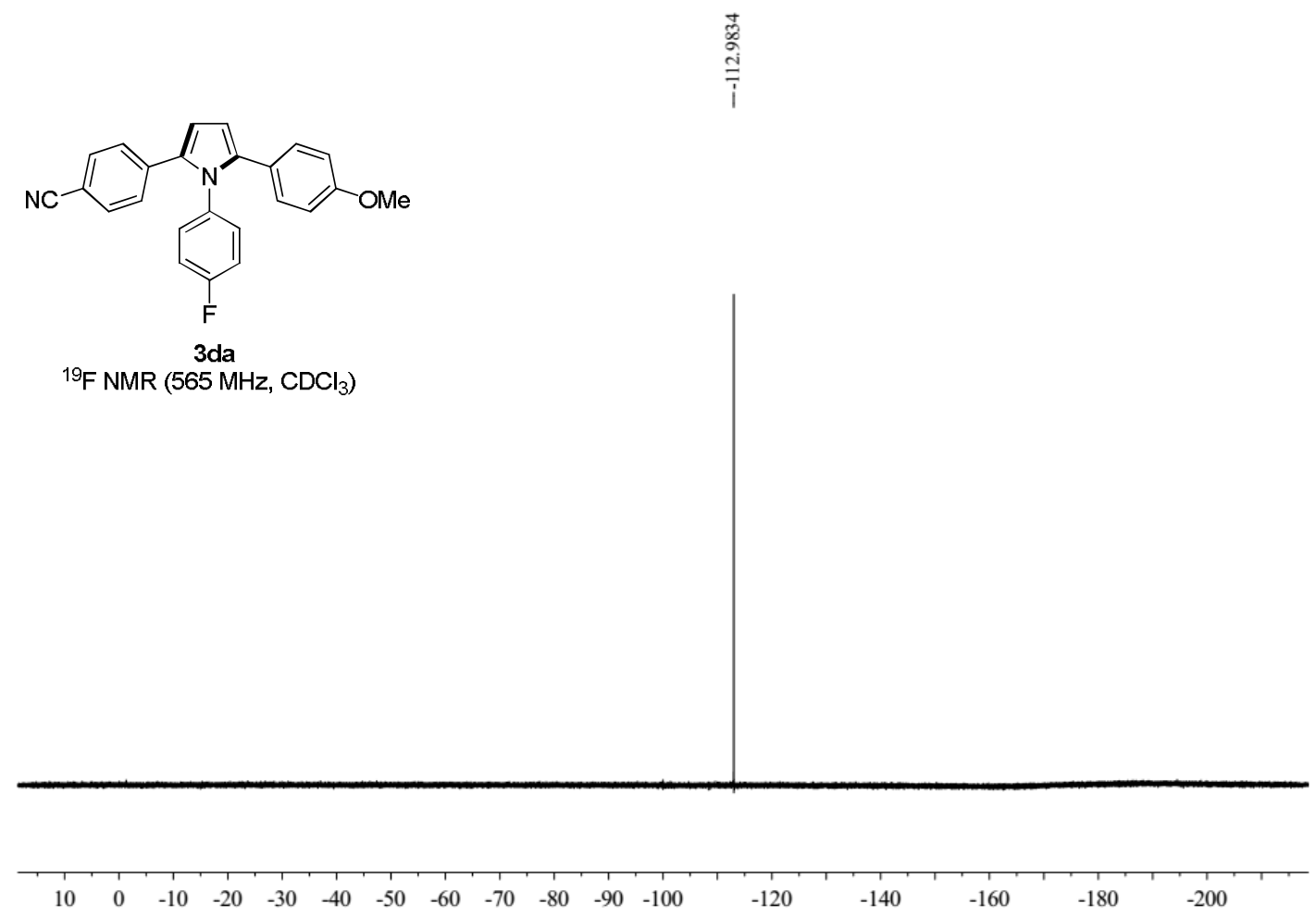

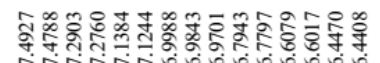

inivioiolibiob

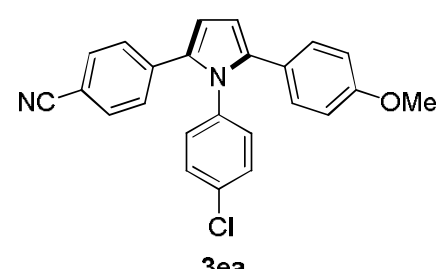

${ }^{1} \mathrm{H}$ NMR $\left(600 \mathrm{MHz}, \mathrm{CDCl}_{3}\right)$

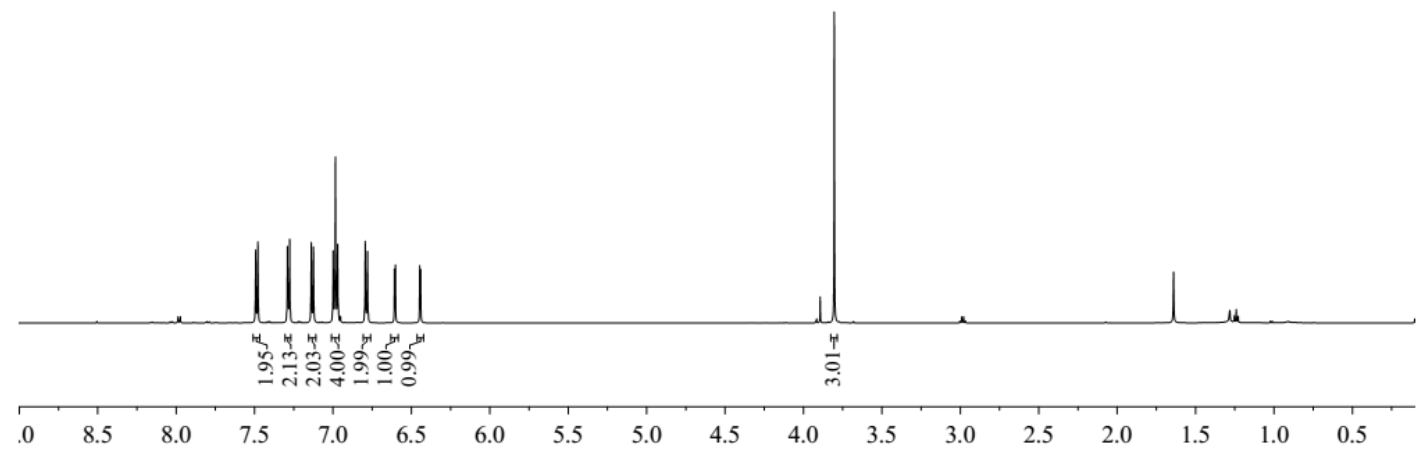




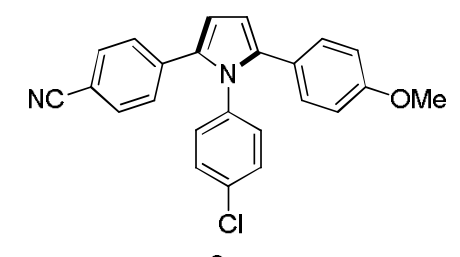

${ }^{13} \mathrm{C}$ NMR (151 MHz, $\left.\mathrm{CDCl}_{3}\right)$

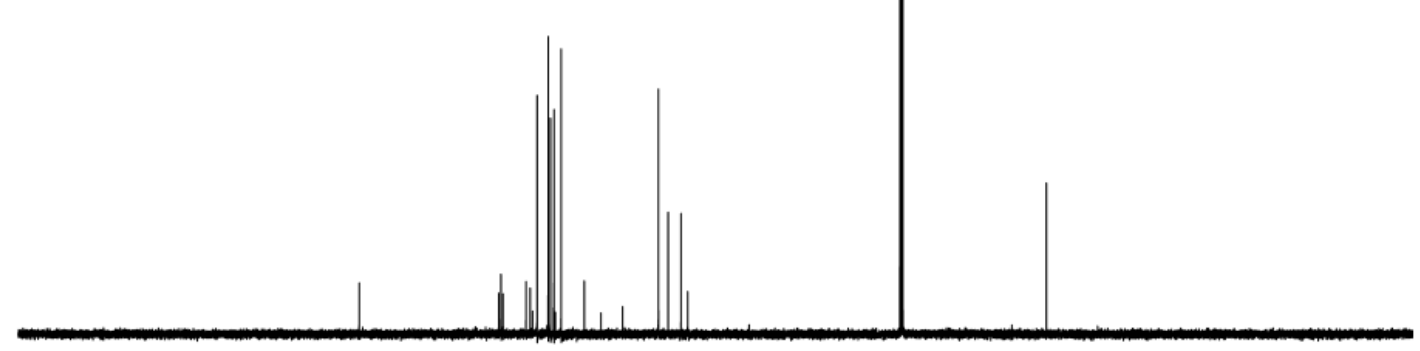

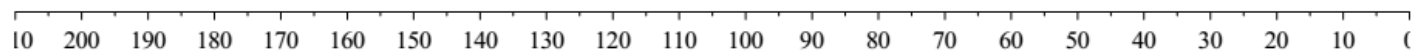

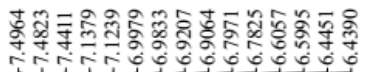

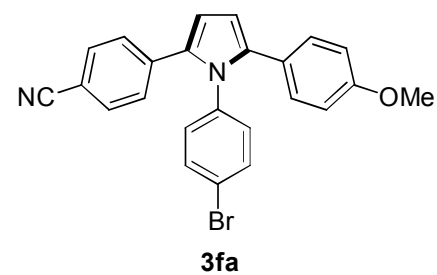

${ }^{1} \mathrm{H} \mathrm{NMR}\left(600 \mathrm{MHz}, \mathrm{CDCl}_{3}\right)$

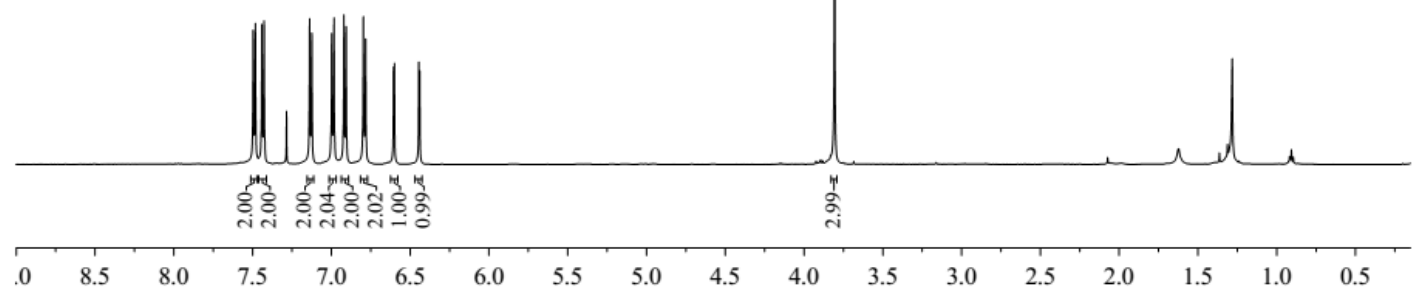




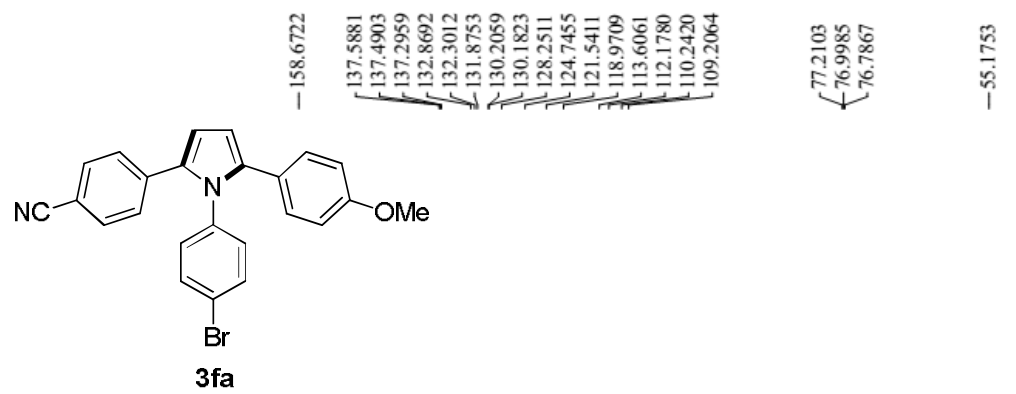

${ }^{13} \mathrm{C}$ NMR (151 MHz, $\mathrm{CDCl}$ )

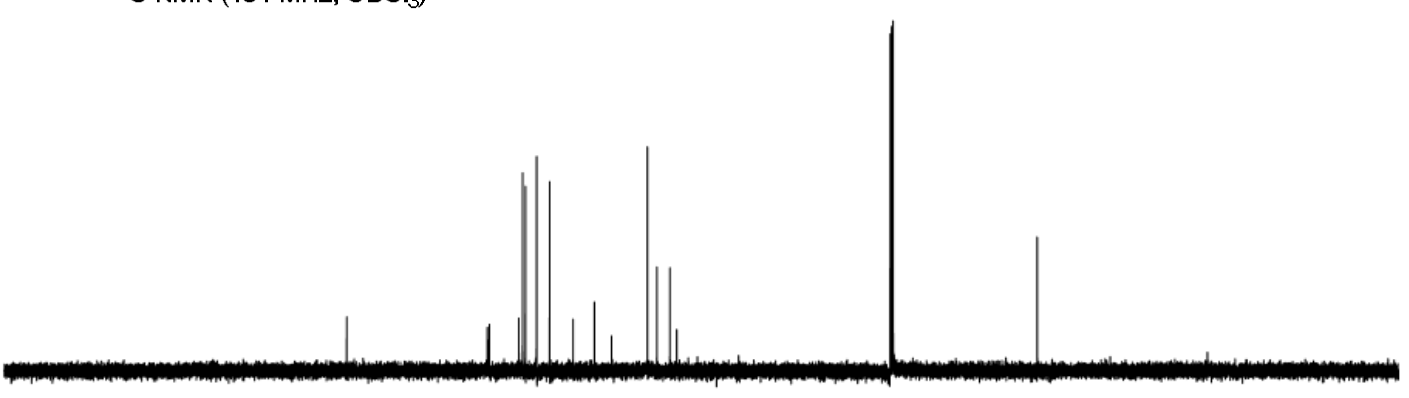

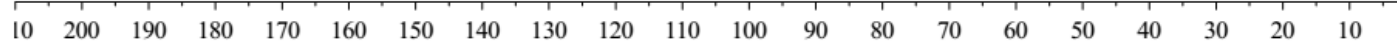

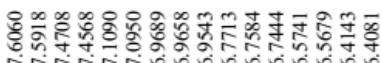

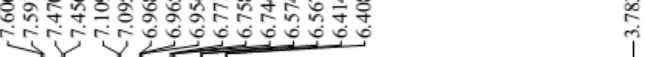

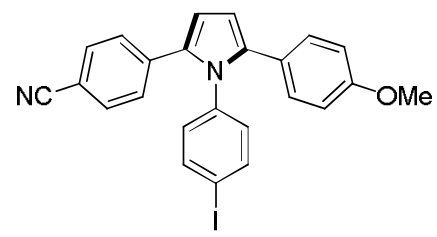

3ga

${ }^{1} \mathrm{H}$ NMR $\left(600 \mathrm{MHz}, \mathrm{CDCl}_{3}\right)$

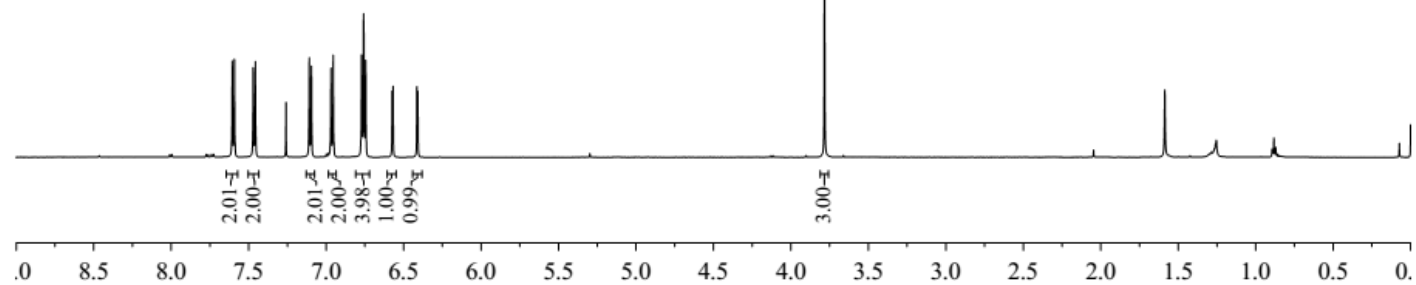




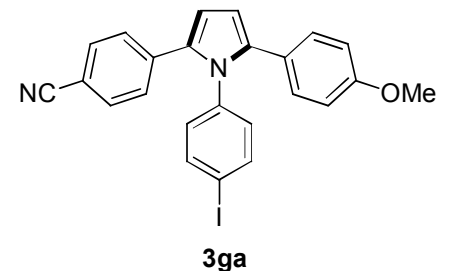

${ }^{13} \mathrm{C} \mathrm{NMR}\left(151 \mathrm{MHz}, \mathrm{CDCl}_{3}\right)$

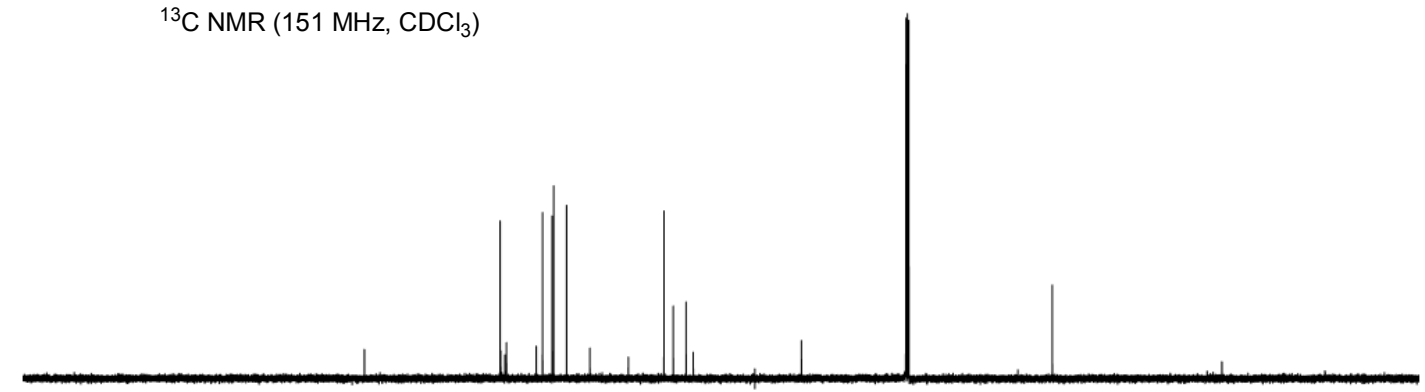

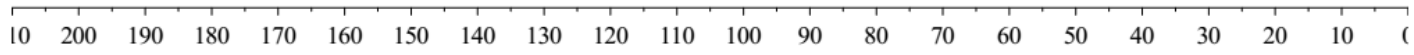

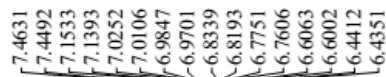<smiles>COc1ccc(-c2ccc(-c3ccc(C)cc3)n2-c2ccc(C#N)cc2)cc1</smiles>

${ }^{1} \mathrm{H}$ NMR $\left(600 \mathrm{MHz}, \mathrm{CDCl}_{3}\right)$

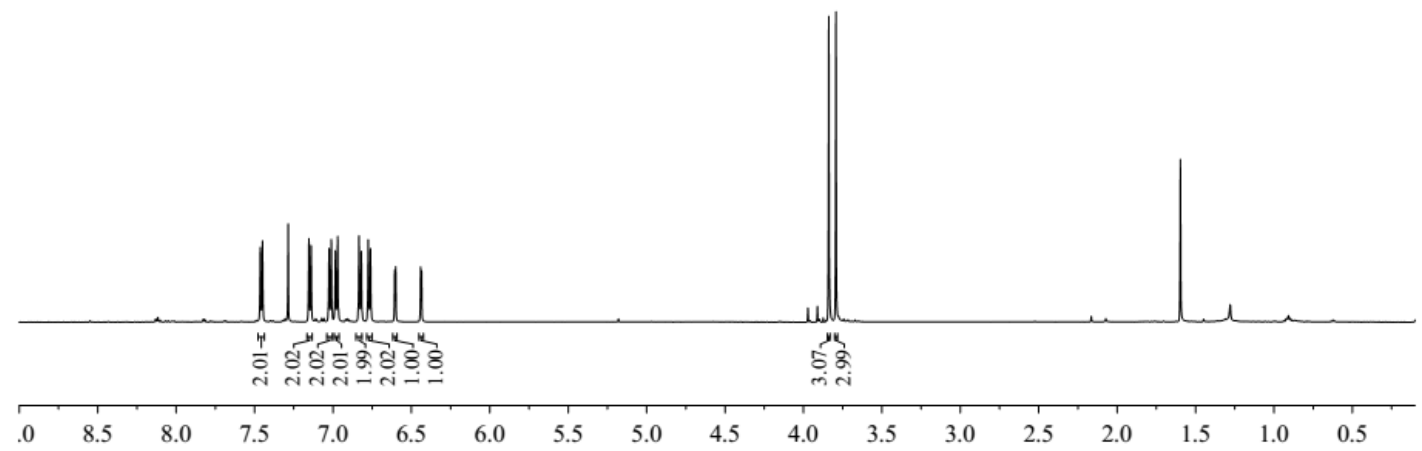



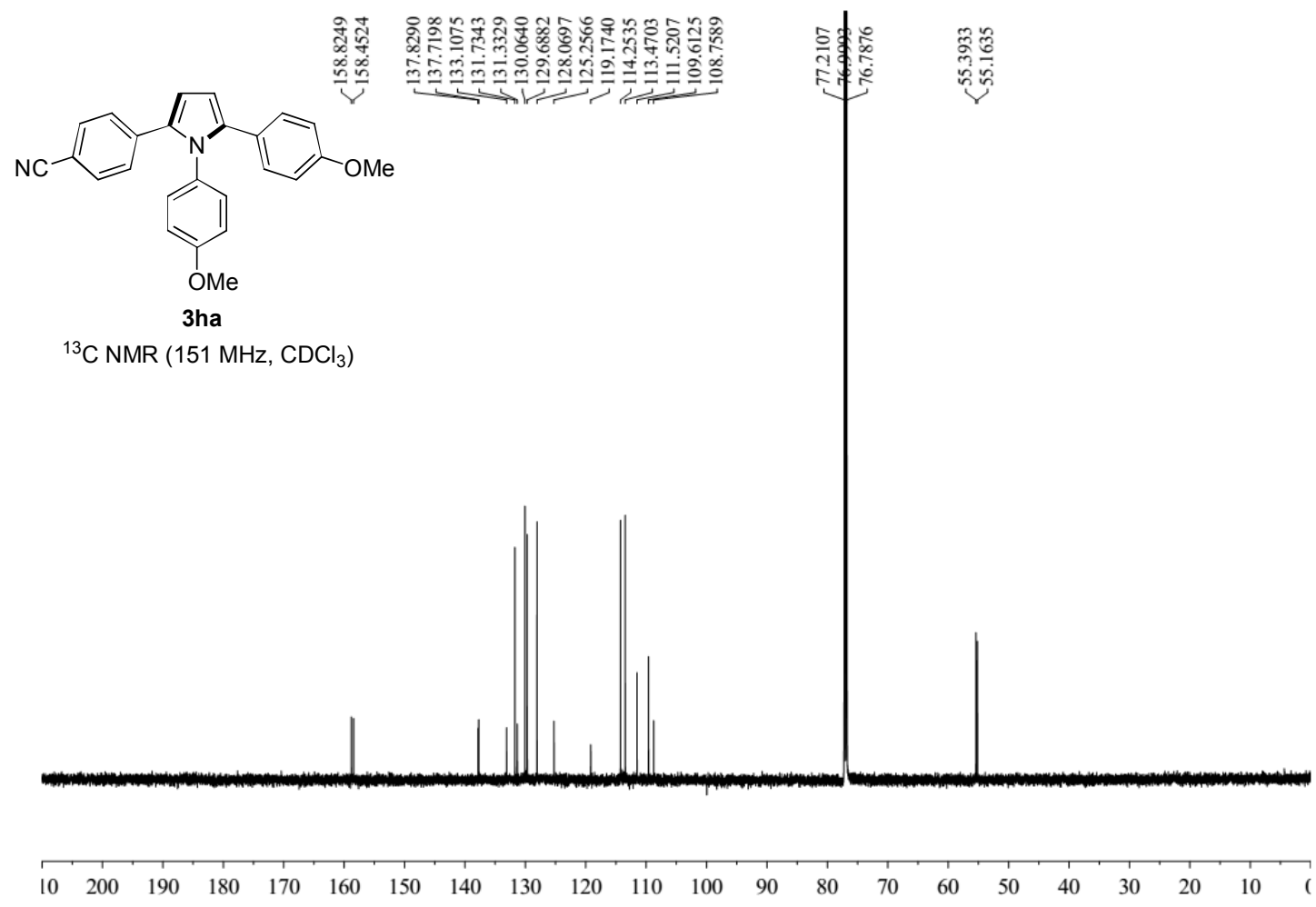

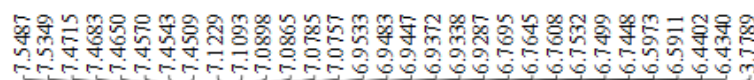

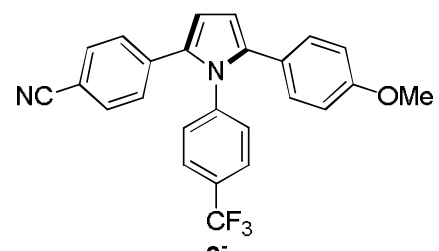

${ }^{1} \mathrm{H}$ NMR $\left(600 \mathrm{MHz}, \mathrm{CDCl}_{3}\right)$

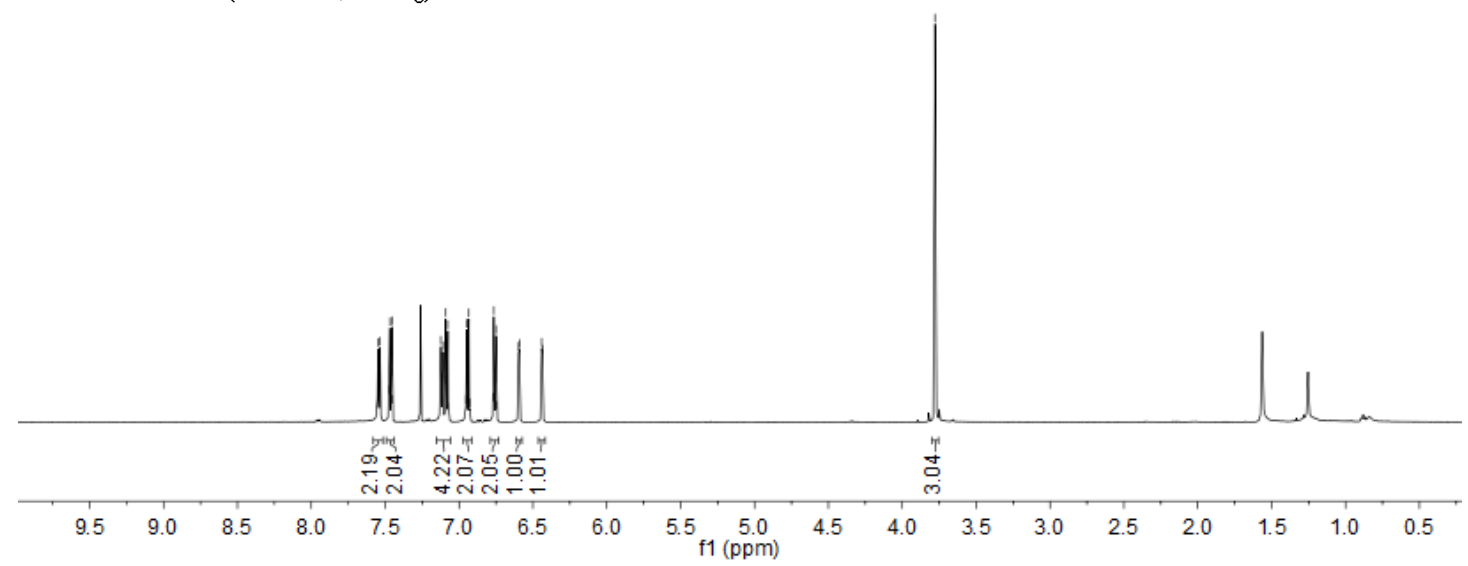




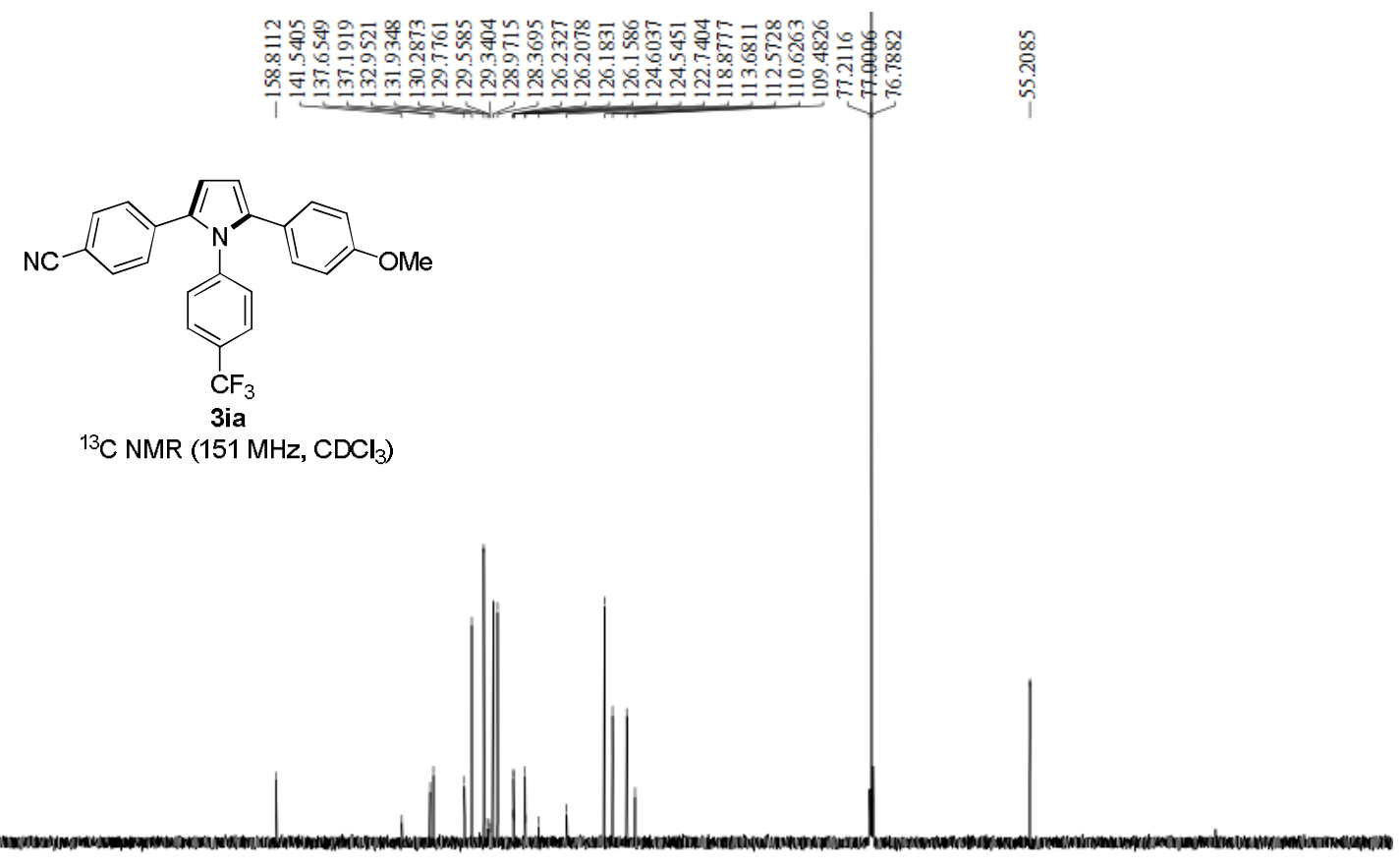

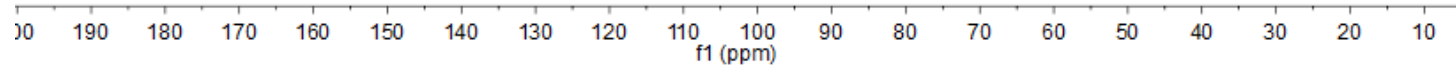

守<smiles>COc1ccc(-c2ccc(-c3ccc(C#N)cc3)n2-c2ccc(C(F)(F)F)cc2)cc1</smiles>

${ }^{19} \mathrm{~F} \mathrm{NMR}\left(565 \mathrm{MHz}, \mathrm{CDCl}_{3}\right)$

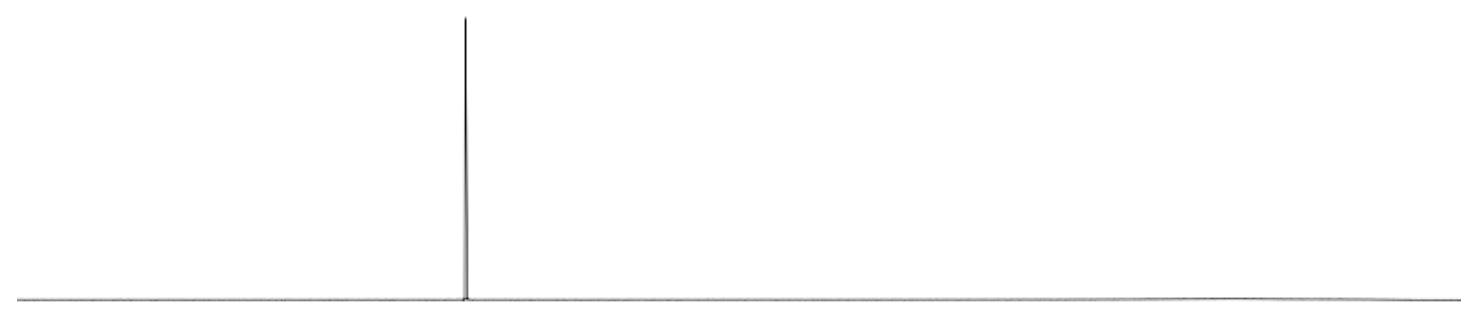

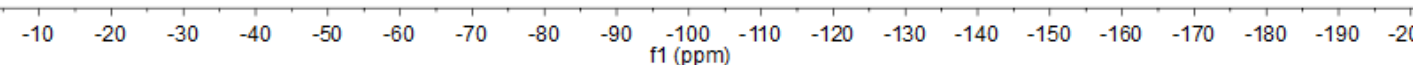




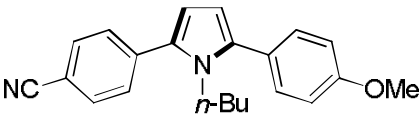

$3 \mathrm{ja}$

${ }^{1} \mathrm{H} \mathrm{NMR}\left(600 \mathrm{MHz}, \mathrm{CDCl}_{3}\right)$
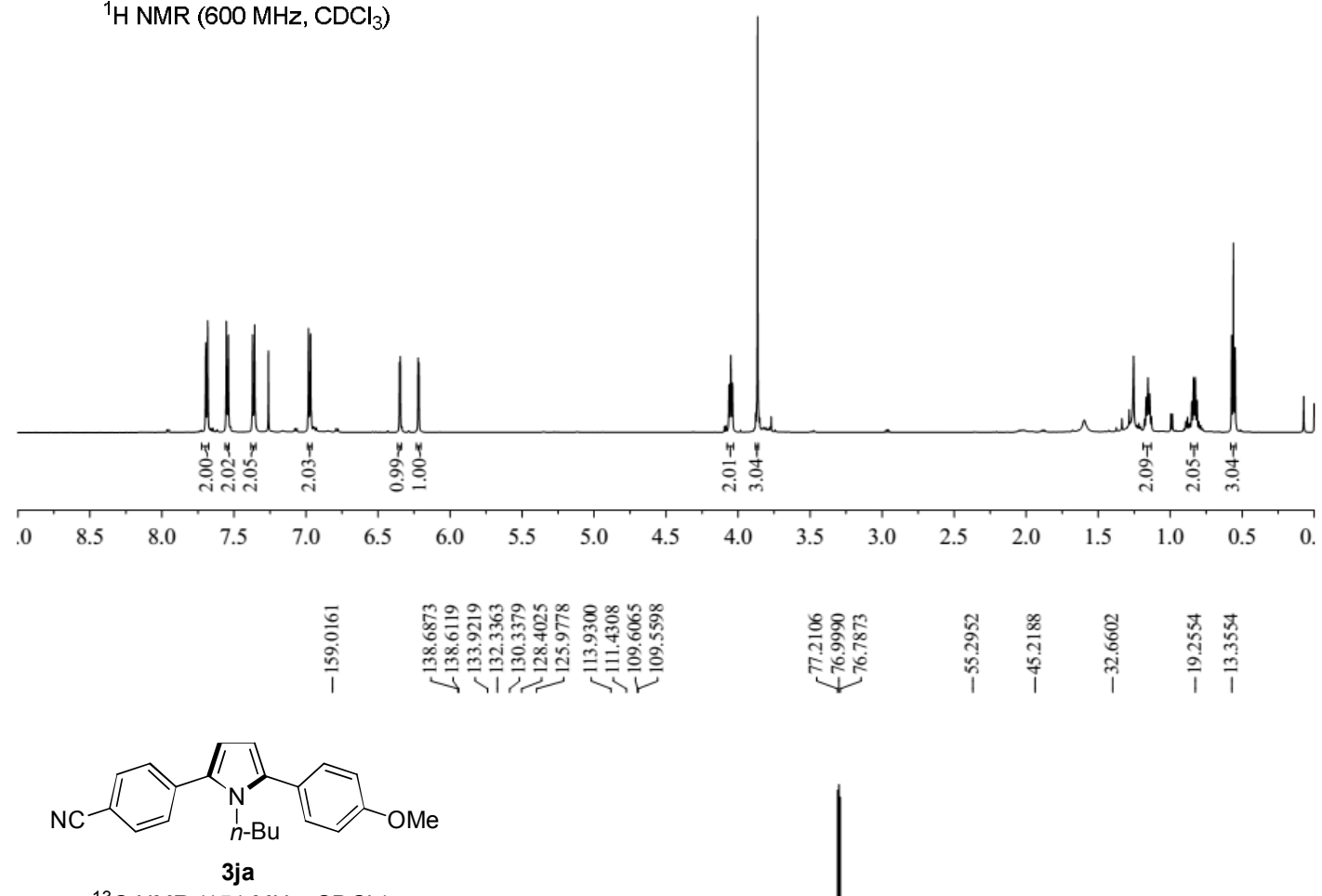

${ }^{13} \mathrm{C}$ NMR $\left(151 \mathrm{MHz}, \mathrm{CDCl}_{3}\right)$

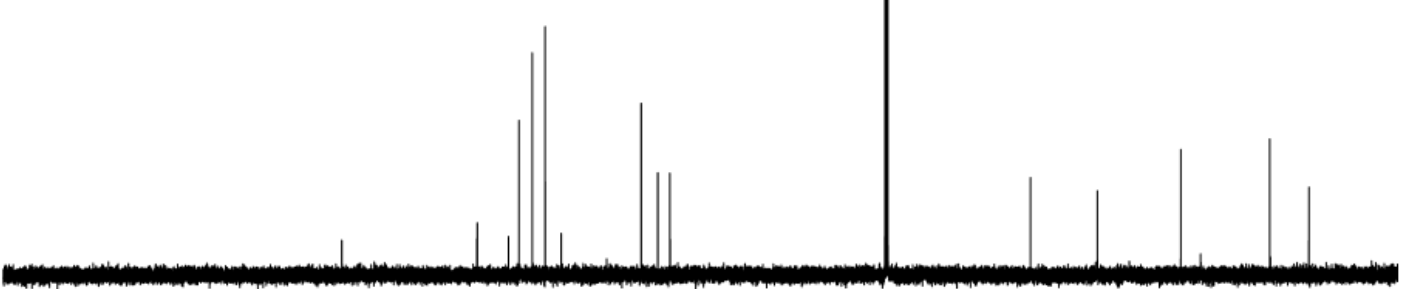

$\begin{array}{lllllllllllllllllllll}10 & 200 & 190 & 180 & 170 & 160 & 150 & 140 & 130 & 120 & 110 & 100 & 90 & 80 & 70 & 60 & 50 & 40 & 30 & 20 & 10\end{array}$ 

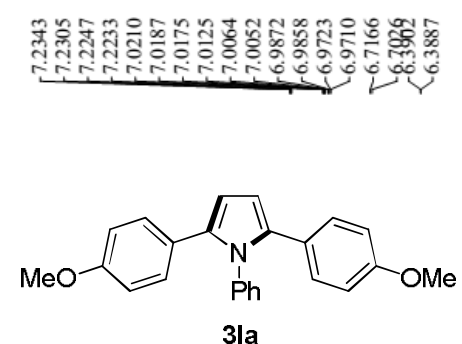

${ }^{1} \mathrm{H} \mathrm{NMR}\left(600 \mathrm{MHz}, \mathrm{CDCl}_{3}\right)$

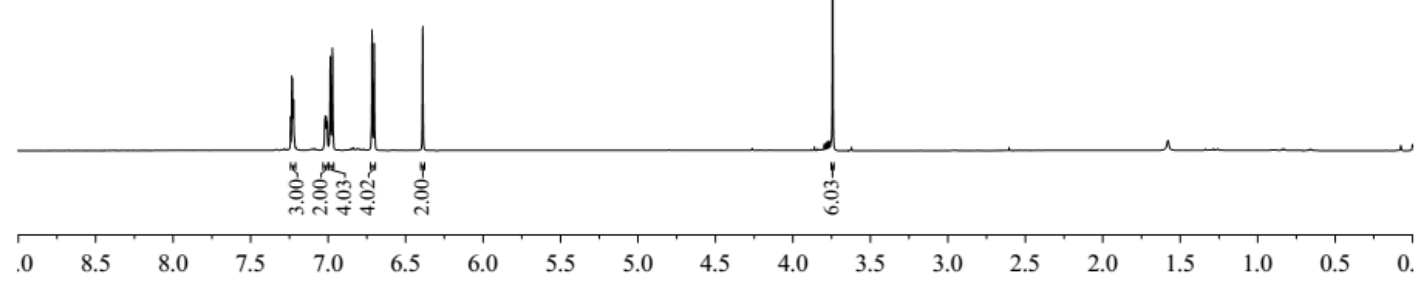

\begin{tabular}{|c|c|c|}
\hline 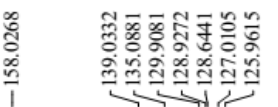 & 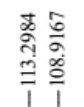 & 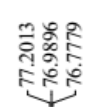 \\
\hline
\end{tabular}

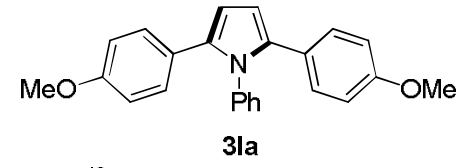

${ }^{13} \mathrm{C}$ NMR $\left(151 \mathrm{MHz}, \mathrm{CDCl}_{3}\right)$

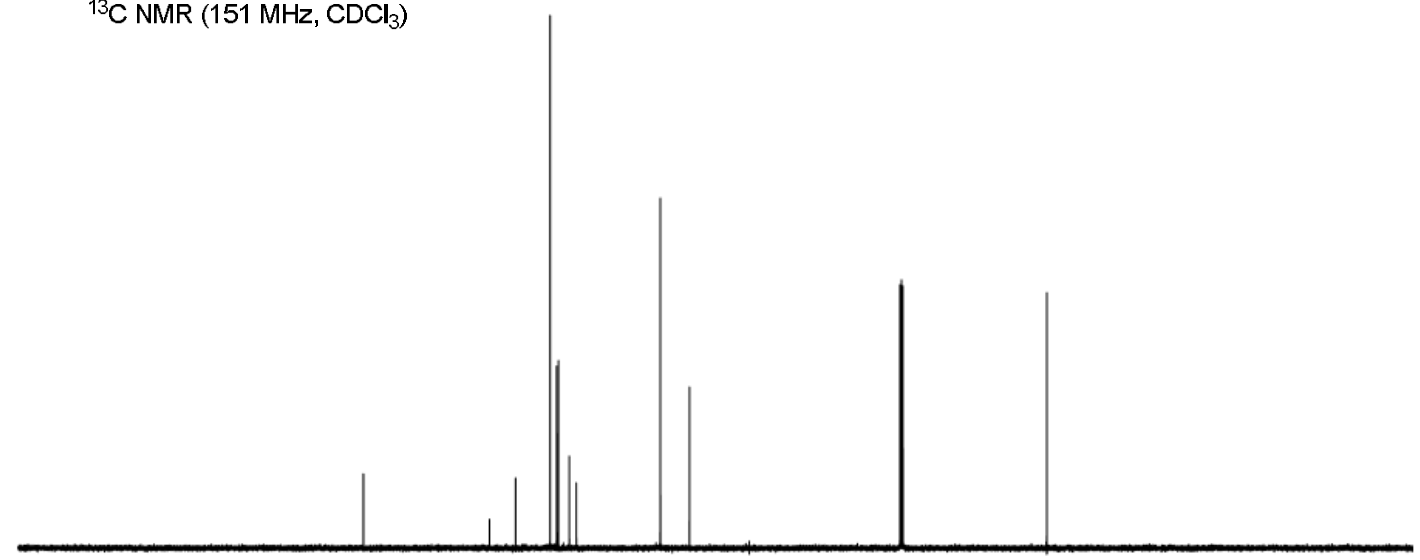

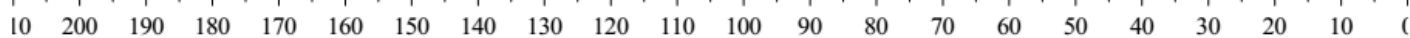




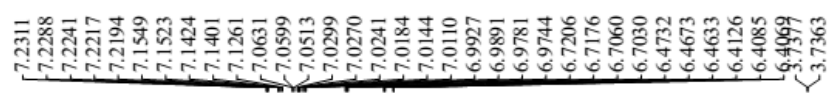

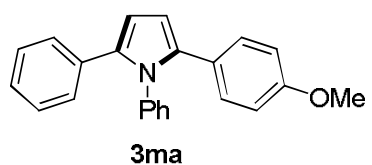

${ }^{1} \mathrm{H}$ NMR $\left(600 \mathrm{MHz}, \mathrm{CDCl}_{3}\right)$
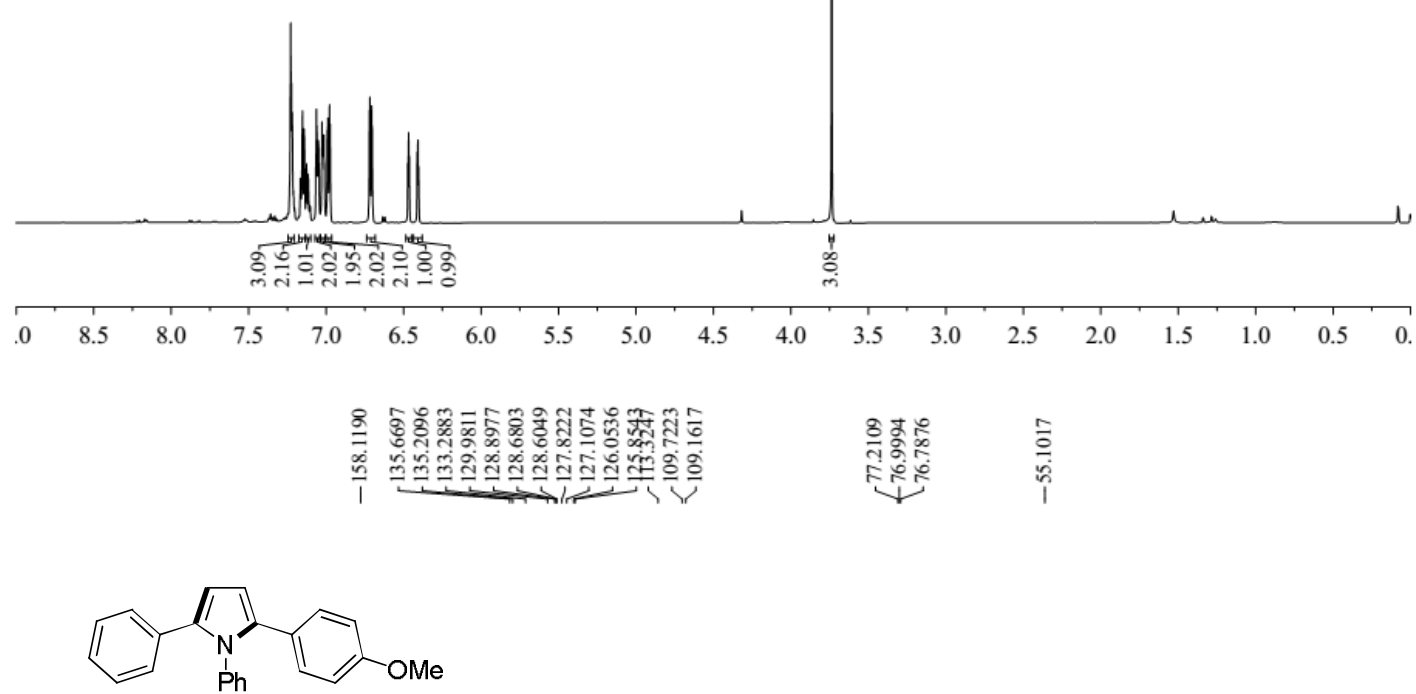

$3 \mathrm{ma}$

${ }^{13} \mathrm{C}$ NMR $\left(151 \mathrm{MHz}, \mathrm{CDCl}_{3}\right)$

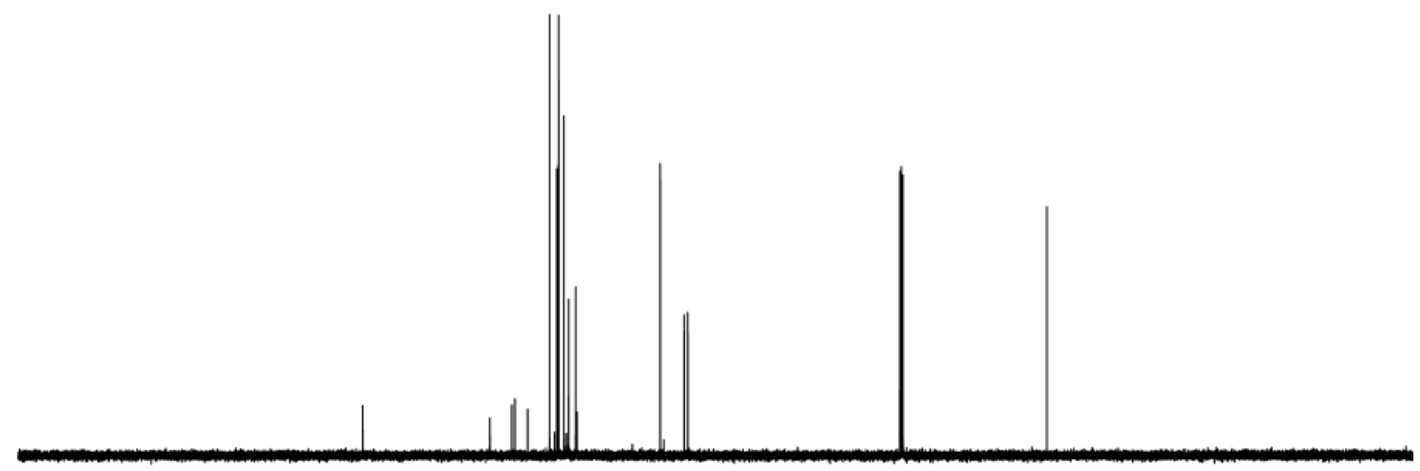

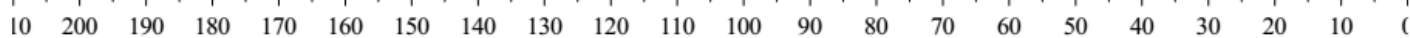




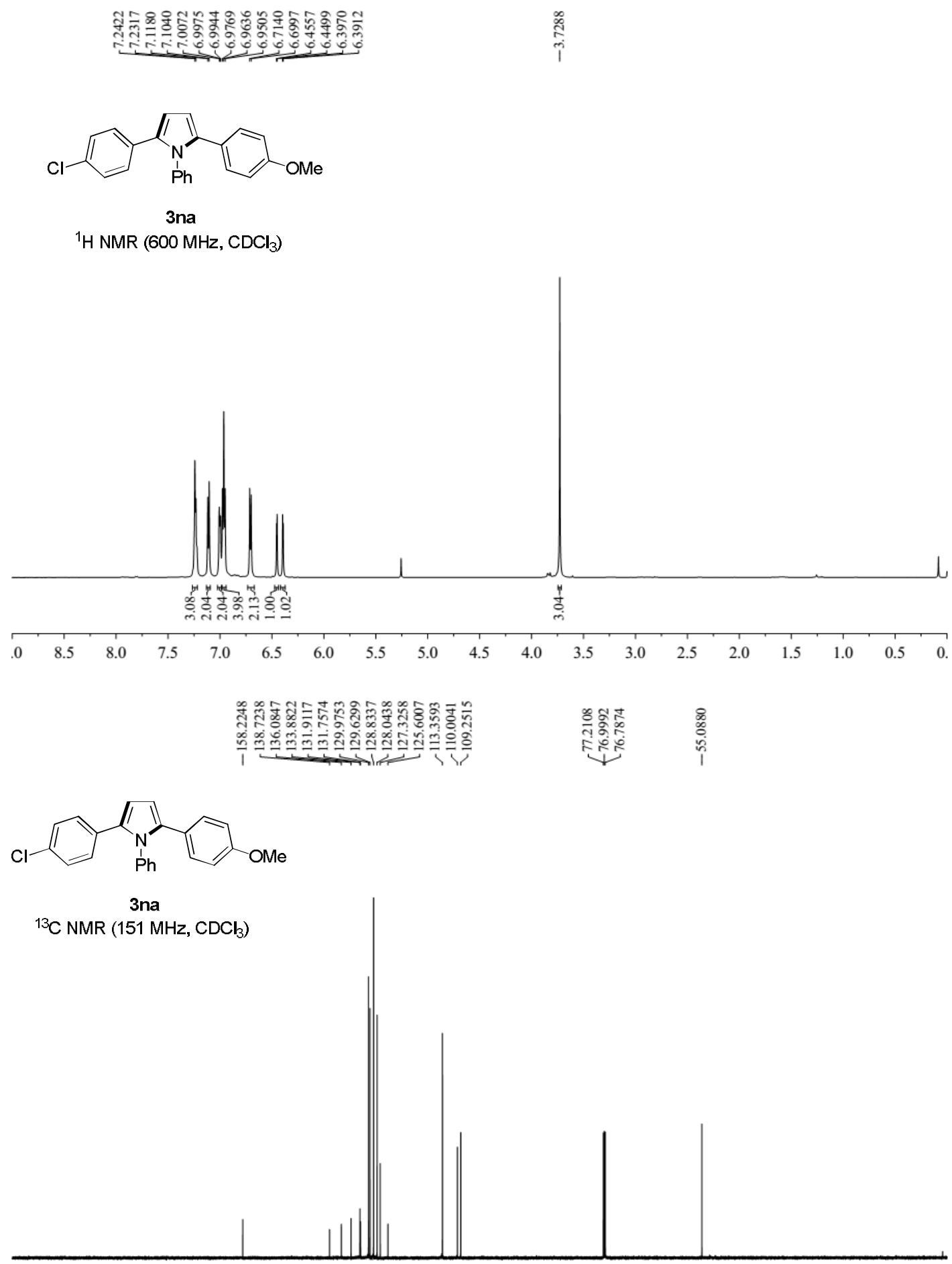

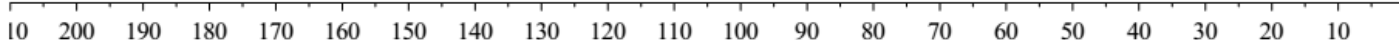




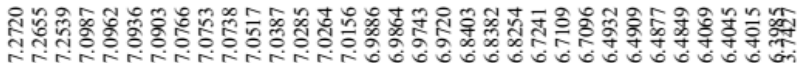

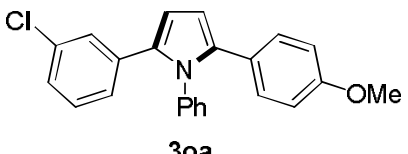

${ }^{1} \mathrm{H}$ NMR $\left(600 \mathrm{MHz}, \mathrm{CDCl}_{3}\right)$

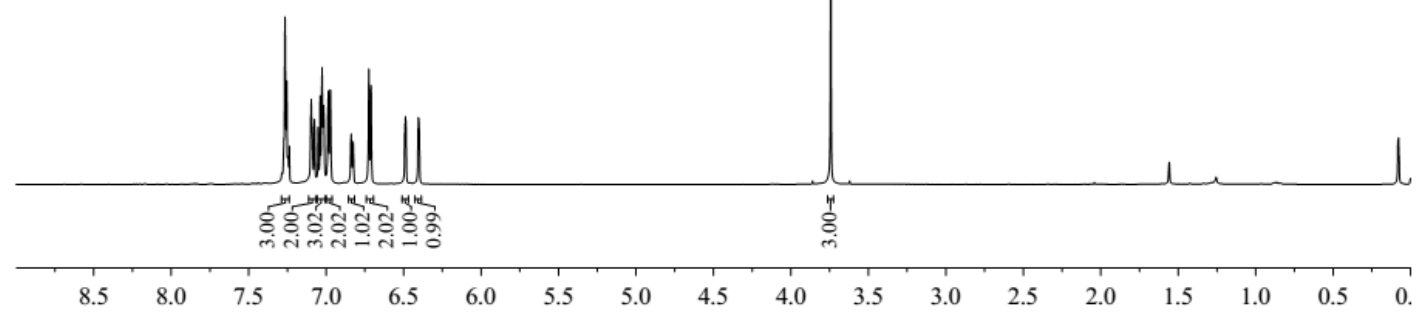

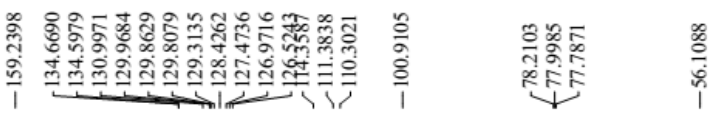

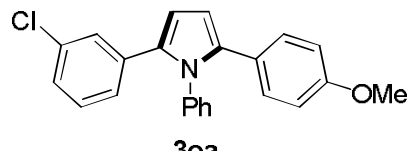

${ }^{13} \mathrm{C}$ NMR (151 MHz, $\left.\mathrm{CDCl}_{3}\right)$

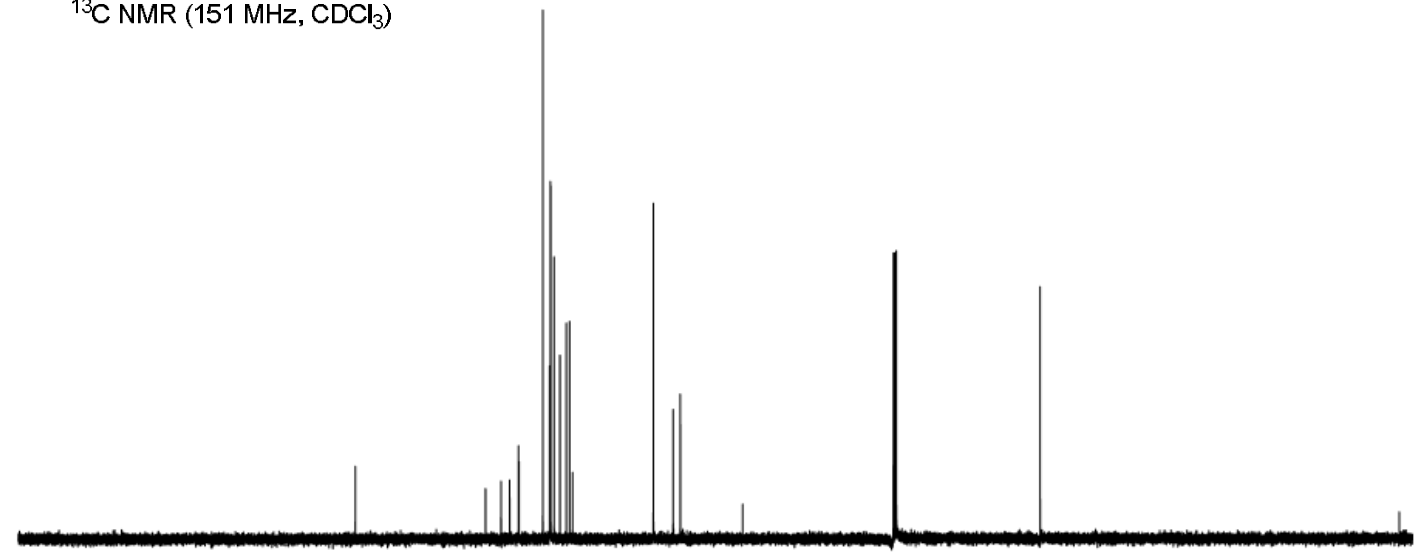

$\begin{array}{lllllllllllllllllllll}10 & 200 & 190 & 180 & 170 & 160 & 150 & 140 & 130 & 120 & 110 & 100 & 90 & 80 & 70 & 60 & 50 & 40 & 30 & 20 & 10\end{array}$ 


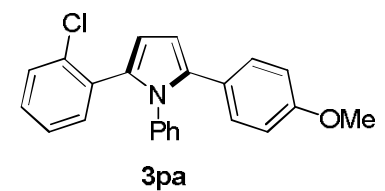

${ }^{1} \mathrm{H}$ NMR $\left(600 \mathrm{MHz}, \mathrm{CDCl}_{3}\right)$
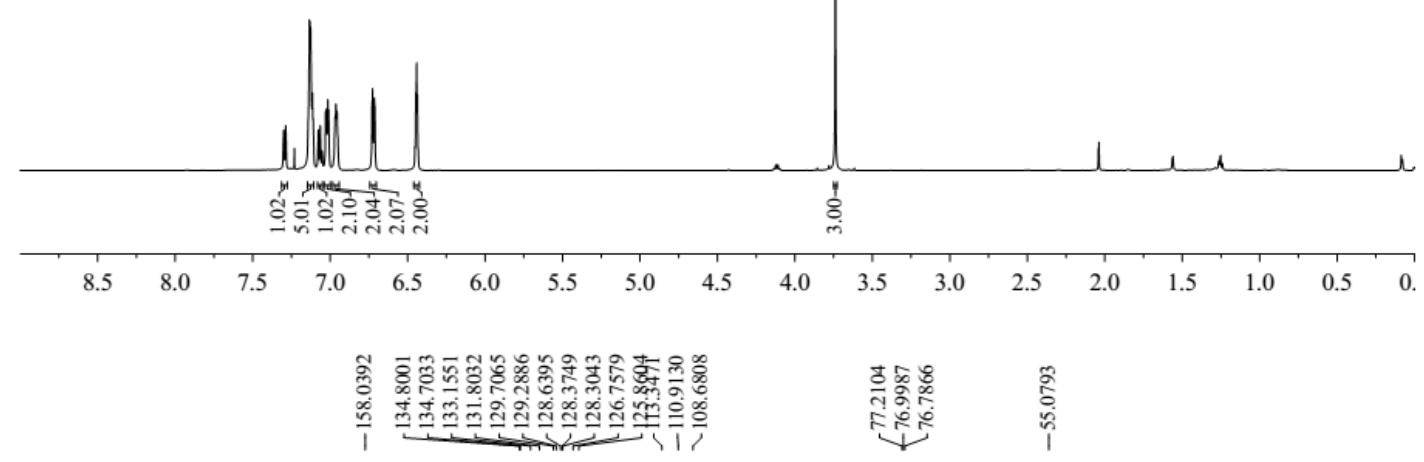

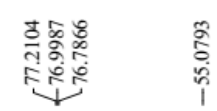<smiles>COc1ccc(-c2ccc(-c3ccccc3Cl)n2-c2ccccc2)cc1</smiles>

3pa

$\left.{ }^{13} \mathrm{C} \mathrm{NMR} \mathrm{(151} \mathrm{MHz,} \mathrm{CDCl}_{3}\right)$

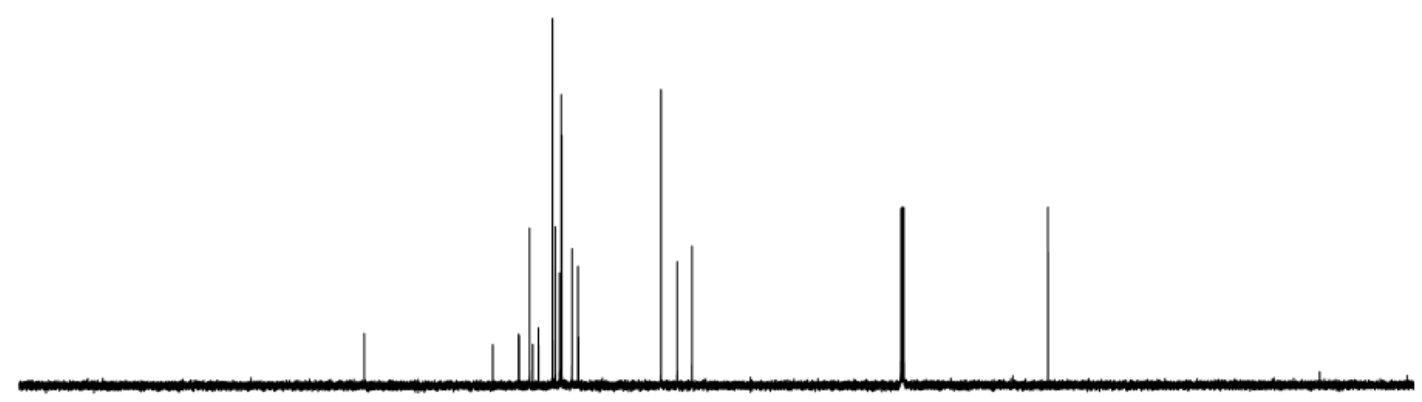

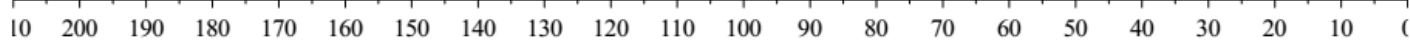




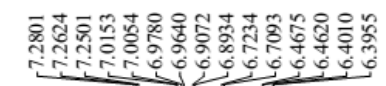

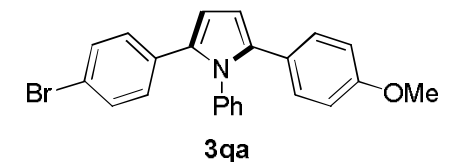

${ }^{1} \mathrm{H}$ NMR $\left(600 \mathrm{MHz}, \mathrm{CDCl}_{3}\right)$
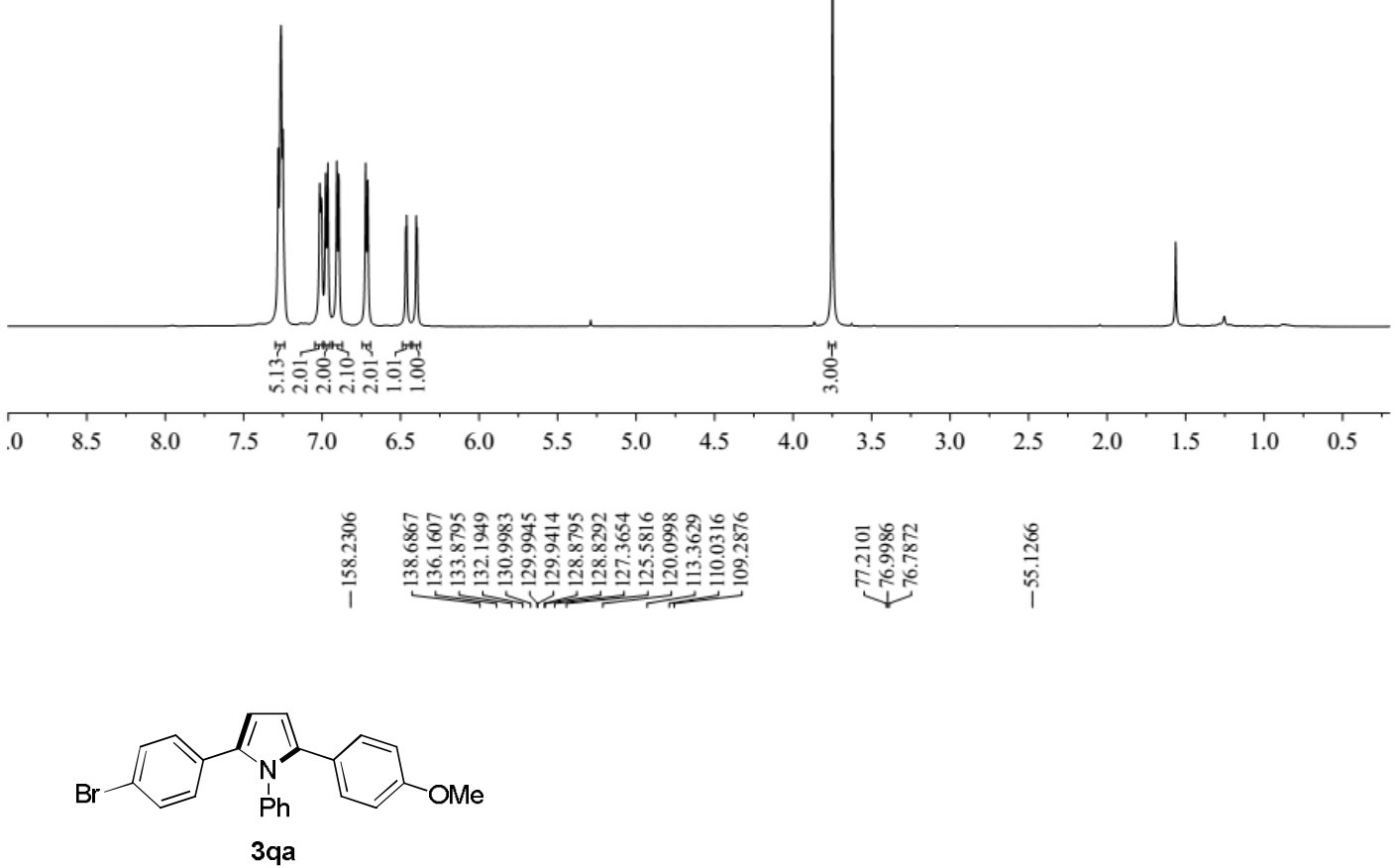

${ }^{13} \mathrm{C}$ NMR (151 MHz, $\mathrm{CDCl}_{3}$ )

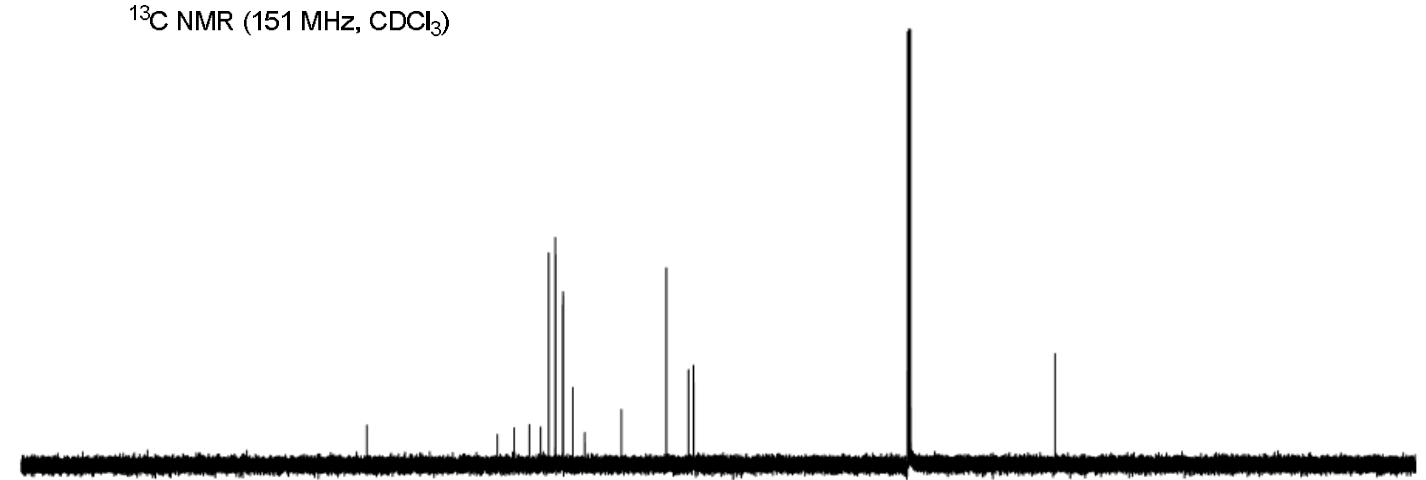

$\begin{array}{lllllllllllllllllllll}10 & 200 & 190 & 180 & 170 & 160 & 150 & 140 & 130 & 120 & 110 & 100 & 90 & 80 & 70 & 60 & 50 & 40 & 30 & 20 & 10\end{array}$ 


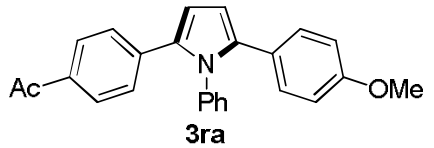

${ }^{1} \mathrm{H}$ NMR $\left(600 \mathrm{MHz}, \mathrm{CDCl}_{3}\right)$
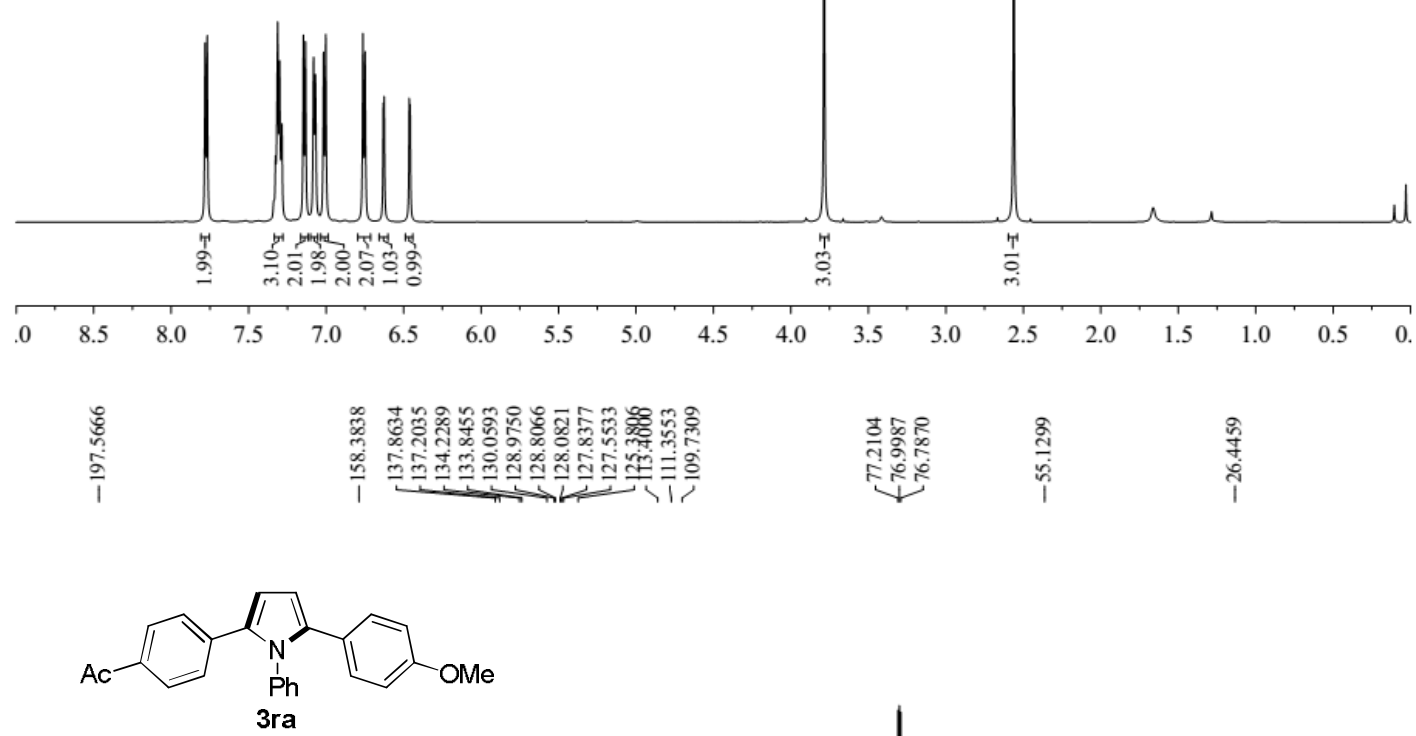

${ }^{13} \mathrm{C}$ NMR (151 MHz, $\left.\mathrm{CDCl}_{3}\right)$

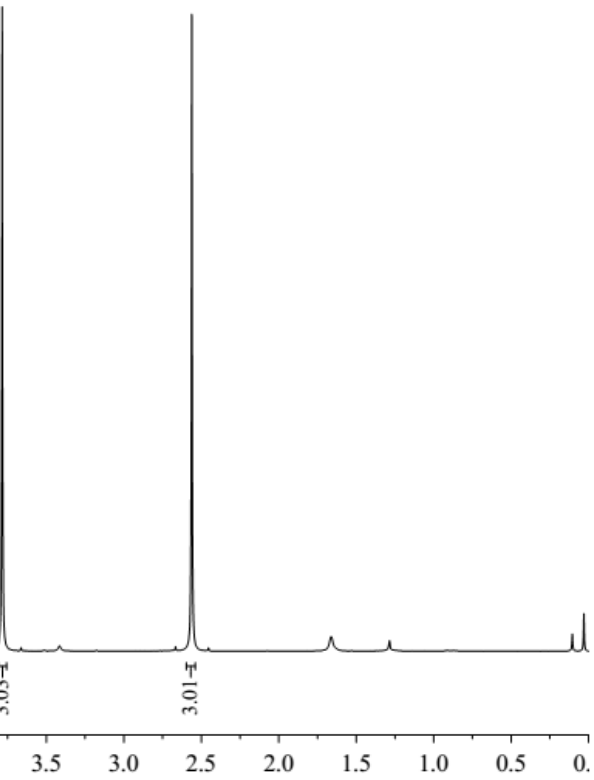

旁

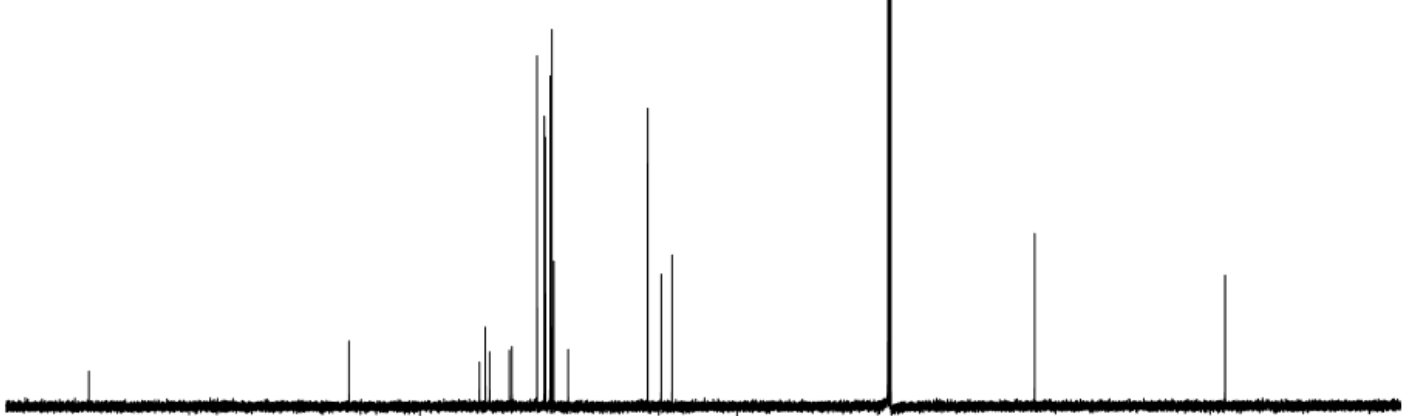

$\begin{array}{lllllllllllllllllllll}10 & 200 & 190 & 180 & 170 & 160 & 150 & 140 & 130 & 120 & 110 & 100 & 90 & 80 & 70 & 60 & 50 & 40 & 30 & 20 & 10\end{array}$ 


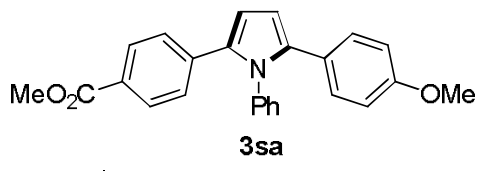

${ }^{1} \mathrm{H}$ NMR $\left(600 \mathrm{MHz}, \mathrm{CDCl}_{3}\right)$
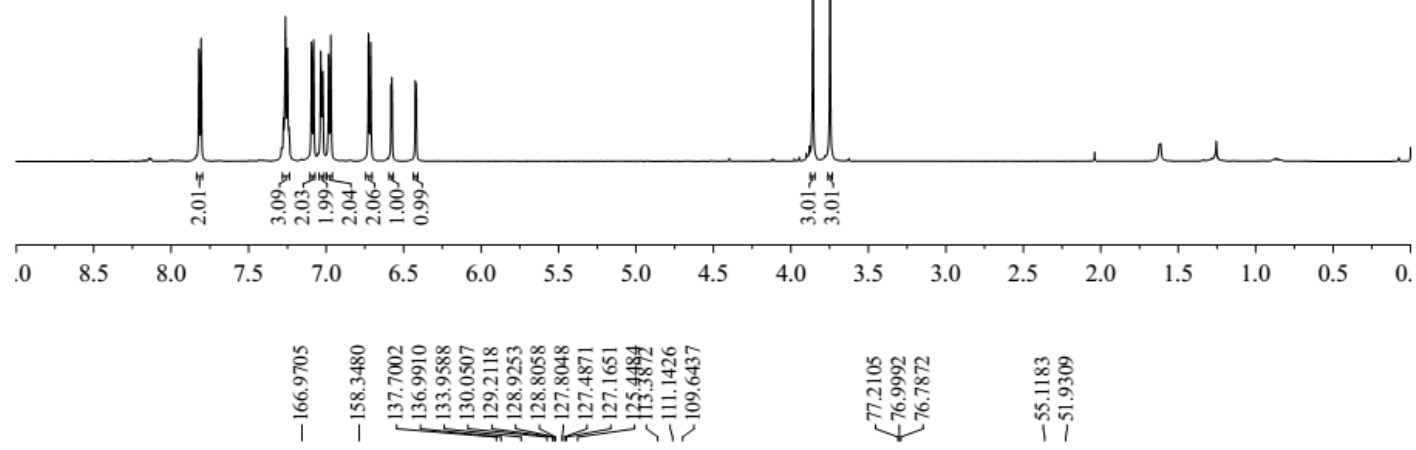

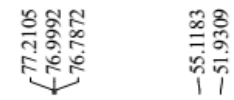

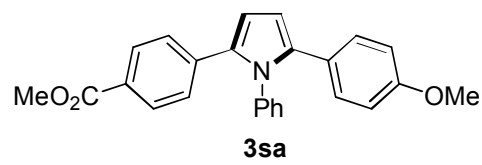

${ }^{13} \mathrm{C}$ NMR $\left(151 \mathrm{MHz}, \mathrm{CDCl}_{3}\right)$

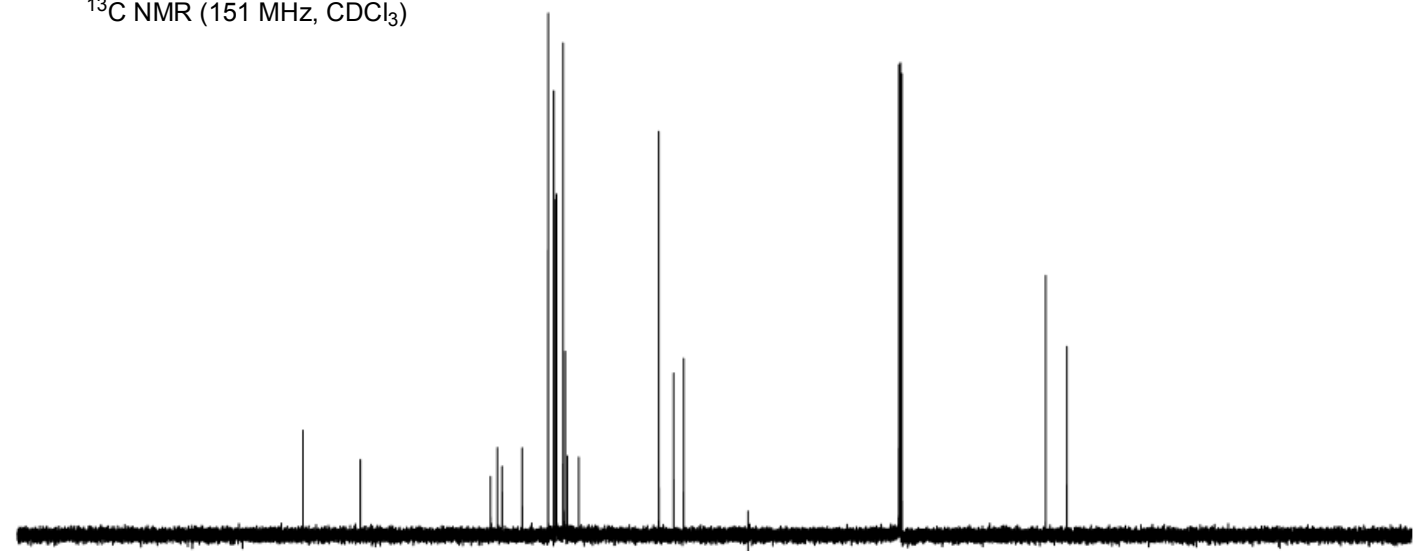

$\begin{array}{lllllllllllllllllllll}10 & 200 & 190 & 180 & 170 & 160 & 150 & 140 & 130 & 120 & 110 & 100 & 90 & 80 & 70 & 60 & 50 & 40 & 30 & 20 & 10\end{array}$ 


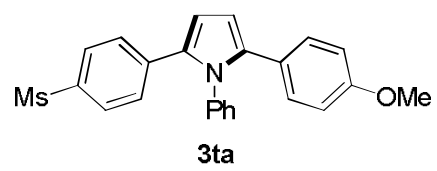

${ }^{1} \mathrm{H}$ NMR $\left(600 \mathrm{MHz}, \mathrm{CDCl}_{3}\right)$
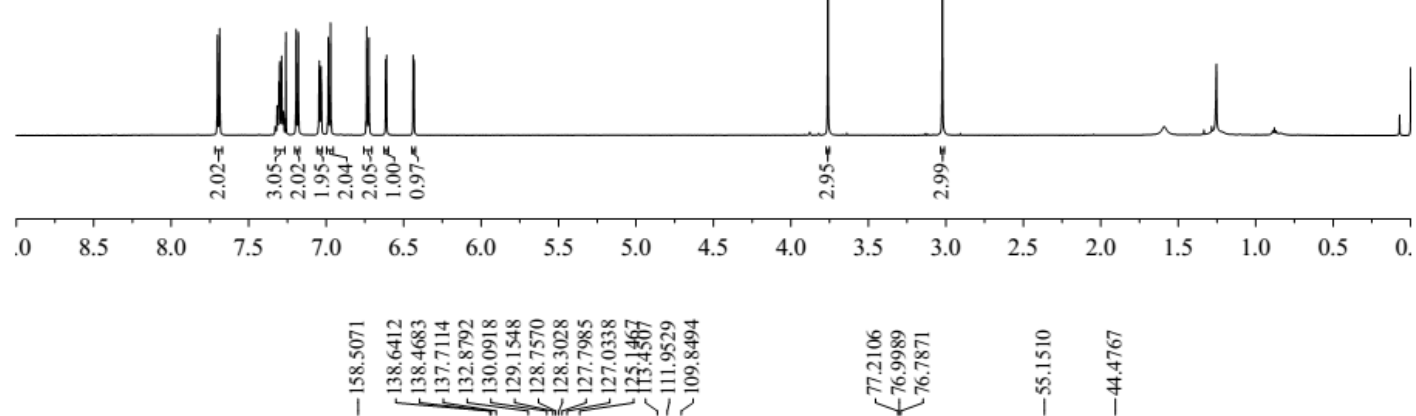

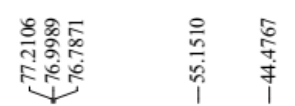<smiles>COc1ccc(-c2ccc(-c3ccc(C)cc3)n2-c2ccccc2)cc1</smiles>

${ }^{13} \mathrm{C}$ NMR (151 MHz, $\left.\mathrm{CDCl} 3\right)$

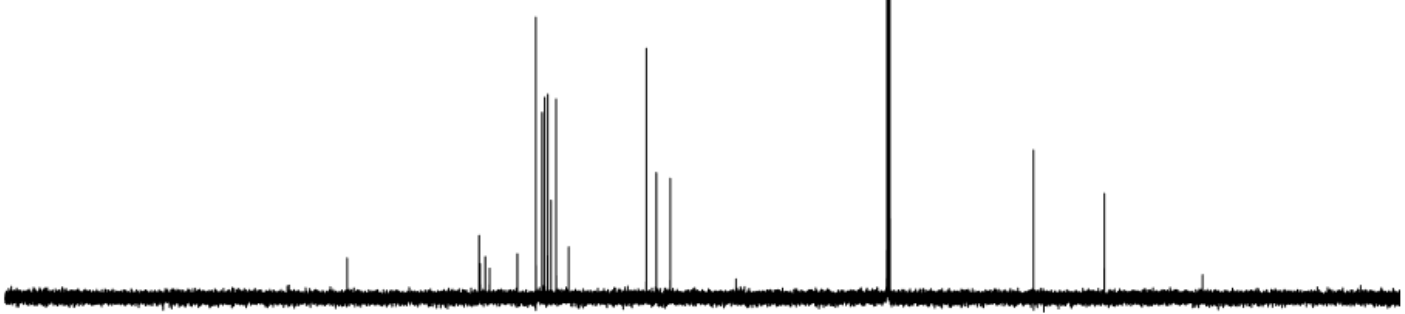

$\begin{array}{lllllllllllllllllllll}10 & 200 & 190 & 180 & 170 & 160 & 150 & 140 & 130 & 120 & 110 & 100 & 90 & 80 & 70 & 60 & 50 & 40 & 30 & 20 & 10\end{array}$ 


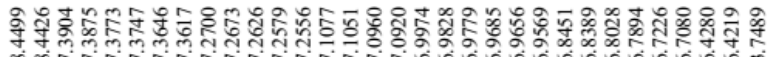

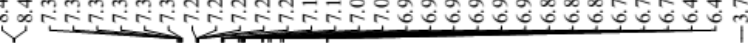

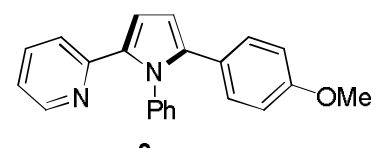

${ }^{1} \mathrm{H} \mathrm{NMR}\left(600 \mathrm{MHz}, \mathrm{CDCl}_{3}\right)$
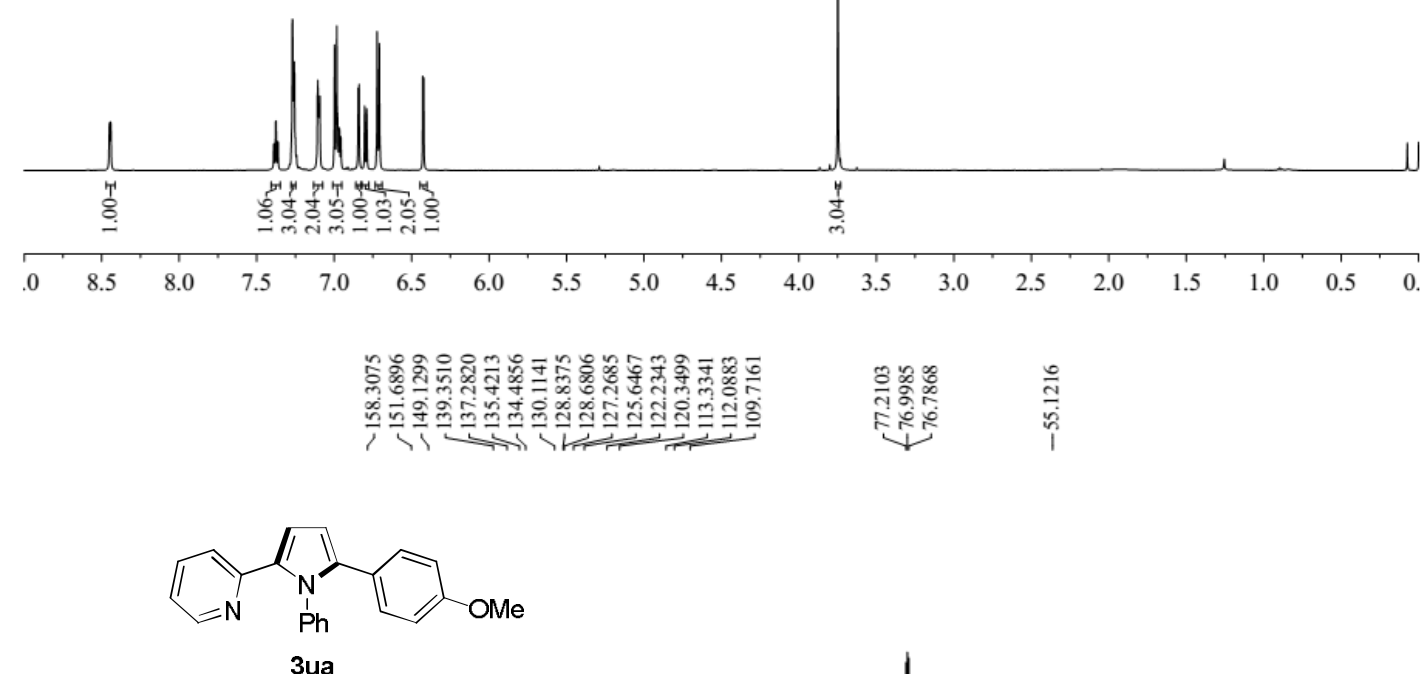

$\left.{ }^{13} \mathrm{C} \mathrm{NMR} \mathrm{(151} \mathrm{MHz,} \mathrm{CDCl}\right)$

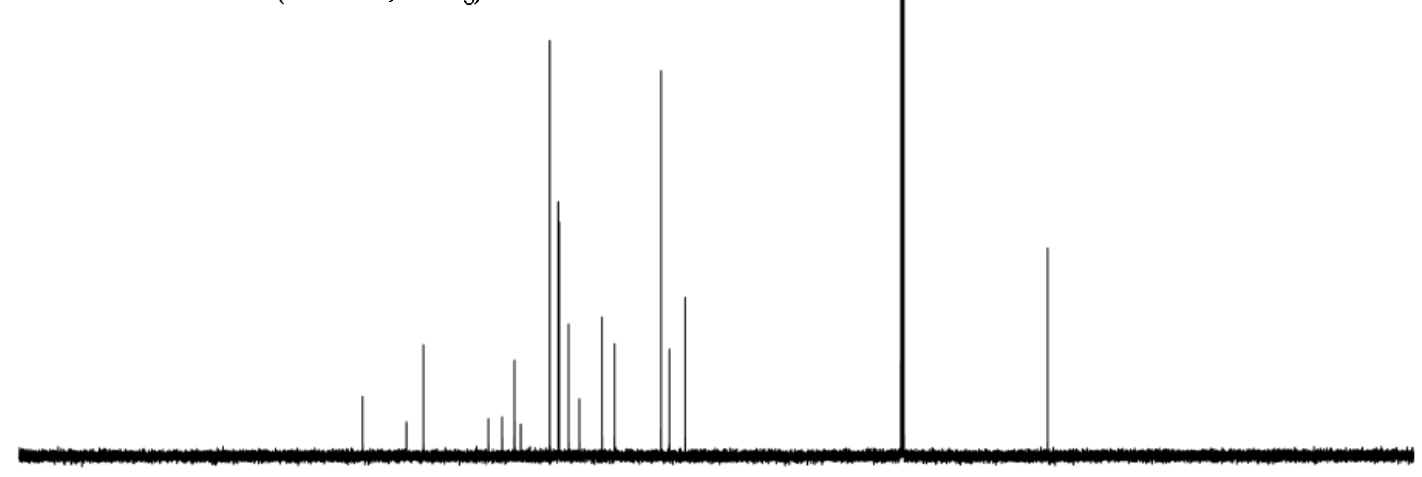

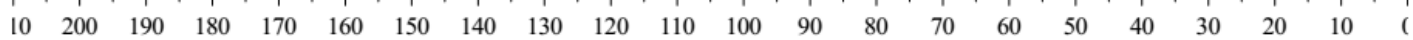




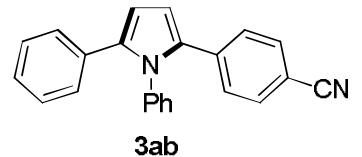

${ }^{1} \mathrm{H}$ NMR $\left(600 \mathrm{MHz}, \mathrm{CDCl}_{3}\right)$
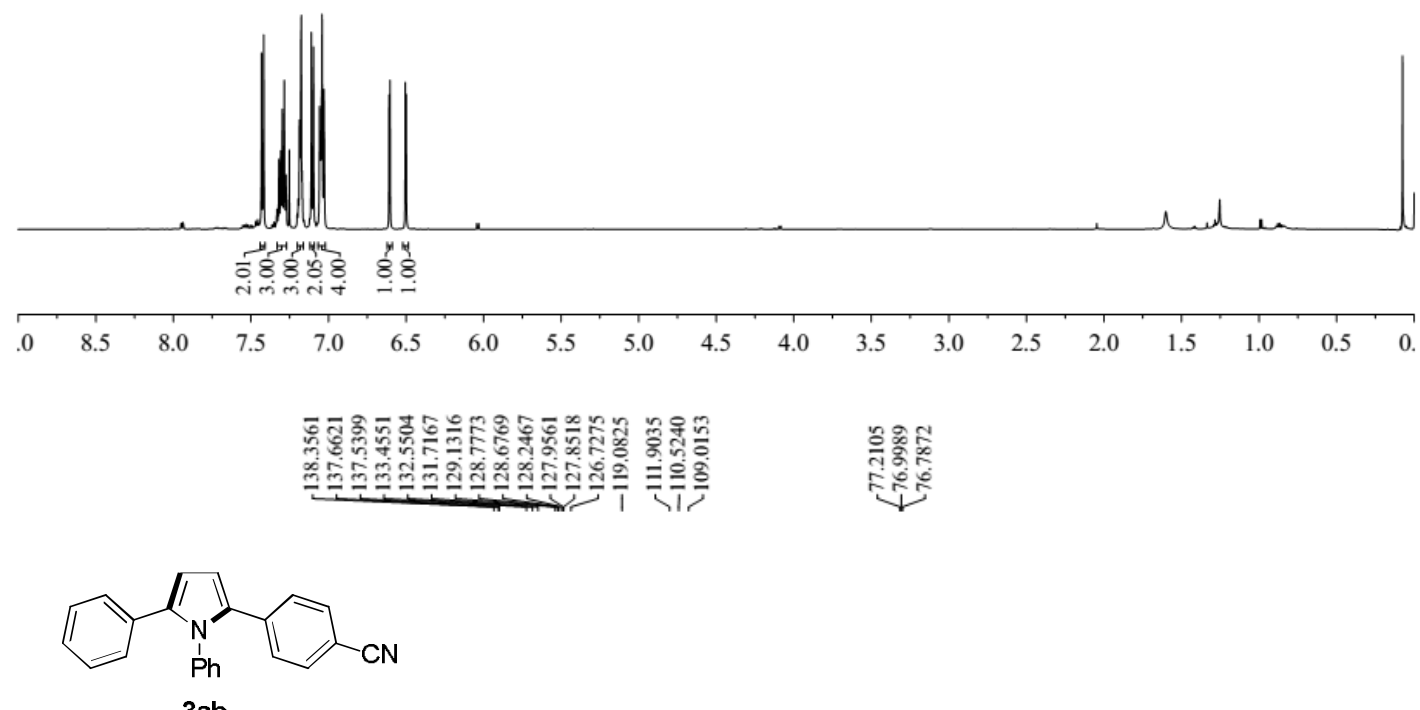

$3 a b$

${ }^{13} \mathrm{C} \mathrm{NMR}\left(151 \mathrm{MHz}, \mathrm{CDCl}_{3}\right)$

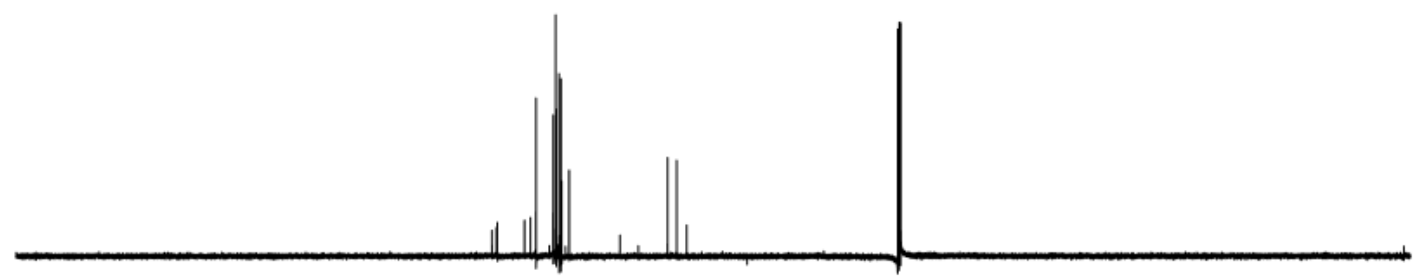

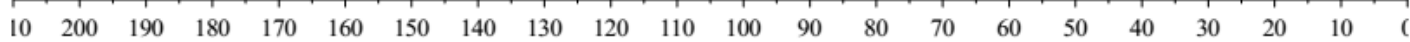




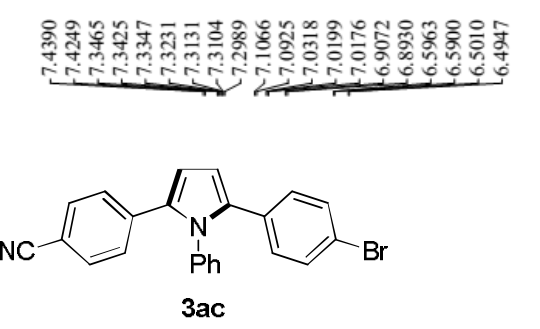

${ }^{1} \mathrm{H}$ NMR $\left(600 \mathrm{MHz}, \mathrm{CDCl}_{3}\right)$

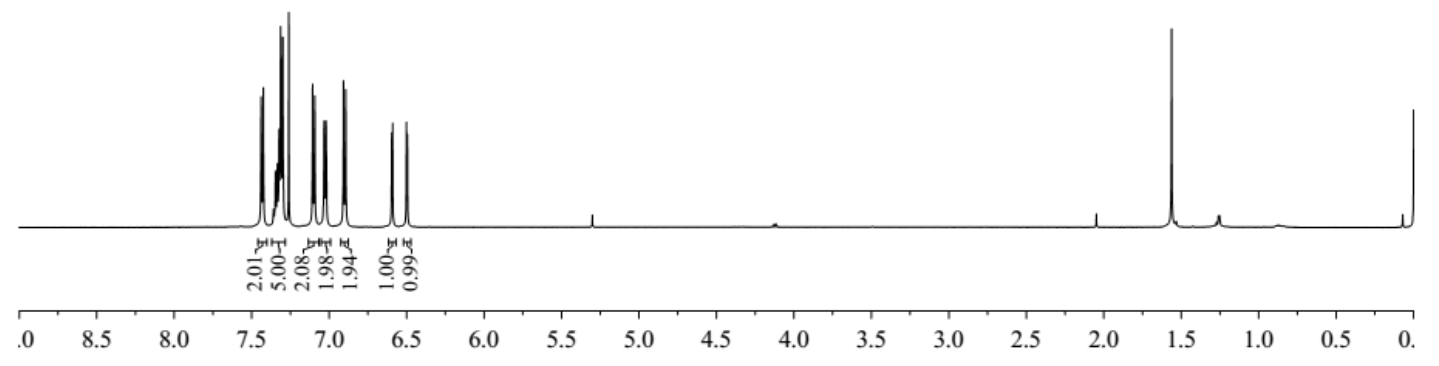

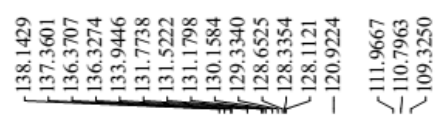

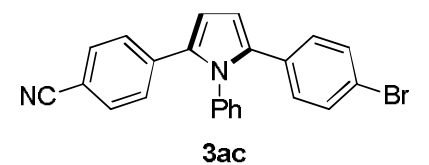

${ }^{13} \mathrm{C}$ NMR $\left(151 \mathrm{MHz}, \mathrm{CDCl}_{3}\right)$

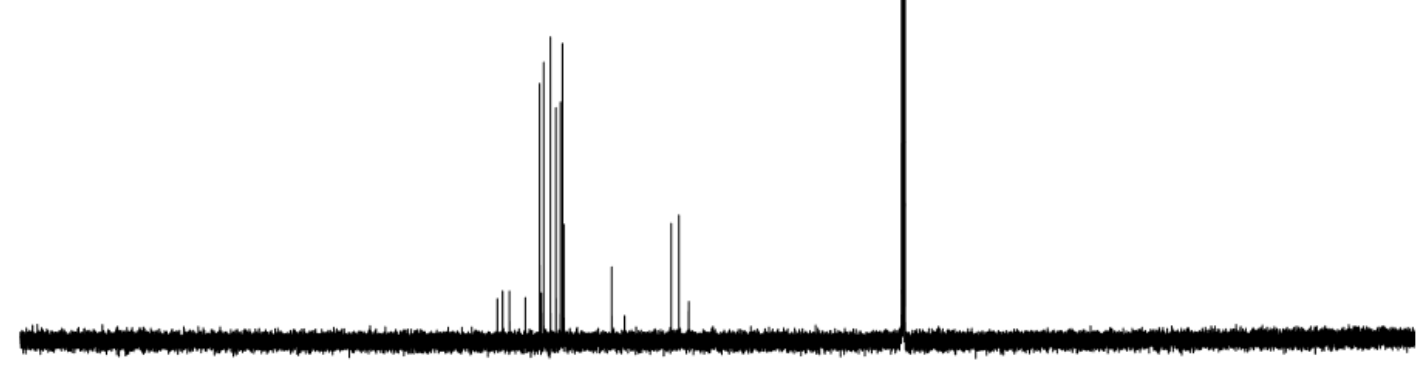

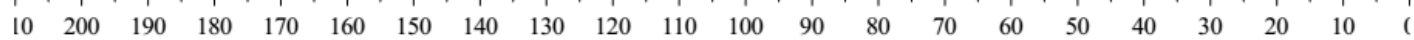




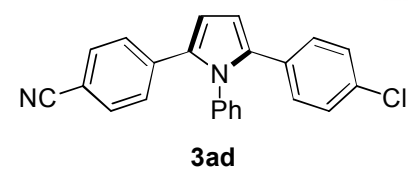

${ }^{1} \mathrm{H}$ NMR $\left(600 \mathrm{MHz}, \mathrm{CDCl}_{3}\right)$
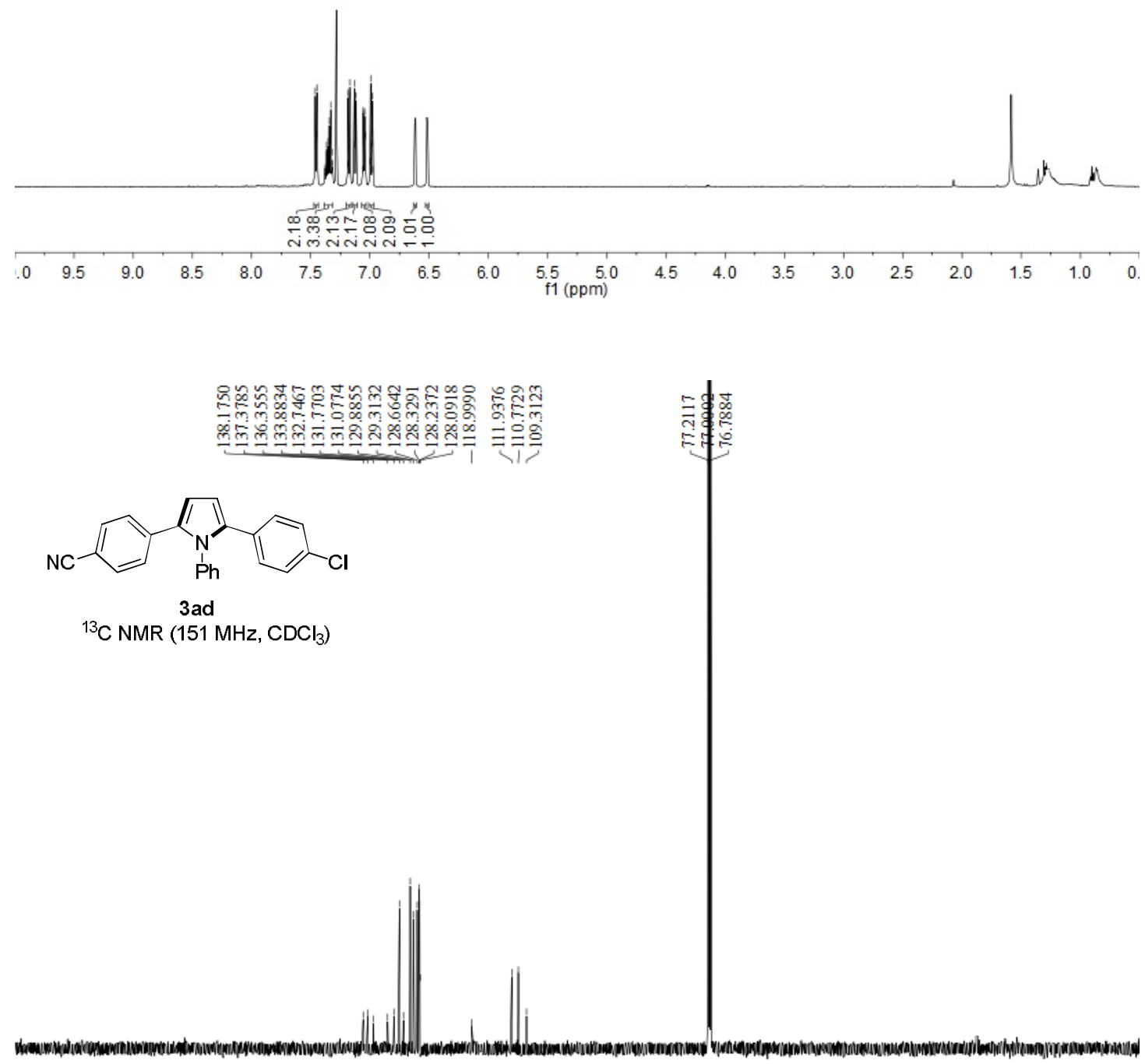

$\begin{array}{llllllllllllllllll}190 & 180 & 170 & 160 & 150 & 140 & 130 & 120 & 110 \underset{f}{100}(\mathrm{ppm}) & 90 & 80 & 70 & 60 & 50 & 40 & 30 & 20 & 10\end{array}$ 

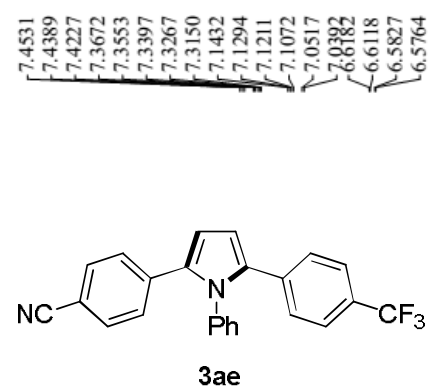

${ }^{1} \mathrm{H}$ NMR $\left(600 \mathrm{MHz}, \mathrm{CDCl}_{3}\right)$

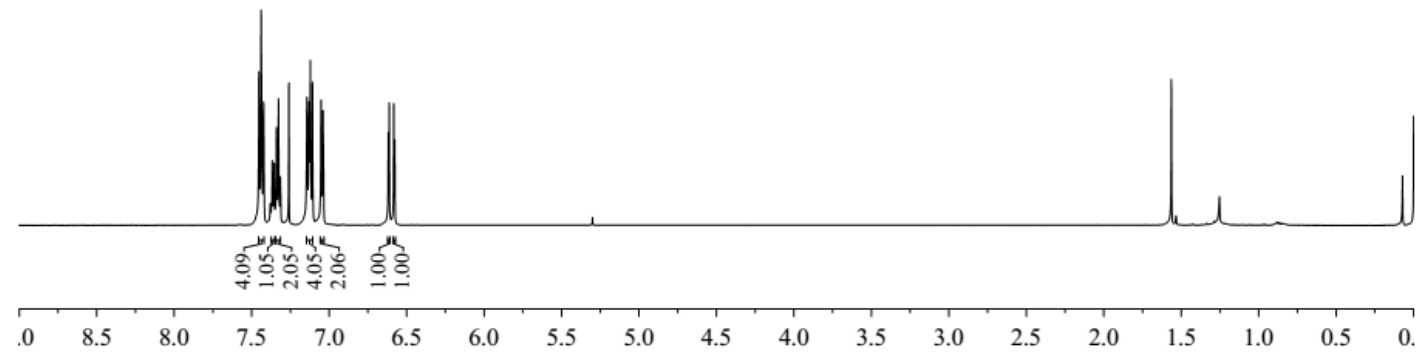

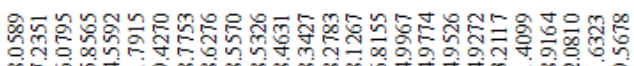

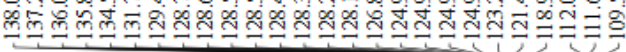

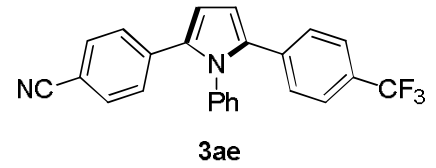

${ }^{13} \mathrm{C}$ NMR (151 MHz, $\mathrm{CDCl}$ )

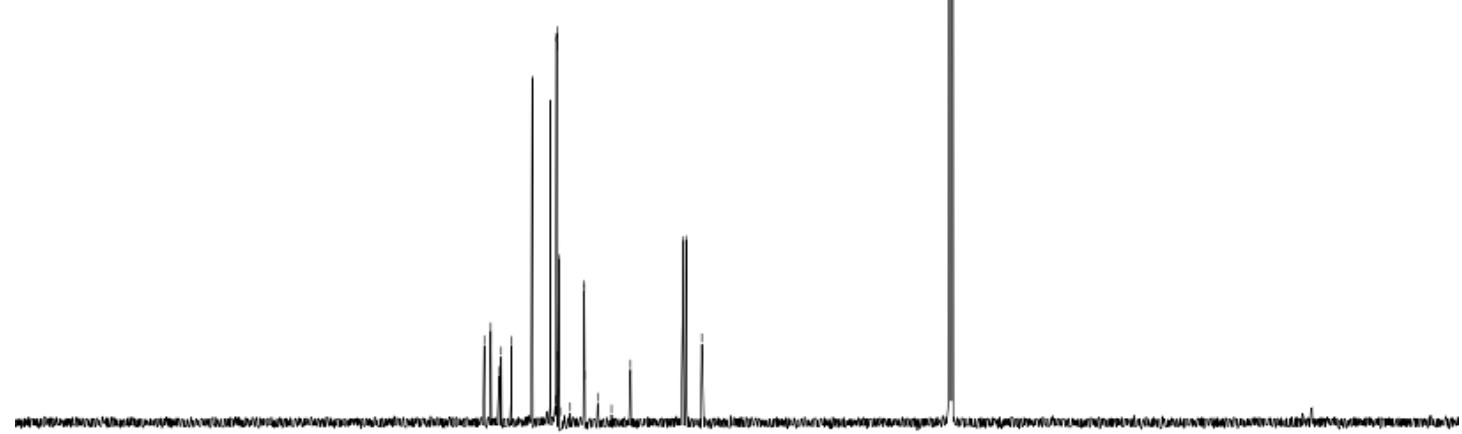

$\begin{array}{llllllllll}190 & 180 & 170 & 160 & 150 & 140 & 130 & 120 & \begin{array}{c}110 \\ \mathrm{f} 1(\mathrm{ppm})\end{array}\end{array}$

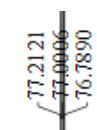




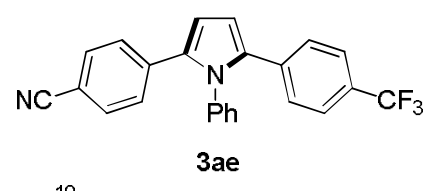

${ }^{19} \mathrm{~F}$ NMR $\left(565 \mathrm{MHz}, \mathrm{CDCl}_{3}\right)$
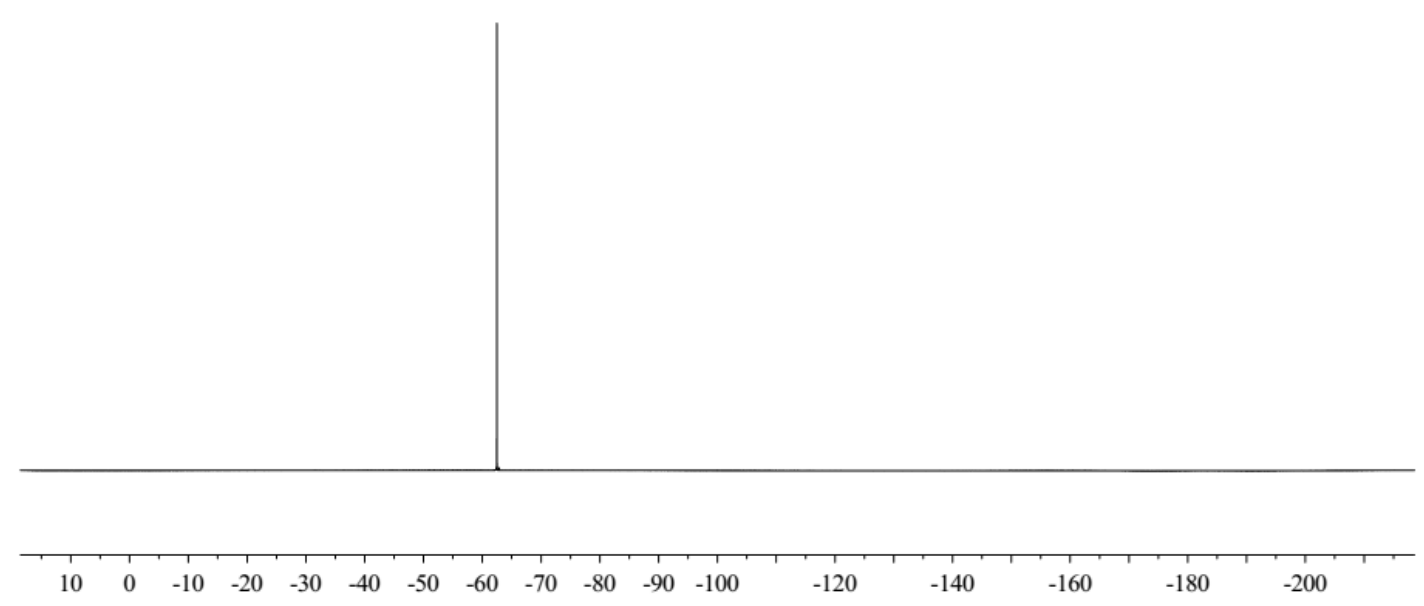

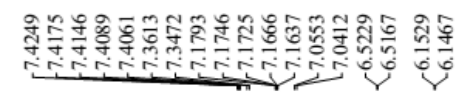

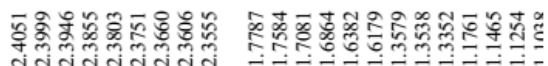

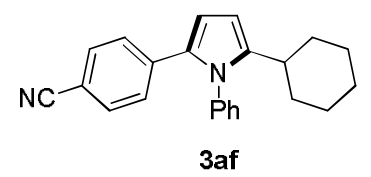

${ }^{1} \mathrm{H}$ NMR $\left(600 \mathrm{MHz}, \mathrm{CDCl}_{3}\right)$

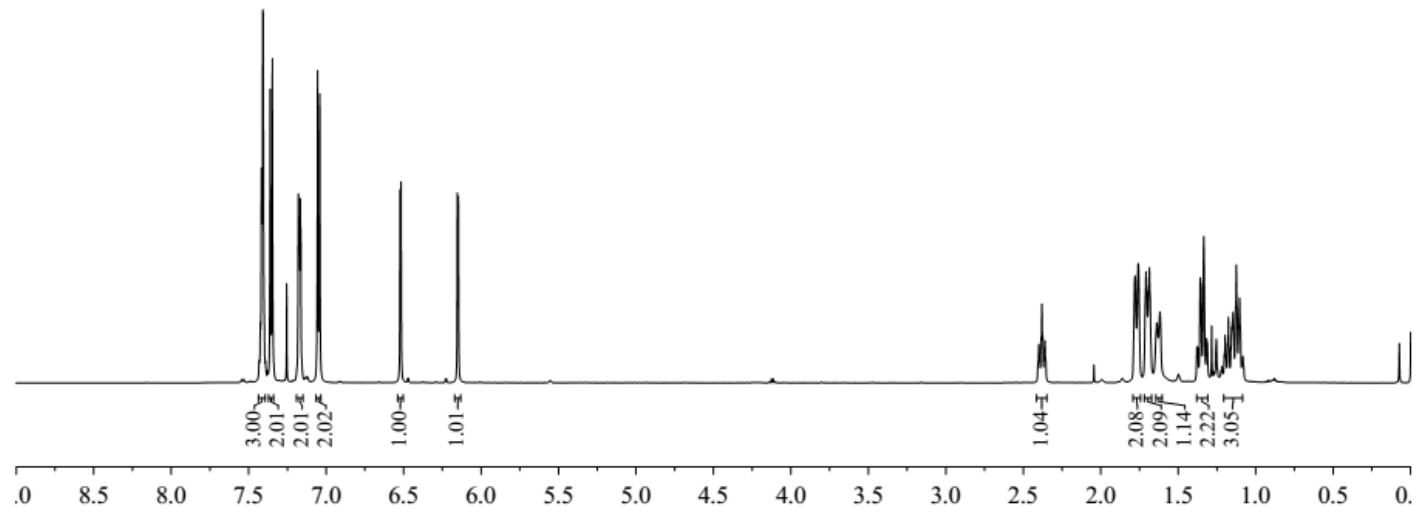




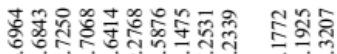

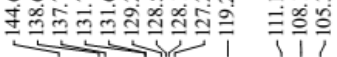

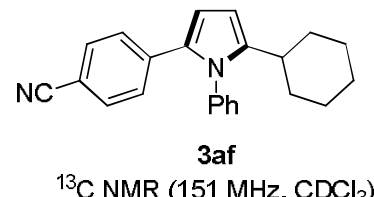

${ }^{13} \mathrm{C}$ NMR $\left(151 \mathrm{MHz}, \mathrm{CDCl}_{3}\right)$
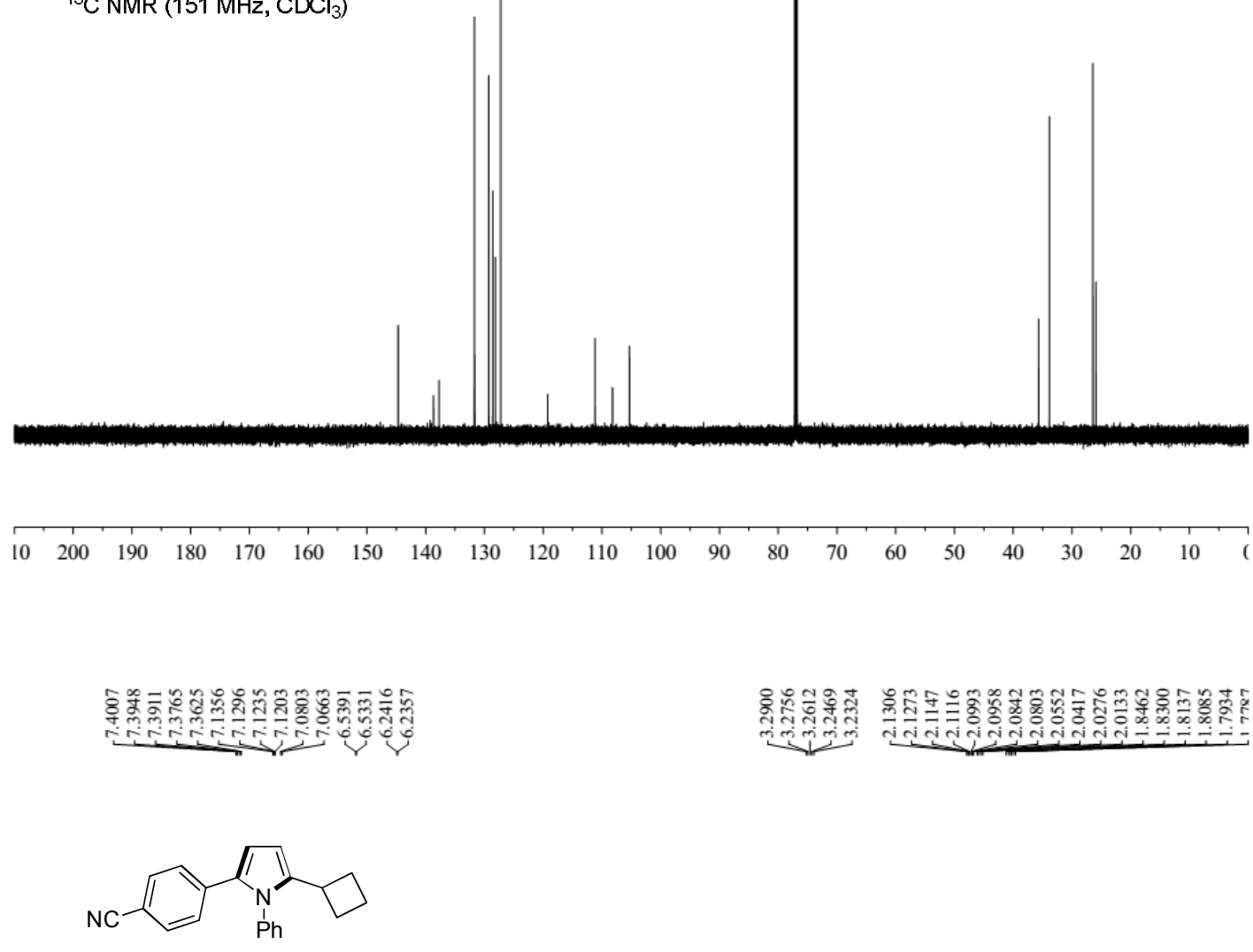

3ag

${ }^{1} \mathrm{H}$ NMR $\left(600 \mathrm{MHz}, \mathrm{CDCl}_{3}\right)$

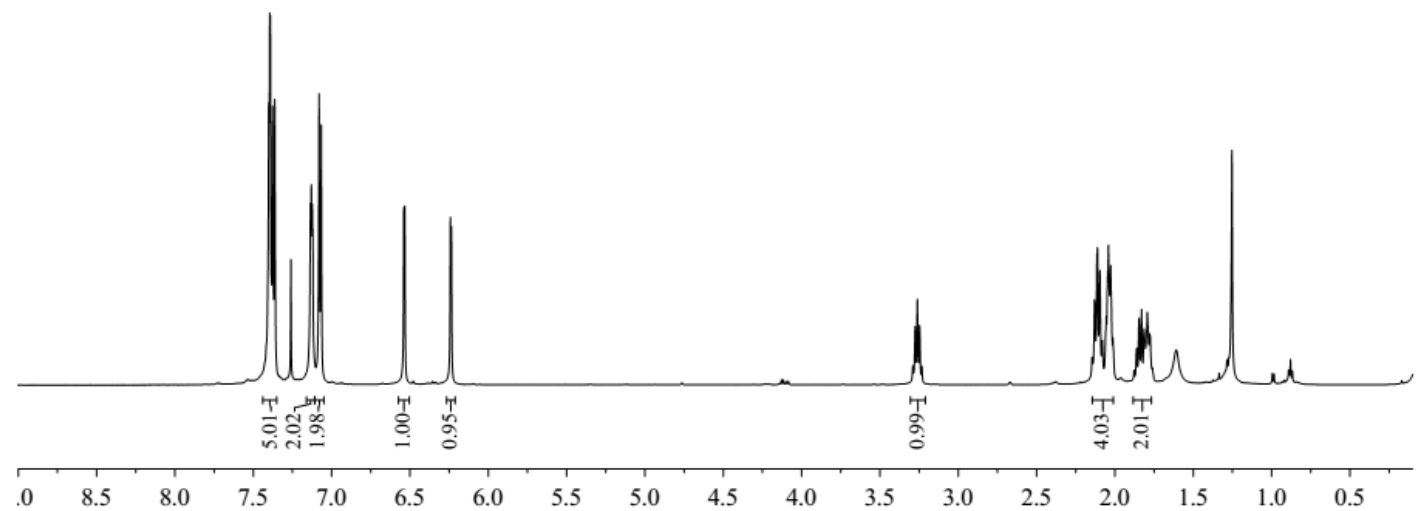




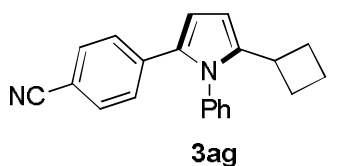

${ }^{13} \mathrm{C}$ NMR $\left(151 \mathrm{MHz}, \mathrm{CDCl}_{3}\right)$
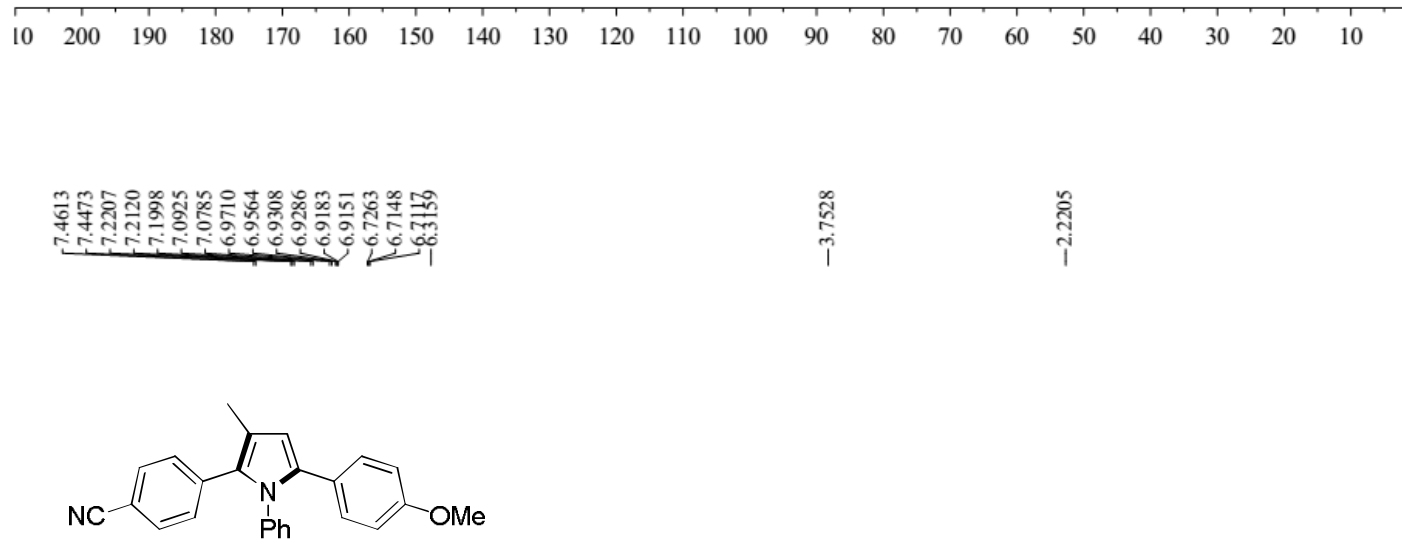

3ah

${ }^{1} \mathrm{H}$ NMR (600 MHz, $\mathrm{CDCl}_{3}$ )

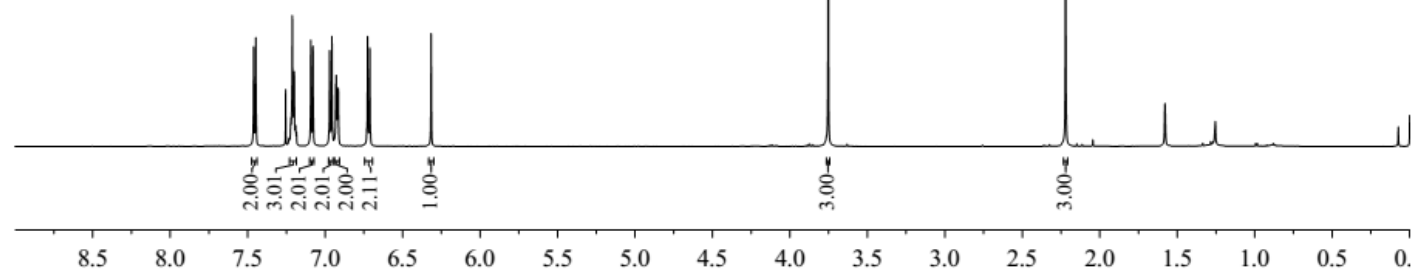



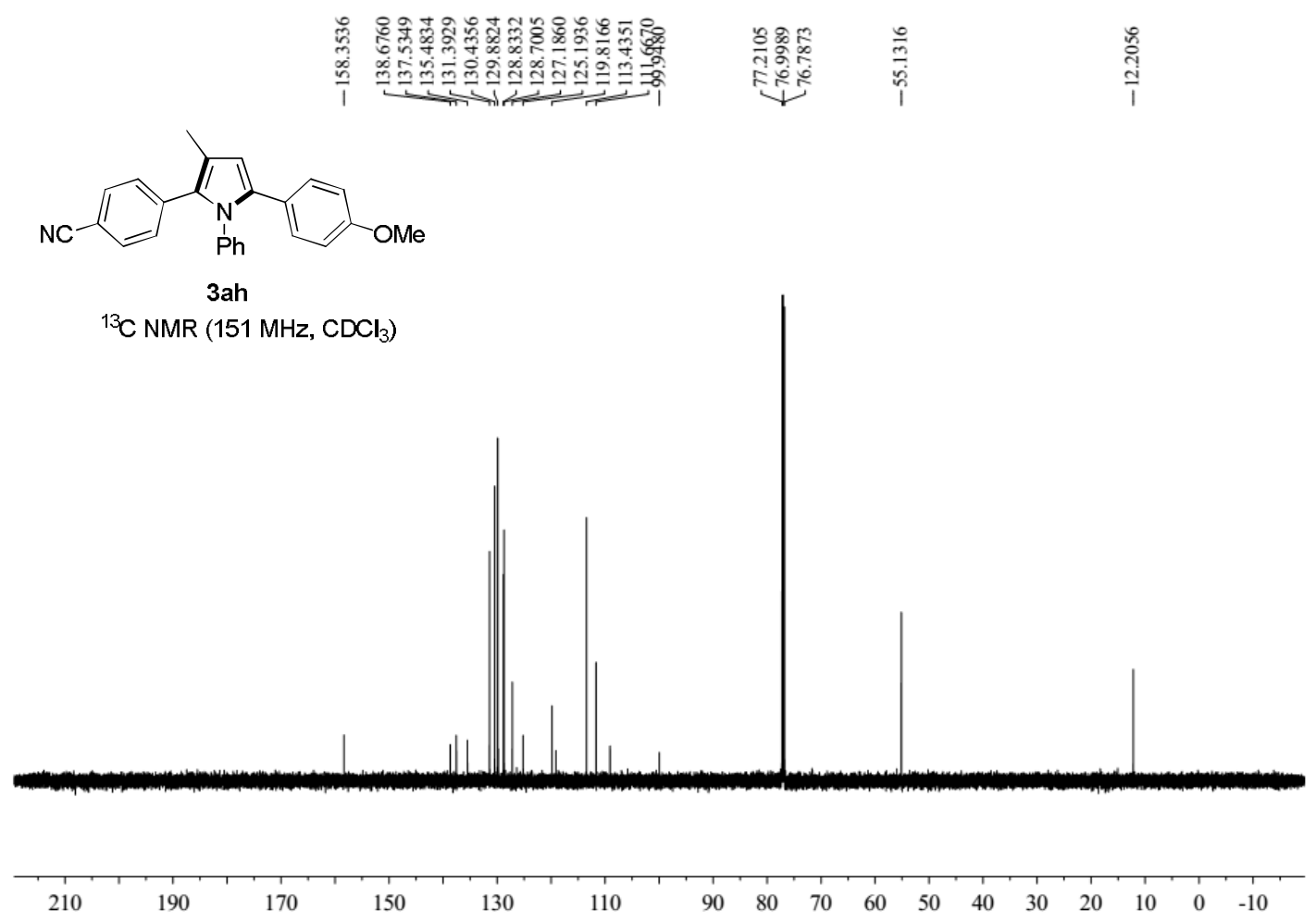

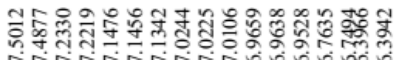 \\ 证}

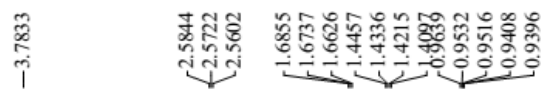<smiles>CCCCC(NCc1ccc(OC)cc1)c1ccc(C)cc1</smiles>

${ }^{1} \mathrm{H} \mathrm{NMR}\left(600 \mathrm{MHz}, \mathrm{CDCl}_{3}\right)$

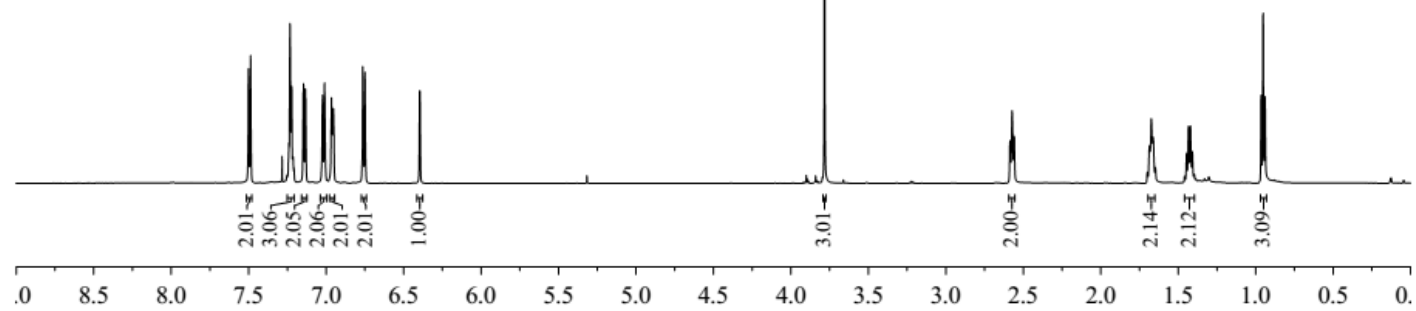




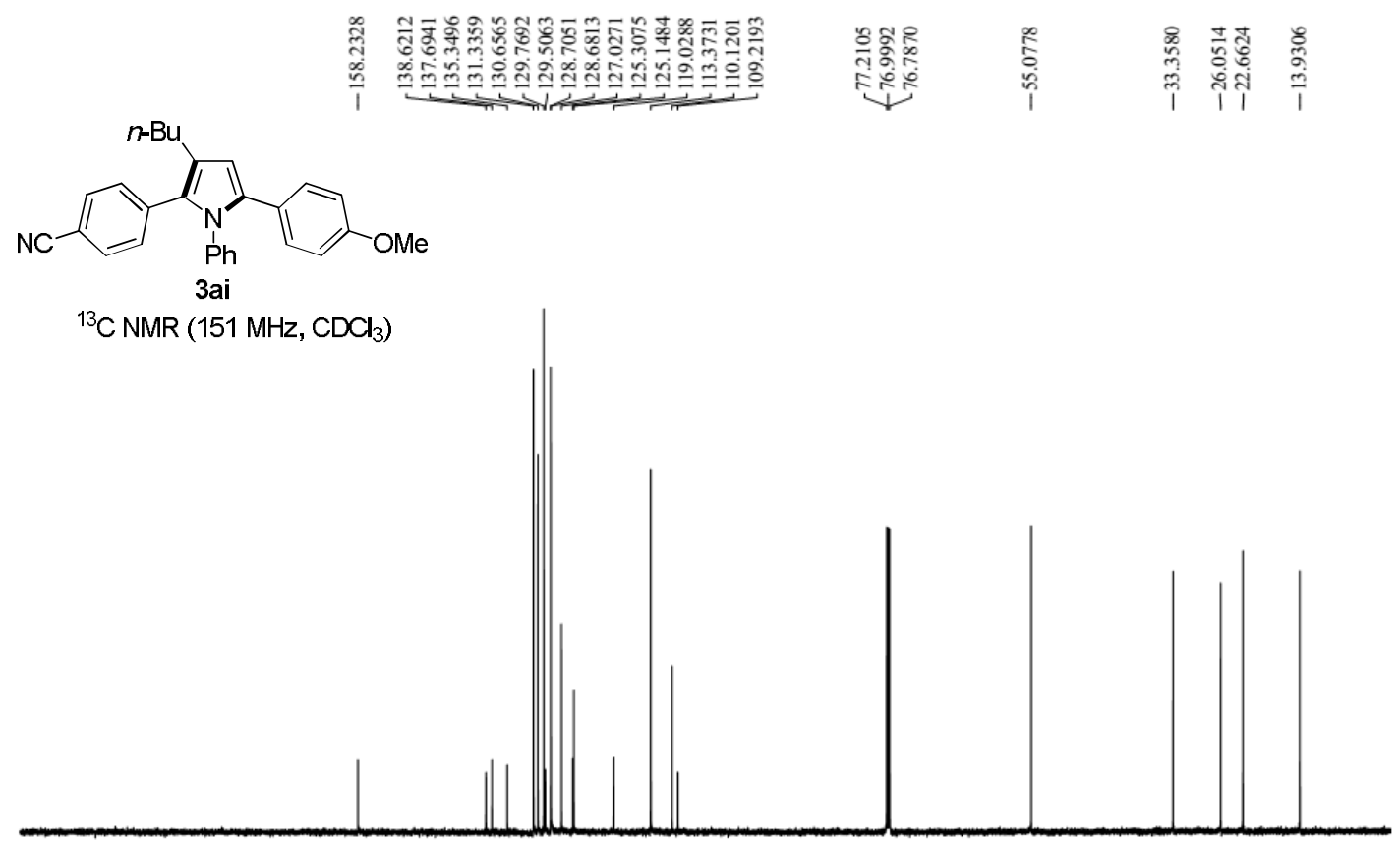

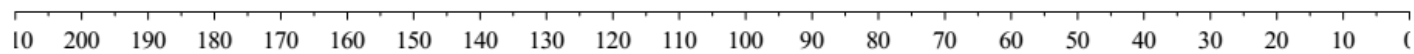

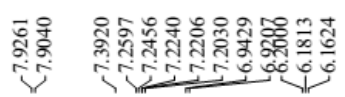

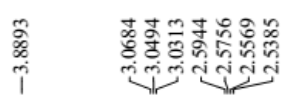

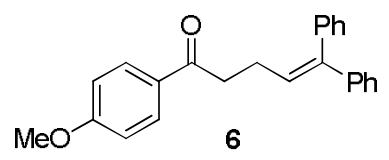

${ }^{1} \mathrm{H}$ NMR $\left(400 \mathrm{MHz}, \mathrm{CDCl}_{3}\right)$

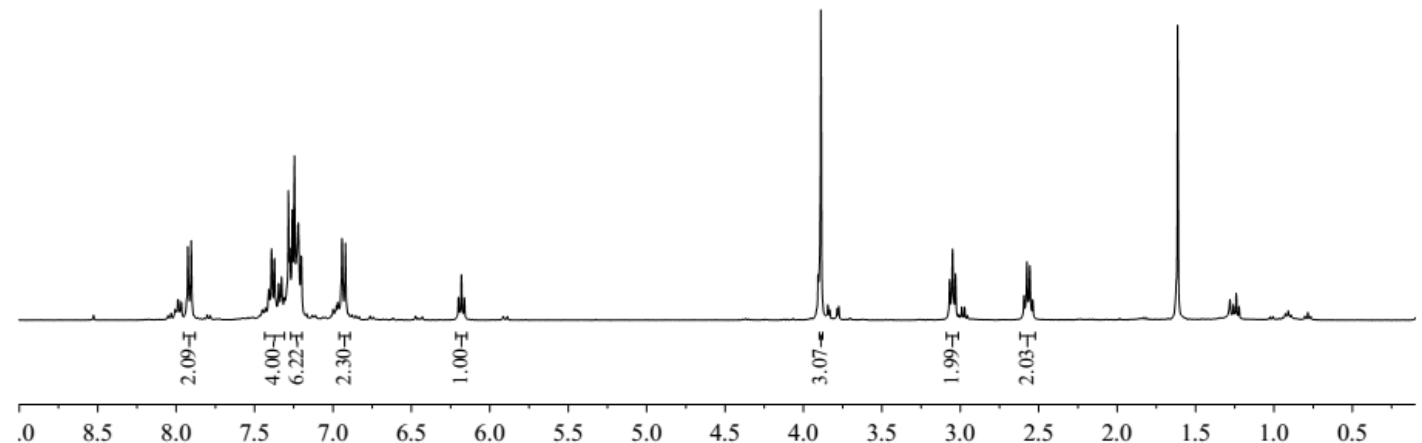




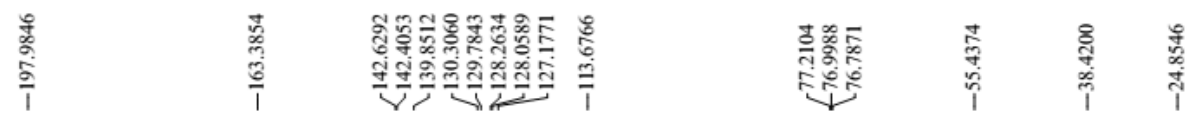<smiles>COc1ccc(C(=O)CCC=C(c2ccccc2)c2ccccc2)cc1</smiles>
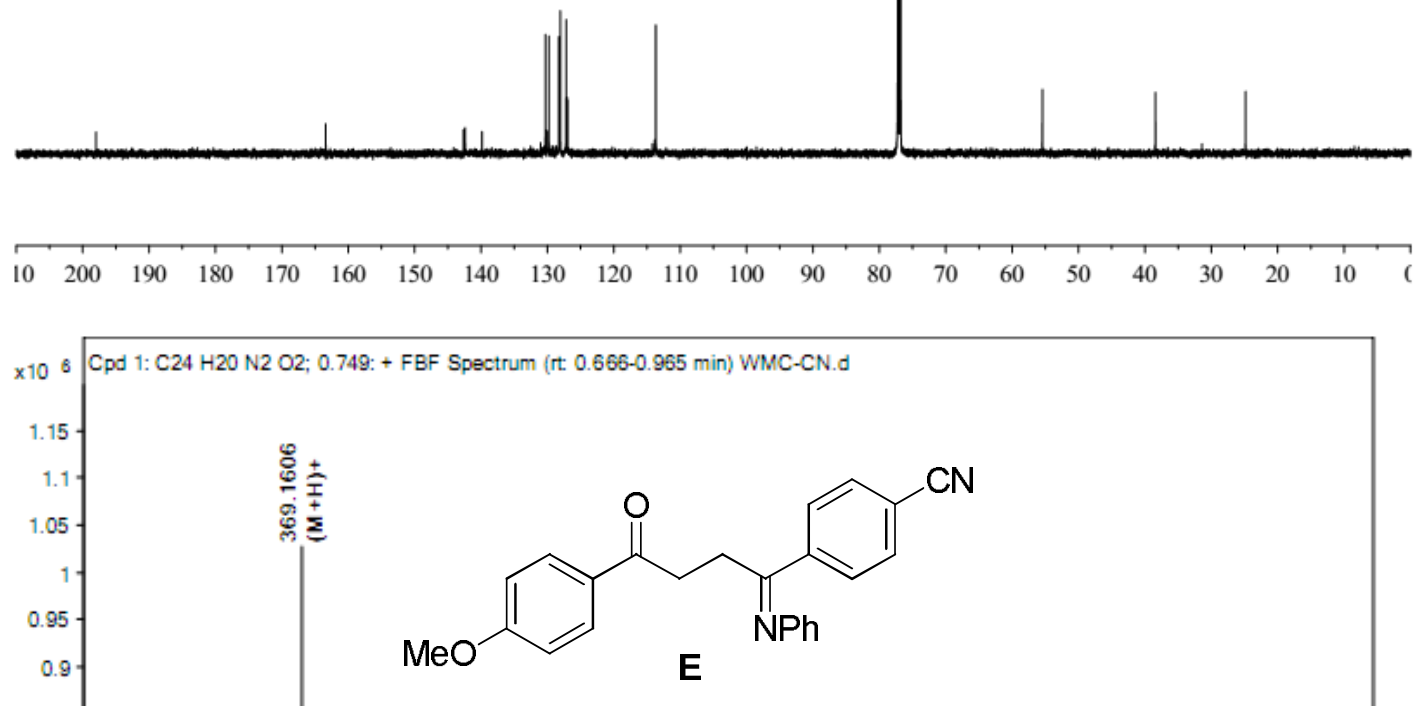

HRMS (ESI) $m / z:\left[M+\mathrm{H}^{+}\right.$Calcd for $\mathrm{C}_{24} \mathrm{H}_{21} \mathrm{~N}_{2} \mathrm{O}_{2}$ 369.1598; Found 369.1606.

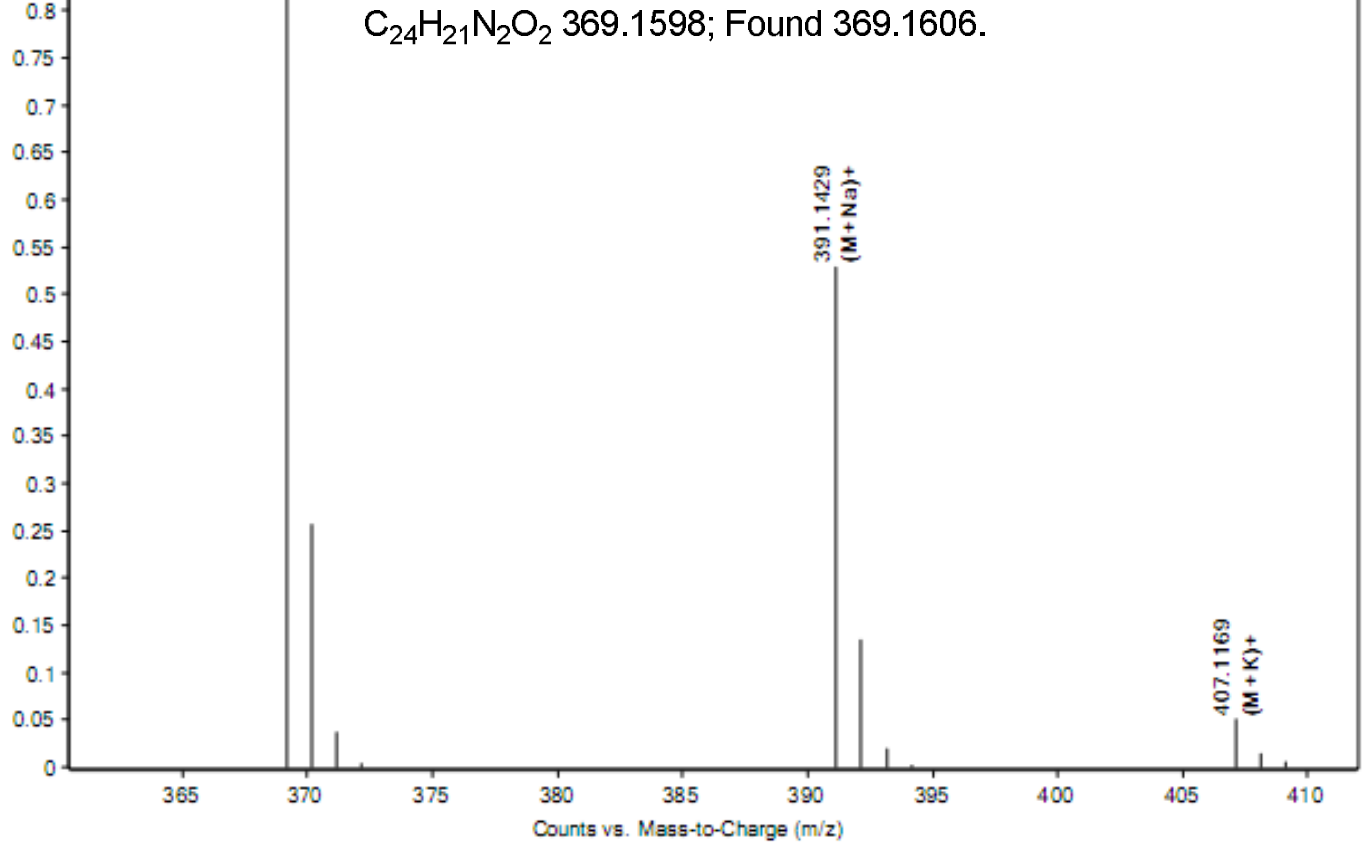

